\begin{tabular}{|c|l|}
\hline Title & Center Manifold Theory for the Motions of Camphor Boats with Delta Function \\
\hline Author(s) & Ikeda, Kota; Ei, Shin-Ichiro \\
\hline Citation & $\begin{array}{l}\text { Journal of dynamics and differential equations, 33(2), 621-657 } \\
\text { https://doi.org/10.1007/310884-020-09824-9 }\end{array}$ \\
\hline Issue Date & 2021-01-29 \\
\hline Doc URL & http://hdl.handle.net/2115/80313 \\
\hline Rights & $\begin{array}{l}\text { This is a post-peer-review, pre-copyedit version of an article published in Journal of dynamics and differential } \\
\text { equations. The final authenticated version is available online at: http:/dx.doi.org/10.1007/s10884-020-09824-9. }\end{array}$ \\
\hline Type & article (author version) \\
\hline File Information & Journal of Dynamics and Differential Equations 2020 29 Jan.pdf \\
\hline
\end{tabular}

Instructions for use 


\title{
Center manifold theory for the motions of camphor boats with delta function
}

\author{
Kota Ikeda · Shin-Ichiro Ei
}

Received: date / Accepted: date

\begin{abstract}
Various collective motions of camphor boats have been studied. Camphor boats are self-driven particles that interact with each other through the change of surface tension on water surface by camphor molecules. Consequently, even in a one-dimensional circuit, a congested state or jamming can be observed [20]. In this phenomenon, the unidirectional motion of each particle is considered a traveling wave, and the concentration of camphor molecules forms a pulse shape. Hence, each pair of particles interacts with each other like a pulse-pulse interaction. Thus, we expect that the center manifold theories proposed in [3] and [7] are applicable for the analysis of the collective motion of camphor boats. However, spatial discontinuity in our model, in particular the existence of Dirac delta functions in a linearized operator, does not fulfill the requirement in the reduction process because the authors developed their theories in $L^{2}$-framework and for smooth nonlinearity in [3] and [7]. In this article, we extend the results obtained in [3] and [7] and establish a new center manifold approach in $\left(H^{1}\right)^{*}$-framework. Finally, we succeed to rigorously reduce a mathematical model ([17]) coupled with a Newtonian equation and a reaction-diffusion equation including delta functions to an ordinary differential system that represents the motions of camphor boats.
\end{abstract}

Keywords center manifold theory - bifurcation - traveling wave solution . collective motion

Mathematics Subject Classification (2010) 37L10 $\cdot 35 \mathrm{C} 07 \cdot 35 \mathrm{~K} 57$

\author{
K. Ikeda \\ Meiji University, 4-21-1, Nakano, Nakano-ku, Tokyo 164-8525 JAPAN \\ Tel.: +81-3-5343-8357 \\ E-mail: ikeda@meiji.ac.jp \\ S.-I. Ei \\ Hokkaido University, Sapporo 060-0810 JAPAN
}




\section{Introduction}

Self-driven motion of animal and inanimal organisms is observed in several fields, including biology [16], chemistry [2], and nonlinear physics [10], [21], [22]. Organisms move spontaneously to aggregate and form self-organized structures. In many cases, the individual members do not interact directly. Rather, they change their surroundings in ways that have an influence on the behavior of other members, which indicates that the organisms have long-range interactions [9], [14]. Therefore, it is important not only to clarify the mechanism of the self-sustaining motion of each organism but also to study how organisms behave as a whole system.

Spatiotemporal collective motions in chemical experiments with camphor have been investigated in [17], [18], [20]. A camphor scraping at an air-water surface exhibits several motions, including clockwise / counterclockwise rotation, and translation ([18]). Moreover, it was shown in [17] and [20] that a unidirectional motion can be observed if we place a camphor boat in an annular water channel. In an experimental setup, a camphor boat is composed of a plastic disk and a camphor disk stuck on the edge of the plastic disk with an adhesive. Camphor boats constitute a system for changing the number of particles with simple interaction. In this system, we observe two different states depending on the number of boats. It was reported in [20] that, when the number of camphor boats is less than 30, they move with a constant velocity and spatially disperse with the same spacing between the boats, which is called a homogeneous state. However, when the number of boats is greater than 30, the velocities of the boats change with temporal oscillation, and the shock wave appears in the line of the boats, which is an inhomogeneous state.

Various motions exhibited by a camphor boat have been studied mathematically. In this article, based on [17] and [20], we introduce the following mathematical model for the self-sustaining motion of a camphor boat:

$$
\left\{\begin{array}{l}
\frac{d^{2} x_{\infty}}{d t^{2}}=-\mu \frac{d x_{\infty}}{d t}+\gamma\left(u\left(x_{\infty}+\rho, t\right)\right)-\gamma\left(u\left(x_{\infty}-\rho, t\right)\right) \\
\frac{\partial u}{\partial t}=\frac{\partial^{2} u}{\partial x^{2}}-\alpha u+f\left(x-x_{\infty}, s\right)
\end{array}\right.
$$

where $\alpha, \mu$, and $\rho$ are positive constants. The first equation is a Newtonian equation of motion for the camphor boat with the surface tension of water given by $\gamma(u)$ as a smooth and monotonically decreasing function of $u$. In this model, a camphor scraping is regarded as a particle, and the position of a camphor boat is denoted by $x_{\infty}=x_{\infty}(t)$. The surface concentration, denoted by $u$, of a camphor molecular layer is assumed to obey the reaction-diffusion equation with the function $f(x, s)$ defined by

$$
f(x, s) \equiv \begin{cases}1, & 0<x<\rho \\ s, & -\rho<x<0 \\ 0, & \text { otherwise }\end{cases}
$$

which indicates that camphor molecules are supplied only from $(-\rho, \rho)$ where a camphor boat contacts the water surface. Let $s \in[0,1]$, which indicates that a camphor boat considered in this model is an inhomogeneous object and the amount of supply on $(-\rho, 0)$ is less than or equal to that on $(0, \rho)$. This parameter $s$ does not appear in models proposed previously in [17] and [20], but was introduced in 
the previous works [4], [5]. It provides a new type of a bifurcation structure in (1), which will be described later.

The spontaneous motion of a camphor boat can be characterized by a traveling wave solution in (1), which is a stationary solution of

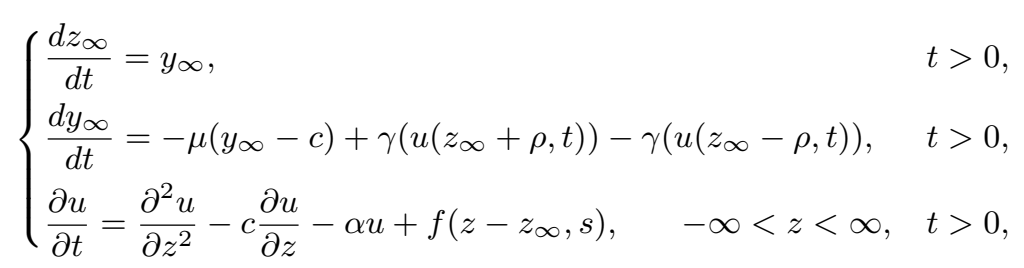

where we set $z_{\infty}=z_{\infty}(t) \equiv x_{\infty}(t)+c t, y_{\infty}=y_{\infty}(t) \equiv\left(d z_{\infty} / d t\right)(t)$, and $z \equiv$ $x+c t$ in (1). We represent the right-hand side of $(2)$ by $\mathcal{L}_{\infty}\left(z_{\infty}, y_{\infty}, u ; c, \mu, s\right)$ for ${ }^{t}\left(z_{\infty}, y_{\infty}, u\right) \in \mathbb{R}^{2} \times H^{1}(\mathbb{R})$, where ${ }^{t}$ denotes the transpose and $H^{k}(\mathbb{R})(k=1,2)$ is a usual Sobolev space. We note that $\mathcal{L}_{\infty}\left(z_{\infty}, y_{\infty}, u ; c, \mu, s\right)$ is considered an operator from $\mathbb{R}^{2} \times H^{1}(\mathbb{R})$ to $\mathbb{R}^{2} \times\left(H^{1}(\mathbb{R})\right)^{*}$, where $\left(H^{1}(\mathbb{R})\right)^{*}$ denotes the dual space of $H^{1}(\mathbb{R})$. A stationary solution defined by $\left(z_{\infty}, y_{\infty}, u\right)=(0,0, p)$ of $(2)$ for a function $p=p(z)$ is called a traveling wave solution, and the parameter $c$ is called wave speed. The function $p$ in traveling wave solutions of (2) is considered to be in $H^{2}(\mathbb{R})$.

As shown in [17], there is a critical value of $\mu$ such that (2) for $s=1$ has a stable traveling wave solution with a positive wave speed only in the case that $\mu$ is smaller than the critical value. Hence, the pair of the critical value and $c=0$ generates a supercritical pitchfork bifurcation, in which the spatially symmetric structure is crucial. Moreover, the authors in [17] states the existence of a saddlenode bifurcation. There is no Hopf bifurcation point in (2) as far as we investigated numerically.

Such a bifurcation structure for $s=1$ is preserved in the case of $s<1$. A saddlenode bifurcation point is easily found while the existence of a pitchfork bifurcation point is not trivial because the assumption of $s<1$ leads to an asymmetric structure in the system and probably causes an imperfect bifurcation. Nevertheless, a pitchfork bifurcation can occur in (2) for some $s<1$. For example, there is a bifurcation point $\left(\mu_{0}, s_{0}, c_{0}\right)$ such that $0.298<\mu_{0}<0.3,0.12<s_{0}<0.13$, and $0.271<c_{0}<0.273$ for $\gamma(u)=\gamma_{1} /(1+a u)$ and $\left(a, \gamma_{1}, \alpha, \rho\right)=(0.64,1.7,0.011,0.84)$. From this viewpoint, both $\mu$ and $s$ should change simultaneously in order that a pitchfork bifurcation in (2) appears. Under a more general setting in the nonlinearity $\gamma(u)$, we do not know whether the system (2) exhibits such a bifurcation structure.

Let $\left(\mu_{0}, s_{0}, c_{0}\right)$ be a bifurcation point of a traveling wave solution $(0,0, p)$ with a wave speed $c_{0}$. We will give the precise description of a bifurcation point in the assumptions (A1)-(A3). The existence of such a bifurcation point affects the linearized operator

$$
\begin{aligned}
& \mathcal{L}_{\infty}^{\prime}\left(0,0, p ; c_{0}, \mu_{0}, s_{0}\right) \Phi \\
& \equiv\left(\begin{array}{c}
Y_{\Phi} \\
-\mu_{0} Y_{\Phi}+\gamma^{\prime}(p(\rho))\left(p^{\prime}(\rho) Z_{\Phi}+\phi(\rho)\right)-\gamma^{\prime}(p(-\rho))\left(p^{\prime}(-\rho) Z_{\Phi}+\phi(-\rho)\right) \\
\frac{\partial^{2} \phi}{\partial z^{2}}-c \frac{\partial \phi}{\partial z}-\alpha \phi-Z_{\Phi}\left[s_{0} \delta_{-\rho}-\left(s_{0}-1\right) \delta_{0}-\delta_{\rho}\right]
\end{array}\right)
\end{aligned}
$$


for $\Phi={ }^{t}\left(Z_{\Phi}, Y_{\Phi}, \phi\right) \in \mathbb{R}^{2} \times H^{1}(\mathbb{R})$, where we denote the Dirac delta function providing unit mass to the point $z_{0}$ by $\delta_{z_{0}}$. Throughout this article, we regard $\mathcal{L}_{\infty}^{\prime}\left(0,0, p ; c_{0}, \mu_{0}, s_{0}\right)$ as a closed operator from $\mathbb{R}^{2} \times H^{1}(\mathbb{R})$ to $\mathbb{R}^{2} \times\left(H^{1}(\mathbb{R})\right)^{*}$. It is evident that $\Phi \equiv{ }^{t}(-1,0, \phi)$ satisfies $\mathcal{L}_{\infty}^{\prime}\left(0,0, p ; c_{0}, \mu_{0}, s_{0}\right) \Phi=0$, i.e., $\Phi$ is an eigenfunction of $\mathcal{L}_{\infty}^{\prime}\left(0,0, p ; c_{0}, \mu_{0}, s_{0}\right)$ for the zero eigenvalue, where $\phi$ can be defined by $\phi \equiv p^{\prime}$.

Furthermore, (3) has a degeneracy condition, and there is a solution $\Psi \equiv$ ${ }^{t}(0,1, \psi)$ of $\mathcal{L}_{\infty}^{\prime}\left(0,0, p ; c_{0}, \mu_{0}, s_{0}\right) \Psi=-\Phi$. Here, we assume that the first component of $\Psi$ is 0 without loss of generality. From this condition, we note that $\psi \in H^{2}(\mathbb{R})$ as shown below. The existence of $\Psi$ indicates that the multiplicity of the zero eigenvalue of $\mathcal{L}_{\infty}^{\prime}\left(0,0, p ; c_{0}, \mu_{0}, s_{0}\right)$ is equal to or more than 2.

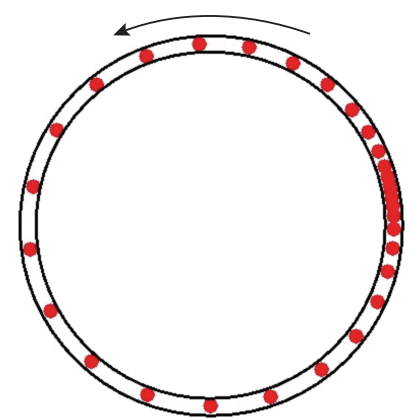

Fig. 1 Congestion in (4). The red disks represent camphor boats. All camphor boats move in the same direction as indicated by the arrow.

Thus, we assume throughout this article that there is $\left(\mu_{0}, s_{0}, c_{0}\right)$ such that the following conditions hold true:

(A1) $\mathcal{L}_{\infty}\left(0,0, p ; c_{0}, \mu_{0}, s_{0}\right)=0$ has a solution $p=p(z) \in H^{2}(\mathbb{R})$.

(A2) There is a solution $\Psi \in \mathbb{R}^{2} \times H^{2}(\mathbb{R})$ of $\mathcal{L}_{\infty}^{\prime}\left(0,0, p ; c_{0}, \mu_{0}, s_{0}\right) \Psi=-\Phi \equiv$ ${ }^{t}(-1,0, \phi)$, where $\phi \equiv p^{\prime}$. In addition, the generalized eigenspace associated to a zero eigenvalue is spanned by $\Phi$ and $\Psi$.

(A3) Any spectrum $\lambda$ of $\mathcal{L}_{\infty}^{\prime}\left(0,0, p ; c_{0}, \mu_{0}, s_{0}\right)$ is contained in $\mathbb{C} \backslash \Sigma$ except for $\lambda=0$, where $\Sigma \equiv\{\lambda \in \mathbb{C} \mid-\alpha / 2 \leq \operatorname{Re} \lambda\} \cup\{\lambda \in \mathbb{C}|| \arg \lambda \mid \leq \theta\}$ for some $\theta>\pi / 2$. Re $\lambda$ and $\arg \lambda$ denote the real part and argument of a complex value $\lambda$, respectively, where $\arg \lambda$ is assumed to satisfy $-\pi<\arg \lambda \leq \pi$.

Here, we provide a few remarks on the regularity and uniqueness of $\Psi$. First, we check that the first component of $\Psi$ is 0 under the assumption (A2). If the first component of $\Psi$ is not equal to 0 , the third component of $\mathcal{L}_{\infty}^{\prime}\left(0,0, p ; c_{0}, \mu_{0}, s_{0}\right) \Psi$ has the three delta functions. Thus, we observe that $\Psi \notin \mathbb{R}^{2} \times H^{2}(\mathbb{R})$. However, if the first component of $\Psi$ is equal to 0 , then $\Psi \in \mathbb{R}^{2} \times H^{2}(\mathbb{R})$ because of $\Phi \in$ $\mathbb{R}^{2} \times H^{1}(\mathbb{R})$. The regularity of $\Psi$ is important for technical reasons. 
Now we consider the collective motion of $(N+1)$-camphor boats in a onedimensional circuit of length $L$. Our system is described by

$$
\left\{\begin{array}{l}
\frac{d z_{i}}{d t}=y_{i} \\
\frac{d y_{i}}{d t}=-\left(\mu_{0}+\kappa_{1}\right)\left(y_{i}-c_{0}\right)+\gamma\left(u\left(z_{i}+\rho, t\right)\right)-\gamma\left(u\left(z_{i}-\rho, t\right)\right) \\
\frac{\partial u}{\partial t}=\frac{\partial^{2} u}{\partial z^{2}}-c_{0} \frac{\partial u}{\partial z}-\alpha u+\sum_{i=0}^{N} \bar{f}\left(z-z_{i}, s_{0}+\kappa_{2}\right)
\end{array}\right.
$$

in $z \in \mathbb{T} \equiv \mathbb{R} / L \mathbb{Z}$ for $N \geq 0$ and $i=0, \ldots, N$, where $z_{i}=z_{i}(t), y_{i}=y_{i}(t)$, and $u=u(z, t) \in H^{1}(\mathbb{T})$. Here, we denote by $H^{k}(\mathbb{T})(k=1,2)$ a functional space defined by the closure of $C^{\infty}(\mathbb{T})$ in $H^{k}(0, L)$, where $C^{\infty}(\mathbb{T})$ is a set of infinitely differentiable and $L$-periodic functions $\varphi: \mathbb{R} \rightarrow \mathbb{R}$. For simplicity, we denote an operator associated with the right-hand sides of the first, second, and third equations of (4) by $\mathcal{L}(U, \kappa) \equiv{ }^{t}\left(\mathcal{L}_{0}^{z}(U), \mathcal{L}_{0}^{y}\left(U, \kappa_{1}\right), \ldots, \mathcal{L}_{N}^{z}(U), \mathcal{L}_{N}^{y}\left(U, \kappa_{1}\right), \mathcal{L}^{u}\left(U, \kappa_{2}\right)\right)$, where $U={ }^{t}\left(z_{0}, y_{0}, \ldots, z_{N}, y_{N}, u\right) \in X^{1} \equiv \mathbb{R}^{2 N+2} \times H^{1}(\mathbb{T}), \kappa=\left(\kappa_{1}, \kappa_{2}\right) \equiv\left(\mu-\mu_{0}, s-s_{0}\right)$, and $\mathcal{L}_{i}^{z}, \mathcal{L}_{i}^{y}(i=0, \ldots, N), \mathcal{L}^{u}$ are given by

$$
\begin{aligned}
\mathcal{L}_{i}^{z}(U) & \equiv y_{i} \\
\mathcal{L}_{i}^{y}\left(U, \kappa_{1}\right) & \equiv-\left(\mu_{0}+\kappa_{1}\right)\left(y_{i}-c_{0}\right)+\gamma\left(u\left(z_{i}+\rho\right)\right)-\gamma\left(u\left(z_{i}-\rho\right)\right), \\
\mathcal{L}^{u}\left(U, \kappa_{2}\right) & \equiv \frac{\partial^{2} u}{\partial z^{2}}-c_{0} \frac{\partial u}{\partial z}-\alpha u+\sum_{i=0}^{N} \bar{f}\left(z-z_{i}, s_{0}+\kappa_{2}\right) .
\end{aligned}
$$

We note that $\mathcal{L}(U, \kappa)$ is considered an operator from $X^{1}$ to $X \equiv \mathbb{R}^{2 N+2} \times\left(H^{1}(\mathbb{T})\right)^{*}$ as well as $\mathcal{L}_{\infty}\left(z_{\infty}, y_{\infty}, u ; c, \mu, s\right)$, where $\left(H^{1}(\mathbb{T})\right)^{*}$ is the dual space of $H^{1}(\mathbb{T})$. For a function $\varphi$ defined on $(-\infty, \infty), \bar{\varphi}$ denotes an $L$-periodic function given by

$$
\bar{\varphi}(z) \equiv \chi(z-n L) \varphi(z-n L), \quad\left(n-\frac{1}{2}\right) L<z<\left(n+\frac{1}{2}\right) L, \quad n \in \mathbb{Z},
$$

where $\chi \in C^{\infty}(\mathbb{R})$ is a cut-off function defined by

$$
\chi(z) \equiv \begin{cases}1, & |z|<\frac{L}{4} \\ 0, & |z|>\frac{3 L}{8} .\end{cases}
$$

Let $l$ be the position of the 0th particle and let us denote the distance between the $i$-th and the $(i+1)$-th particles by $h_{i}$ for $i=0, \ldots, N-1$. As we consider a one-dimensional circuit, $h_{N}$ is defined by the distance between the $N$-th and the 0th particles, i.e., $h_{N}=L-\sum_{i=0}^{N-1} h_{i}$. Thus, we define a relative position of the $i$-th particle by $\bar{z}_{i}=\bar{z}_{i}(\mathbf{h}) \equiv \sum_{j=0}^{i \bar{i}-1} h_{j}$, where $\mathbf{h} \equiv{ }^{t}\left(h_{0}, \ldots, h_{N-1}\right)$. We consider $\bar{z}_{0} \equiv 0$. Here, we assume that there is a sufficient distance from one particle to any other particle. In other words, $h_{i}$ is assumed to satisfy $h_{i}>h^{*}$ for any $i=0, \ldots, N$, where $h^{*}>0$ is assumed to be sufficiently large. From this assumption, the circuit length $L$ is also assumed to be large, depending on $h^{*}$. More precisely, we give $N \geq 0$ and a positive constant $C_{*}$ arbitrarily and independently of $L$ and $h^{*}$, and suppose that $L$ and $h^{*}$ satisfy

$$
\frac{L}{N+1}-C_{*} \leq h^{*} \leq \frac{L}{N+1} .
$$


Throughout this article, $L$ and $h^{*}$ are assumed to be sufficiently large under the condition (6).

Owing to the interactions described by (4), the positions of the particles will be varied. Thus, the position $l$ and the distance $h_{i}$ are regarded as functions of time, denoted by $l(t)$ and $h_{i}(t)$, respectively. In the statement of our result, we use several notations. Consider $P(z, l, \mathbf{h}) \equiv \sum_{i=0}^{N}\left[\left(\bar{z}_{i}+l\right) e_{2 i+1}+\bar{p}\left(z-\bar{z}_{i}-l\right) e_{2 N+3}\right]$, $\xi(z, l, \mathbf{h}, \mathbf{r}) \equiv \sum_{i=0}^{N} r_{i}\left[e_{2 i+2}+\bar{\psi}\left(z-\bar{z}_{i}-l\right) e_{2 N+3}\right]$, and $S(z, l, \mathbf{h}, \mathbf{r}) \equiv P(z, l, \mathbf{h})+$ $\xi(z, l, \mathbf{h}, \mathbf{r})$, where $e_{i}$ is the unit vector in $\mathbb{R}^{2 N+3}$ given by $e_{i} \equiv{ }^{t}(0, \ldots, 0, \stackrel{i}{1}$ $, 0, \ldots, 0)$ and $\mathbf{r} \equiv{ }^{t}\left(r_{0}, \ldots, r_{N}\right)$. Note that $S(z, l, \mathbf{h}, \mathbf{r}) \in \mathbb{R}^{2 N+2} \times H^{2}(\mathbb{T})$.

In the same way as in (2), we can show that there exists a traveling wave solution of (4) such that $z_{i}=i L /(N+1)$, which corresponds to the homogeneous state observed in the experiment of [20]. According to the result obtained in [20], the traveling wave solution is expected to be unstable for a large number $N$. To prove the instability of the traveling wave solution, it is necessary to determine an unstable eigenvalue. However, the linearized eigenvalue problem is too difficult to analyze theoretically even in the case of $N=1$. From this point of view, we must reduce (4) and derive a simpler system. This is the motivation of our study.

Now, we state our main result in a formal way: the dynamics of $(N+1)$ camphor boats described in (4) can be reduced to a finite dimensional dynamical system under (A1)-(A3) if the length of the circuit is sufficiently large and each particle is sufficiently separated from other particles. We will describe the precise statement of our main theorem at the end of Section 5 (see Theorem 1).

Main result. If $L$ is sufficiently large and the initial value $U_{0}$ of (4) is close to $S\left(z, l_{0}, \mathbf{h}_{0}, \mathbf{r}_{0}\right)$ for some $l_{0}, \mathbf{h}_{0}, \mathbf{r}_{0}$, then the solution $U(z, t)$ of (4) can be approximated by $S(z, l, \mathbf{h}, \mathbf{r})$ for $t>0$, and $(l, \mathbf{h}, \mathbf{r})=(l(t), \mathbf{h}(t), \mathbf{r}(t))$ is governed by

$$
\left\{\begin{array}{l}
\frac{d l}{d t}=r_{0}+\text { h.o.t. } \\
\frac{d h_{i}}{d t}=r_{i+1}-r_{i}+\text { h.o.t. } \\
\frac{d r_{i}}{d t}=\text { h.o.t. }
\end{array}\right.
$$

where $r_{i}$ denotes the $i$-th component of $\mathbf{r}$ for $i=0, \ldots, N$ and $r_{N+1}$ corresponds to $r_{0}$.

Our main result is similar to that in [7], where the authors considered the interaction between two pulses with very small velocity near a bifurcation point in a reaction-diffusion system. In the aforementioned article, each pulse can be approximated by a stationary solution, which indicates that all eigenfunctions $\Phi, \Psi, \Phi^{*}, \Psi^{*}$ are expected to be symmetric, i.e., odd or even functions. Consequently, several calculations in the reduction process become simpler than in our case. $\Phi^{*}$ and $\Psi^{*}$ in our case will be introduced in the following section.

In the current study, we only have the lowest-order terms in reduced ordinary differential equations. We have a reduced system with higher-order terms derived in a formal way in [4]. We also derive a simpler system by an additional approximation and perform numerical simulations [5]. We will show that the system exhibits congestion of particles as shown in the experiment in [20]. However, we need more 
careful calculations in order to derive higher-order terms rigorously as obtained in [3] and [7], which will be undertaken in our future works.

Let $L(\mathbf{h}) \equiv \mathcal{L}^{\prime}(P(z, 0, \mathbf{h}), 0)$ be a linearized operator of $\mathcal{L}(U, 0)$ at $U=P(z, l, \mathbf{h})$. Using the resolvent operator of $L(\mathbf{h})$, we will define projection maps $Q(\mathbf{h})$ and $R(\mathbf{h}) \equiv I-Q(\mathbf{h})$ by the Dunford integral (see [15]). Here, we denote the identity map by $I: X \rightarrow X$. To derive a reduced system, we introduce a homeomorphism map $\Pi(\mathbf{h})$ such that $\Pi(\mathbf{h})^{-1} E(\mathbf{h})$ and $\Pi(\mathbf{h})^{-1} E^{\perp}(\mathbf{h})$ are independent of $\mathbf{h} \in H\left(h^{*}\right)$, where $E(\mathbf{h}) \equiv Q(\mathbf{h}) X$ and $E^{\perp}(\mathbf{h}) \equiv R(\mathbf{h}) X$. We note that $L(\mathbf{h})$ in this study includes delta functions, which causes the lack of regularity of functions and consequently makes it impossible to define the homeomorphism $\Pi(\mathbf{h})$ in a standard way as in [3]. We overcome it by applying a "discrete method" to the definition of $\Pi(\mathbf{h})$ in Section 3.

Further, weak regularity of functions causes other technical difficulties in theoretical approaches. In our analysis, we consider the linearized operator $L(\mathbf{h})$ in $X$ as a base space whereas the authors in [3] and [7] considered a usual Lebesgue space $L^{2}$. Hence, we must derive several properties of operators in $X$ as wellknown results in $L^{2}$ to estimate the resolvent operator of $L(\mathbf{h})$ in $X$, to clarify several properties of the fractional operator of $L(\mathbf{h})$ for some $\mathbf{h}$, etc. Moreover, it is complicated to pay careful attention to the regularity and check the dependency of parameters on those estimates. Our first aim in this study is to overcome the mathematical difficulties described above.

We transform (4) into a system (56), (57) of a pair of $(l, \mathbf{h}, \mathbf{r})$ and a function $W$, in which nonlinear terms include delta functions and may not be Lipschitz continuous. As the right-hand side of the first equation of (56) depends on only $\mathbf{h}, \mathbf{r}, W$, the system (56), (57) is equivalent to (60). Eventually, we will show that $W$ is sufficiently small. Thus, we can formally set $W=0$ in (60) and derive a reduced system for only $\mathbf{h}, \mathbf{r}$, de which is a similar result to that in [3] and [7]. It is our second aim in this study to derive (60) rigorously. Unfortunately, we cannot construct a center manifold in (60) in the same way as in [3] and [7] because the lack of regularity of functions makes it difficult to establish the uniqueness of the solution in (60). This study is a first step to overcome this problem in the near future.

In the present study we establish a new center manifold approach in $\left(H^{1}\right)^{*}$ framework in order to overcome mathematical difficulties described above in the reduction process. Such difficulties can be seen in interface equations arising in reaction-diffusion systems under a singular limit ([1], [12], [13],[19]). We expect that our new approach is applicable for these problems in one dimension. However, it is difficult to apply it for higher-dimensional problems directly. In spite that the almost all mathematical results in this manuscript are verified by using the Hölder continuity of functions, the Sobolev space $H^{1}$ cannot be imbedded into a usual Hölder space with any exponent in higher dimension (see Section 5.6 in [8]). We will challenge developing a center manifold theory in our future works to treat problems as in [6].

The rest of this paper is organized as follows. In Section 2 we introduce several notations which we use throughout this manuscript. Moreover, we describe the properties of $\mathcal{L}_{\infty}^{\prime}\left(0,0, p ; c_{0}, \mu_{0}, s_{0}\right)$ and $L(\mathbf{h})$, and prove in Lemmas 1,2 that $-\mathcal{L}_{\infty}^{\prime}\left(0,0, p ; c_{0}, \mu_{0}, s_{0}\right)$ and $-L(\mathbf{h})$ are sectorial operators (see [11] for the definition of sectorial operators). In Lemma 3 , the fractional space induced by $L(\mathbf{h})$ can be embedded into a usual Lebesgue space and a Hölder space. In Section 3 we 
study the regularity of the projection mappings $Q(\mathbf{h})$ and $R(\mathbf{h})$ with respect to $\mathbf{h}$, and verify that they are Hölder continuous (Lemmas 4-10). Using this fact, we define a homeomorphism $\Pi(\mathbf{h})$. Finally, we prove the Hölder continuity of $\Pi(\mathbf{h})$ and its inverse $\Pi(\mathbf{h})^{-1}$ (Lemma 12). In Section 4 we define an evolution operator $T\left(t, t_{0} ; \mathbf{h}\right)$ generated by $\tilde{L}(\mathbf{h}) \equiv \Pi(\mathbf{h})^{-1} L(\mathbf{h}) \Pi(\mathbf{h})$ by a standard theory in [11], and have the decaying property of $T\left(t, t_{0} ; \mathbf{h}\right)$ (see (43)). In Section 5 we show that the system (4) can be decomposed into a finite dimensional manifold coordinated by $(l, \mathbf{h}, \mathbf{r})$ and its normal direction, denoted by $W$ (Lemma 18). Consequently, we will derive a system (60) and estimate $W$. Finally we prove our main result, Theorem 1.

\section{Preliminaries}

We define several notations. We set $H\left(h^{*}\right) \equiv\left\{\mathbf{h}={ }^{t}\left(h_{0}, \ldots, h_{N-1}\right) \in \mathbb{R}^{N} \mid h^{*}<\right.$ $\left.h_{j}(j=0, \ldots, N)\right\}$. If $h_{1}^{*}$ and $h_{2}^{*}$ satisfy $h_{1}^{*}<h_{2}^{*}$ and $(6), H\left(h_{2}^{*}\right) \subset H\left(h_{1}^{*}\right)$ holds true. We set $\mathbf{h}^{*} \equiv{ }^{t}(L /(N+1), \ldots, L /(N+1)) \in \mathbb{R}^{N}$ and observe that $\mathbf{h}^{*}$ is included in $H\left(h^{*}\right)$ for any $h^{*}$ with (6). Consequently, we simply estimate

$$
\left|\mathbf{h}_{1}-\mathbf{h}_{2}\right| \leq\left|\mathbf{h}_{1}-\mathbf{h}^{*}\right|+\left|\mathbf{h}_{2}-\mathbf{h}^{*}\right| \leq 2 C_{*} \sqrt{N}
$$

uniformly in $\mathbf{h}_{1}, \mathbf{h}_{2} \in H\left(h^{*}\right)$ based on (6). Set $R\left(r^{*}\right) \equiv\left\{\mathbf{r}={ }^{t}\left(r_{0}, \ldots, r_{N}\right) \in\right.$ $\left.\mathbb{R}^{N+1} \mid r_{j}<r^{*}(j=0, \ldots, N)\right\}$ for a constant $r^{*}>0$. We represent the $m \times m$ identity matrix by $I_{m}$ for $m \in \mathbb{N}$. We simply denote the identity maps in several Banach spaces by $I$ without distinguishing them. We denote the derivative of $W \in X$ with respect to $z$ by $\partial W / \partial z={ }^{t}\left(0,0, \ldots, 0,0, w^{\prime}(z)\right)$, where $w(z)$ is the $(2 N+3)$-th component of $W$ and $w^{\prime}(z)$ denotes the derivative of $w$ if it can be defined.

Generally, we denote a norm of a Banach space $B$ by $\|\cdot\|_{B}$ and a usual Euclidian norm by $|\cdot|$. We denote $\langle\cdot, \cdot\rangle_{\infty}$ by a pairing between $\mathbb{R}^{2} \times H^{1}(\mathbb{R})$ and $\mathbb{R}^{2} \times\left(H^{1}(\mathbb{R})\right)^{*}$. Thus, the norm $\|\cdot\|_{\mathbb{R}^{2} \times\left(H^{1}(\mathbb{R})\right)^{*}}$ can be given by

$$
\left\|\varphi^{*}\right\|_{\mathbb{R}^{2} \times\left(H^{1}(\mathbb{R})\right)^{*}} \equiv \sup \left\{\left|\left\langle\varphi^{*}, \varphi\right\rangle_{\infty}\right| \mid \varphi \in \mathbb{R}^{2} \times H^{1}(\mathbb{R}),\|\varphi\|_{\mathbb{R}^{2} \times H^{1}(\mathbb{R})}=1\right\}
$$

for $\varphi^{*} \in \mathbb{R}^{2} \times\left(H^{1}(\mathbb{R})\right)^{*}$. We denote a pairing between $X$ and $X^{1}$ by $\langle\cdot, \cdot\rangle$ for simplicity. Then we can provide a norm in $X$ as above. Note that $X=\left(X^{1}\right)^{*}$. We may use a notation $\langle\cdot, \cdot\rangle_{H^{1}(\mathbb{R})}$, which is a pairing between $\left(H^{1}(\mathbb{R})\right)^{*}$ and $H^{1}(\mathbb{R})$. Similarly, $\langle\cdot, \cdot\rangle_{H^{1}(\mathbb{T})}$ denotes a pairing between $\left(H^{1}(\mathbb{T})\right)^{*}$ and $H^{1}(\mathbb{T})$. Finally, we represent a usual operator norm simply by $\|\cdot\|$.

Let $L^{q}(\mathbb{R})$ and $L^{q}(\mathbb{T})$ be the usual Lebesgue spaces on $\mathbb{R}$ and $\mathbb{T}$ for $1 \leq q \leq \infty$, respectively. It is well known that $L^{2}(\mathbb{R})$ can be embedded into $\left(H^{1}(\mathbb{R})\right)^{*}$ by the inclusion map $\iota$. More precisely, $\iota u \in\left(H^{1}(\mathbb{R})\right)^{*}$ for $u \in L^{2}(\mathbb{R})$ can be defined by

$$
\langle\iota u, \phi\rangle_{H^{1}(\mathbb{R})}=\int_{-\infty}^{\infty} u(z) \phi(z) d z
$$

for $\phi \in H^{1}(\mathbb{R})$. As $L^{2}(\mathbb{R}) \subset\left(H^{1}(\mathbb{R})\right)^{*}$ in this sense, we do not distinguish between $\iota u$ and $u$ throughout this article for simplicity. Similarly, $L^{2}(\mathbb{T}) \subset\left(H^{1}(\mathbb{T})\right)^{*}$ holds true. Finally, we denote a Banach space consisting of Hölder continuous functions on $\mathbb{T}$ with a Hölder exponent $\zeta$ by $C^{\zeta}(\mathbb{T})$. 
Next we study spectra and estimate resolvent operators of $\mathcal{L}_{\infty}^{\prime}\left(0,0, p ; c_{0}, \mu_{0}, s_{0}\right)$ and $L(\mathbf{h})$. Hereafter, we denote general constants by $C$ independent of $\lambda, L, h^{*}$ and $\mathbf{h}$. We first study $\mathcal{L}_{\infty}^{\prime}\left(0,0, p ; c_{0}, \mu_{0}, s_{0}\right)$. We refer to [15] for the definition of Fredholm operators.

Lemma 1 There are $\theta>\pi / 2$ in the definition of $\Sigma$ in the assumption (A3), $M>0$ and $C>0$ such that $\mathcal{L}_{\infty}^{\prime}\left(0,0, p ; c_{0}, \mu_{0}, s_{0}\right)$ satisfies the following conditions;

(i) For $\lambda \in \Sigma, \lambda I-\mathcal{L}_{\infty}^{\prime}\left(0,0, p ; c_{0}, \mu_{0}, s_{0}\right)$ is a Fredholm operator.

(ii) Any $\lambda \in \Sigma$ with $|\lambda| \geq M$ belongs to the resolvent set of $\mathcal{L}_{\infty}^{\prime}\left(0,0, p ; c_{0}, \mu_{0}, s_{0}\right)$.

(iii) The resolvent estimate

$$
\left\|\left(\lambda I-\mathcal{L}_{\infty}^{\prime}\left(0,0, p ; c_{0}, \mu_{0}, s_{0}\right)\right)^{-1}\right\| \leq \frac{C}{|\lambda|}
$$

holds true uniformly in $\lambda \in \Sigma$ with $|\lambda| \geq M$.

Proof Define $A$ and $B$ by

$$
\begin{aligned}
& A U \equiv\left(\begin{array}{c}
0 \\
0 \\
\frac{\partial^{2} u}{\partial z^{2}}-c_{0} \frac{\partial u}{\partial z}-\alpha u
\end{array}\right) \\
& B U \equiv\left(\begin{array}{c}
Y \\
-\mu_{0} Y+\gamma^{\prime}(p(\rho))\left(p^{\prime}(\rho) Z+u(\rho)\right)-\gamma^{\prime}(p(-\rho))\left(p^{\prime}(-\rho) Z+u(-\rho)\right) \\
-Z\left[s_{0} \delta_{-\rho}-\left(s_{0}-1\right) \delta_{0}-\delta_{\rho}\right]
\end{array}\right)
\end{aligned}
$$

for $U={ }^{t}(Z, Y, u)$ with the domains $D(A)=\mathbb{R}^{2} \times H^{1}(\mathbb{R})$ and $D(B)=\mathbb{R}^{2} \times$ $\left(\left(H^{1}(\mathbb{R})\right)^{*} \cap C_{b}(\mathbb{R})\right)$, where $C_{b}(\mathbb{R})$ denotes a set of bounded and continuous functions on $\mathbb{R}$. The Sobolev's imbedding theorem implies $D(A) \subset D(B)$. We decompose the operator $\mathcal{L}_{\infty}^{\prime}\left(0,0, p ; c_{0}, \mu_{0}, s_{0}\right)$ into $\mathcal{L}_{\infty}^{\prime}\left(0,0, p ; c_{0}, \mu_{0}, s_{0}\right)=A+B$.

It is easy to see that $\lambda I-A$ is Fredholm for $\lambda \in \Sigma$ because $D(A)$ is dense in $\mathbb{R}^{2} \times\left(H^{1}(\mathbb{R})\right)^{*}$ and $\lambda I-A$ is a closed operator. In addition, for any $f \in\left(H^{1}(\mathbb{R})\right)^{*}$, there is a unique solution $u \in H^{1}(\mathbb{R})$ of $\lambda u-\left(u^{\prime \prime}-c_{0} u^{\prime}-\alpha u\right)=f$ and there is $C>0$ independent of $\lambda, u, f$ such that

$$
\|u\|_{\left(H^{1}(\mathbb{R})\right)^{*}} \leq \frac{C}{|\lambda+\alpha|}\|f\|_{\left(H^{1}(\mathbb{R})\right)^{*}}, \quad\|u\|_{H^{1}(\mathbb{R})} \leq C\|f\|_{\left(H^{1}(\mathbb{R})\right)^{*}} .
$$

We next prove that $B$ is $(\lambda I-A)$-compact (see Section 1 in Chapter IV in [15] for the definition of $T$-compact). Suppose that for $\Phi_{n}={ }^{t}\left(Z_{n}, Y_{n}, u_{n}\right) \in D(A)$, there is a constant $C>0$ independent of $n$ such that

$$
\left\|\Phi_{n}\right\|_{\mathbb{R}^{2} \times\left(H^{1}(\mathbb{R})\right)^{*}}+\left\|(\lambda I-A) \Phi_{n}\right\|_{\mathbb{R}^{2} \times\left(H^{1}(\mathbb{R})\right)^{*}} \leq C .
$$

Set $f_{n}=\lambda u_{n}-\left(u_{n}^{\prime \prime}-c_{0} u_{n}^{\prime}-\alpha u_{n}\right) \in\left(H^{1}(\mathbb{R})\right)^{*}$. From the inequality above and (8), we see that $\left|Z_{n}\right|,\left|Y_{n}\right|$, and $\left\|u_{n}\right\|_{H^{1}(\mathbb{R})}$ are uniformly bounded in $n$. Then there are a subsequence $\left(Z_{n_{k}}, Y_{n_{k}}, u_{n_{k}}\right)$ and $(Z, Y, u) \in \mathbb{R}^{2} \times H^{1}(\mathbb{R})$ such that $\left(Z_{n_{k}}, Y_{n_{k}}, u_{n_{k}}\right) \rightarrow(Z, Y, u)$ weakly in $\mathbb{R}^{2} \times H^{1}(\mathbb{R})$ as $k \rightarrow \infty$. Furthermore we can assume that $u \in C_{b}(\mathbb{R})$ and $u_{n_{k}}(z) \rightarrow u(z)$ uniformly in $[-R, R]$ as $k \rightarrow \infty$ for sufficiently large $R>0$ without loss of generality by the Sobolev's imbedding 
theorem. Therefore $B \Phi_{n_{k}}$ converges to $B \Phi$ as $k \rightarrow \infty$. Hence we complete the proof of Lemma 1 (i) by Theorem 5.26 in Chapter IV in [15].

Next we prove (ii), (iii). Suppose that for $W \in \mathbb{R}^{2} \times\left(H^{1}(\mathbb{R})\right)^{*}$, there is a solution $U$ of $\left(\lambda I-\mathcal{L}_{\infty}^{\prime}\left(0,0, p ; c_{0}, \mu_{0}, s_{0}\right)\right) U=W$. Put $U={ }^{t}(Z, Y, u)$. From (8), we have

$$
\begin{aligned}
& |Z| \leq \frac{1}{|\lambda|}\left(|Y|+\|W\|_{\mathbb{R}^{2} \times\left(H^{1}(\mathbb{R})\right)^{*}}\right), \\
& |Y| \leq \frac{C}{\left|\lambda+\mu_{0}\right|}\left(|Z|+\|u\|_{H^{1}(\mathbb{R})}+\|W\|_{\mathbb{R}^{2} \times\left(H^{1}(\mathbb{R})\right)^{*}}\right), \\
& \|u\|_{H^{1}(\mathbb{R})} \leq C\left(\|W\|_{\mathbb{R}^{2} \times\left(H^{1}(\mathbb{R})\right)^{*}}+|Z|\right), \\
& \|u\|_{\left(H^{1}(\mathbb{R})\right)^{*}} \leq \frac{C}{|\lambda+\alpha|}\left(\|W\|_{\mathbb{R}^{2} \times\left(H^{1}(\mathbb{R})\right)^{*}}+|Z|\right) .
\end{aligned}
$$

From these inequalities, there is a constant $C$ independent of $\lambda, W, M$ such that

$$
\|U\|_{\mathbb{R}^{2} \times L^{\infty}(\mathbb{R})} \leq C\|W\|_{\mathbb{R}^{2} \times\left(H^{1}(\mathbb{R})\right)^{*}}, \quad\|U\|_{\mathbb{R}^{2} \times\left(H^{1}(\mathbb{R})\right)^{*}} \leq \frac{C}{|\lambda|}\|W\|_{\mathbb{R}^{2} \times\left(H^{1}(\mathbb{R})\right)^{*}}
$$

uniformly in $\lambda \in \Sigma$ with $|\lambda| \geq M$ for sufficiently large $M$. The second inequality above implies that any $\lambda \in \Sigma$ with $|\lambda| \geq M$ is included in the resolvent set of $\lambda I-\mathcal{L}_{\infty}^{\prime}\left(0,0, p ; c_{0}, \mu_{0}, s_{0}\right)$.

Remark 1 For $f \in\left(H^{1}(\mathbb{T})\right)^{*}$ and $\lambda \in \Sigma$, let $u \in H^{1}(\mathbb{T})$ be a solution of $\lambda u-\left(u^{\prime \prime}-\right.$ $\left.c_{0} u^{\prime}-\alpha u\right)=f$. It is well-known that the following estimates hold true;

$$
\|u\|_{\left(H^{1}(\mathbb{T})\right)^{*}} \leq \frac{C}{|\lambda+\alpha|}\|f\|_{\left(H^{1}(\mathbb{T})\right)^{*}}, \quad\|u\|_{H^{1}(\mathbb{T})} \leq C\|f\|_{\left(H^{1}(\mathbb{T})\right)^{*}}
$$

for a constant $C$ independent of $L, \lambda, u, f$.

In the following, $\theta$ denotes a constant given in Lemma 1 throughout this article. Lemma 1 implies that $\lambda \in \Sigma$ either belongs to the resolvent set or is a spectrum with a finite multiplicity of $\mathcal{L}_{\infty}^{\prime}\left(0,0, p ; c_{0}, \mu_{0}, s_{0}\right)$, which is called an "eigenvalue". Lemma 1 implies that all spectra in $\Sigma$ of $\mathcal{L}_{\infty}^{\prime}\left(0,0, p ; c_{0}, \mu_{0}, s_{0}\right)$ should satisfy $|\lambda| \leq$ $M$. Assumption (A3) implies that $\lambda \in \Sigma$ with $|\lambda| \leq M$ belongs to the resolvent set of $\mathcal{L}_{\infty}^{\prime}\left(0,0, p ; c_{0}, \mu_{0}, s_{0}\right)$ except for $\lambda=0$. In addition, $\lambda=0$ is an eigenvalue and its multiplicity is 2 due to (A2). These facts and a decomposition theorem described in [15] give the following resolvent estimates;

$$
\left\|\left(\lambda I-\mathcal{L}_{\infty}^{\prime}\left(0,0, p ; c_{0}, \mu_{0}, s_{0}\right)\right)^{-1}\right\| \leq \begin{cases}C, & \lambda \in \Sigma, m \leq|\lambda| \leq M, \\ \frac{C}{|\lambda|^{2}}, & \lambda \in \Sigma,|\lambda| \leq m\end{cases}
$$

for small $m>0$, where $C$ may depend on $m$.

In the reduction process, we will project the solution $U(z, t)$ of $(4)$ onto a finite dimensional manifold coordinated by $\mathbf{h} \in H\left(h^{*}\right)$. According to [3], the tangent space of the manifold, denoted by $E(\mathbf{h})$, will be represented by the generalized eigenfunctions of $\mathcal{L}_{\infty}^{\prime}\left(0,0, p ; c_{0}, \mu_{0}, s_{0}\right)$ and $\left(\mathcal{L}_{\infty}^{\prime}\left(0,0, p ; c_{0}, \mu_{0}, s_{0}\right)\right)^{*}$, which is associated with the adjoint operator of $\mathcal{L}_{\infty}^{\prime}\left(0,0, p ; c_{0}, \mu_{0}, s_{0}\right)$ and can be explicitly 
given by

$$
\begin{aligned}
& \left(\mathcal{L}_{\infty}^{\prime}\left(0,0, p ; c_{0}, \mu_{0}, s_{0}\right)\right)^{*} \Phi^{*} \\
& \equiv\left(\begin{array}{c}
\gamma_{0} Y_{\Phi}^{*}-\left[s_{0} \phi^{*}(-\rho)-\left(s_{0}-1\right) \phi^{*}(0)-\phi^{*}(\rho)\right] \\
Z_{\Phi}^{*}-\mu_{0} Y_{\Phi}^{*} \\
\frac{\partial^{2} \phi^{*}}{\partial z^{2}}+c_{0} \frac{\partial \phi^{*}}{\partial z}-\alpha \phi^{*}+\left(\gamma^{\prime}(p(\rho)) \delta_{\rho}-\gamma^{\prime}(p(\rho)) \delta_{-\rho}\right) Y_{\Phi}^{*}
\end{array}\right)
\end{aligned}
$$

for $\Phi^{*}=\left(Z_{\Phi}^{*}, Y_{\Phi}^{*}, \phi^{*}\right) \in \mathbb{R}^{2} \times H^{1}(\mathbb{R})$, where $\gamma_{0}$ is a constant defined by $\gamma_{0} \equiv$ $\gamma^{\prime}(p(\rho)) p^{\prime}(\rho)-\gamma^{\prime}(p(-\rho)) p^{\prime}(-\rho)$. Using this expression, we have

$$
\left\langle\mathcal{L}_{\infty}^{\prime}\left(0,0, p ; c_{0}, \mu_{0}, s_{0}\right) \Phi, \Phi^{*}\right\rangle_{\infty}=\left\langle\left(\mathcal{L}_{\infty}^{\prime}\left(0,0, p ; c_{0}, \mu_{0}, s_{0}\right)\right)^{*} \Phi^{*}, \Phi\right\rangle_{\infty}
$$

for $\Phi, \Phi^{*} \in \mathbb{R}^{2} \times H^{1}(\mathbb{R})$. Since $\mathbb{R}^{2} \times H^{1}(\mathbb{R})$ is reflective, $\left(\mathcal{L}_{\infty}^{\prime}\left(0,0, p ; c_{0}, \mu_{0}, s_{0}\right)\right)^{*}$ is thought of as an operator in $\mathbb{R}^{2} \times\left(H^{1}(\mathbb{R})\right)^{*}$ with a domain $\mathbb{R}^{2} \times H^{1}(\mathbb{R})$.

Let $\Phi^{*}, \Psi^{*}$ be the generalized eigenfunctions of $\left(\mathcal{L}_{\infty}^{\prime}\left(0,0, p ; c_{0}, \mu_{0}, s_{0}\right)\right)^{*}$ which satisfy $\left(\mathcal{L}_{\infty}^{\prime}\left(0,0, p ; c_{0}, \mu_{0}, s_{0}\right)\right)^{*} \Phi^{*}=0$ and $\left(\mathcal{L}_{\infty}^{\prime}\left(0,0, p ; c_{0}, \mu_{0}, s_{0}\right)\right)^{*} \Psi^{*}=-\Phi^{*}$, respectively. Under the assumptions (A1)-(A3), $\Phi^{*}, \Psi^{*}$ do exist thanks to the RiezSchauder's theorem (see [15]). Then, $\Phi^{*}$ and $\Psi^{*}$ are uniquely determined by orthogonal conditions such as $\left\langle\Psi, \Phi^{*}\right\rangle_{\infty}=1$ and $\left\langle\Psi, \Psi^{*}\right\rangle_{\infty}=0$. Then we note that $\left\langle\Phi, \Psi^{*}\right\rangle_{\infty}=1$ and $\left\langle\Phi, \Phi^{*}\right\rangle_{\infty}=0$ automatically hold. Put $\Phi^{*}={ }^{t}\left(Z_{\Phi}^{*}, Y_{\Phi}^{*}, \phi^{*}\right)$ and $\Psi^{*}={ }^{t}\left(Z_{\Psi}^{*}, Y_{\Psi}^{*}, \psi^{*}\right)$. Note that $\phi, \psi, \phi^{*}, \psi^{*}$ decay exponentially as $|z| \rightarrow \infty$. More precisely, there is $\beta_{1}>0$ such that $|\varphi(z)| \leq C e^{-\beta_{1}|z|}$ and $\left|\varphi^{\prime}(z)\right| \leq C e^{-\beta_{1}|z|}$ in $z \in \mathbb{R}$ for $\varphi=p, \phi, \psi, \phi^{*}, \psi^{*}$.

Next we study $L(\mathbf{h})$. It is easy to see that $\lambda I-L(\mathbf{h})$ is Fredholm in $X$ for $\lambda \in \Sigma$ by the same proof as that of Lemma 1 . Next we study the spectra and the resolvent of $L(\mathbf{h})$ in the following lemma. We prove it for the readers though it is the same as in [3] and [7]. Here we set $\varepsilon \equiv e^{-\beta_{1} h^{*}}$. If $h^{*}$ is large, $\varepsilon$ is small.

Lemma 2 There exist positive constants $C_{1}, C_{2}, C_{3}, M$ independent of $h^{*}$ such that if $h^{*}$ is sufficiently large, then $L(\mathbf{h})$ satisfies the following facts;

(i) The resolvent estimate

$$
\left\|(\lambda I-L(\mathbf{h}))^{-1} U\right\|_{X^{1}} \leq C_{2}\left(1+\frac{1}{|\lambda|}\right)^{2}\|U\|_{X}
$$

holds true uniformly in $\mathbf{h} \in H\left(h^{*}\right), \lambda \in \Sigma$ with $C_{1} \varepsilon^{1 / 8} \leq|\lambda|$ and $U \in X$.

(ii) $L(\mathbf{h})$ satisfies

$$
\left\|(\lambda I-L(\mathbf{h}))^{-1}\right\| \leq \frac{C_{3}}{|\lambda|}, \quad\left\|(\lambda I-L(\mathbf{h}))^{-1} U\right\|_{X^{1}} \leq C_{3}\|U\|_{X}
$$

uniformly in $\mathbf{h} \in H\left(h^{*}\right), \lambda \in \Sigma$ with $|\lambda| \geq M$ and $U \in X$.

The inequality (12) can be proved by the same way as in Lemma 1 . So we only consider the case of $|\lambda| \leq M$ for $\lambda \in \Sigma$ and prove (11). Actually, we can prove that the total number of eigenvalues of $L(\mathbf{h})$ close to 0 is exactly equal to $2(N+1)$. Remember that the number of eigenvalues, what is called "algebraic multiplicity", corresponds to the dimension of the associated eigenspace $E(\mathbf{h})$ (see [15]). We will define $E(\mathbf{h})$ in the next section precisely and show the facts above (see Remark 2). 
Here we introduce

$$
\begin{aligned}
& {\left[\hat{\Phi}_{k}(\mathbf{h})\right](z) \equiv-e_{2 k+1}+\bar{\phi}\left(z-\bar{z}_{k}\right) e_{2 N+3}} \\
& {\left[\hat{\Psi}_{k}(\mathbf{h})\right](z) \equiv e_{2 k+2}+\bar{\psi}\left(z-\bar{z}_{k}\right) e_{2 N+3}} \\
& {\left[\hat{\Phi}_{k}^{*}(\mathbf{h})\right](z) \equiv Z_{\Phi}^{*} e_{2 k+1}+Y_{\Phi}^{*} e_{2 k+2}+\overline{\phi^{*}}\left(z-\bar{z}_{k}\right) e_{2 N+3}} \\
& {\left[\hat{\Psi}_{k}^{*}(\mathbf{h})\right](z) \equiv Z_{\Psi}^{*} e_{2 k+1}+Y_{\Psi}^{*} e_{2 k+2}+\overline{\psi^{*}}\left(z-\bar{z}_{k}\right) e_{2 N+3}}
\end{aligned}
$$

for $k=0, \ldots, N$, which play an important role in the proof of Lemma 2. Actually, those functions approximate eigenfunctions of $L(\mathbf{h})$ and $L(\mathbf{h})^{*}$, which we will claim in Lemmas 4 and 8 more precisely. Here $L(\mathbf{h})^{*}$ is an operator from $X^{1} \rightarrow X$ defined by an equality $\left\langle L(\mathbf{h}) \Phi, \Phi^{*}\right\rangle=\left\langle L(\mathbf{h})^{*} \Phi^{*}, \Phi\right\rangle$ for $\Phi, \Phi^{*} \in X^{1}$, which is associated with the adjoint operator of $L(\mathbf{h})$.

Proof We first suppose that there exists a solution $U$ of $(\lambda I-L(\mathbf{h})) U=\hat{\Phi}_{k}(\mathbf{h})$ for $k=0, \ldots, N, \lambda \in \Sigma$ with $|\lambda| \leq M$ for sufficiently large $M$. We set $U(z)=$ ${ }^{t}\left(z_{0}, y_{0}, \ldots, z_{N}, y_{N}, u(z)\right)$ and

$$
\begin{aligned}
& U_{i}(z) \equiv z_{i} e_{2 i+1}+y_{i} e_{2 i+2}+\bar{\zeta}(\nu z) u\left(z+\bar{z}_{i}\right) e_{2 N+3} \quad(i=0, \ldots, N), \\
& \tilde{U}(z) \equiv\left(1-\sum_{i=0}^{N} \bar{\zeta}\left(\nu\left(z-\bar{z}_{i}\right)\right)\right) u(z) e_{2 N+3}
\end{aligned}
$$

where $\zeta$ is a cut-off function satisfying $\zeta(z)=1$ if $|z| \leq 1$ and $\zeta(z)=0$ if $|z| \geq 2$, and $\nu \equiv 4 / h^{*}$. Then we have $U(z)=\sum_{i=0}^{N} U_{i}\left(z-\bar{z}_{i}\right)+\tilde{U}(z)$.

We first estimate $\tilde{U}$. Put $\tilde{u}(z) \equiv\left(1-\sum_{i=0}^{N} \bar{\zeta}\left(\nu\left(z-\bar{z}_{i}\right)\right)\right) u(z)$. Then $\tilde{u}$ satisfies $\lambda \tilde{u}-\left(\tilde{u}^{\prime \prime}-c_{0} \tilde{u}^{\prime}-\alpha \tilde{u}\right)=F(z)$, where $F=F(z)$ is defined by

$$
\begin{aligned}
F & \equiv \sum_{i=0}^{N}\left[\nu^{2} \bar{\zeta}^{\prime \prime}\left(\nu\left(z-\bar{z}_{i}\right)\right) u(z)+2 \nu \bar{\zeta}^{\prime}\left(\nu\left(z-\bar{z}_{i}\right)\right) u^{\prime}(z)-c_{0} \nu \bar{\zeta}^{\prime}\left(\nu\left(z-\bar{z}_{i}\right)\right) u(z)\right] \\
& +\left(1-\sum_{i=0}^{N} \bar{\zeta}\left(\nu\left(z-\bar{z}_{i}\right)\right)\right) \bar{\phi}\left(z-\bar{z}_{k}\right)
\end{aligned}
$$

By (9), we have $\|\tilde{u}\|_{H^{1}(\mathbb{T})} \leq C\|F\|_{\left(H^{1}(\mathbb{T})\right)^{*}} \leq C\left(\varepsilon^{1 / 4}+\nu\|u\|_{H^{1}(\mathbb{T} \backslash B)}\right)$. Here we set $B \equiv \cup_{i=0}^{N} B_{1 / \nu}\left(\bar{z}_{i}\right)$, where $B_{r}(z)$ denotes a ball with the radius $r$ and the center $z$.

Put $u_{i}(z) \equiv \bar{\zeta}(\nu z) u\left(z+\bar{z}_{i}\right)$ and $\tilde{U}_{i} \equiv{ }^{t}\left(z_{i}, y_{i}, u_{i}\right)$ for $i=0, \ldots, N$. For $i=k$, we have $\lambda z_{k}-y_{k}=-1$,

$\lambda y_{k}-\left(-\mu_{0} y_{k}+\gamma^{\prime}(p(\rho))\left(p^{\prime}(\rho) z_{k}+u_{k}(\rho)\right)-\gamma^{\prime}(p(-\rho))\left(p^{\prime}(-\rho) z_{k}+u_{k}(-\rho)\right)\right)=F_{1}$, and

$$
\lambda u_{k}-\left(u_{k}^{\prime \prime}-c_{0} u_{k}^{\prime}-\alpha u_{k}-z_{k}\left[s_{0} \delta_{-\rho}-\left(s_{0}-1\right) \delta_{0}-\delta_{\rho}\right]\right)=\bar{\zeta}(\nu z) \bar{\phi}(z)+F_{2}(z),
$$

where $F_{1}, F_{2}(z)$ are defined by

$$
\begin{aligned}
F_{1} & \equiv \gamma^{\prime}\left(\sum_{i=0}^{N} \bar{p}\left(\bar{z}_{k}-\bar{z}_{i}+\rho\right)\right)\left(\sum_{i=0}^{N} \bar{p}^{\prime}\left(\bar{z}_{k}-\bar{z}_{i}+\rho\right) z_{i}+u_{k}(\rho)\right) \\
& -\gamma^{\prime}\left(\sum_{i=0}^{N} \bar{p}\left(\bar{z}_{k}-\bar{z}_{i}-\rho\right)\right)\left(\sum_{i=0}^{N} \bar{p}^{\prime}\left(\bar{z}_{k}-\bar{z}_{i}-\rho\right) z_{i}+u_{k}(-\rho)\right) \\
& -\gamma^{\prime}(p(\rho))\left(p^{\prime}(\rho) z_{k}+u_{k}(\rho)\right)+\gamma^{\prime}(p(-\rho))\left(p^{\prime}(-\rho) z_{k}+u_{k}(-\rho)\right),
\end{aligned}
$$




$$
F_{2}(z) \equiv-\nu^{2} \bar{\zeta}^{\prime \prime}(\nu z) u\left(z+\bar{z}_{k}\right)-2 \nu \bar{\zeta}^{\prime}(\nu z) u^{\prime}\left(z+\bar{z}_{k}\right)+c_{0} \nu \bar{\zeta}^{\prime}(\nu z) u\left(z+\bar{z}_{k}\right)
$$

Here we naturally extend the domains of $u_{k}(z)$ and $F_{2}(z)$ to a whole line $\mathbb{R}$ by setting $u_{k}(z) \equiv 0$ and $F_{2}(z) \equiv 0$ in $\left(-\infty,-h^{*} / 2\right) \cup\left(h^{*} / 2, \infty\right)$. Then the equations above are represented by $\left(\lambda I-\mathcal{L}_{\infty}^{\prime}\left(0,0, p ; c_{0}, \mu_{0}, s_{0}\right)\right) \tilde{U}_{k}=Y_{1}+Y_{2}$, where $Y_{1} \equiv$ ${ }^{t}(-1,0, \zeta(\nu z) \phi(z))$ and $Y_{2} \equiv{ }^{t}\left(0, F_{1}, F_{2}(z)\right)$. Here we have

$$
\begin{array}{ll}
\left|\left\langle Y_{1}, \Phi^{*}\right\rangle_{\infty}\right| \leq C \varepsilon^{1 / 2}, & \left|\left\langle Y_{1}, \Psi^{*}\right\rangle_{\infty}-1\right| \leq C \varepsilon^{1 / 2}, \\
\left|\left\langle Y_{2}, \Phi^{*}\right\rangle_{\infty}\right| \leq C N_{1}, & \left|\left\langle Y_{2}, \Psi^{*}\right\rangle_{\infty}\right| \leq C N_{1},
\end{array}
$$

where $N_{1}=\varepsilon^{1 / 4} \nu\|u\|_{H^{1}(\mathbb{T} \backslash B)}+\varepsilon\|U\|_{X^{1}}$. We can expand $\tilde{U}_{k}$ as $\tilde{U}_{k}=a \Phi+b \Psi+\tilde{U}_{k}^{\perp}$, where $a, b$ are given by $a \equiv\left\langle\tilde{U}_{k}, \Psi^{*}\right\rangle_{\infty}$ and $b \equiv\left\langle\tilde{U}_{k}, \Phi^{*}\right\rangle_{\infty}$, and $\tilde{U}_{k}^{\perp}$ satisfies $\left\langle\tilde{U}_{k}^{\perp}, \Phi^{*}\right\rangle_{\infty}=\left\langle\tilde{U}_{k}^{\perp}, \Psi^{*}\right\rangle_{\infty}=0$. Substituting it into the above equation, we have

$$
\left|\lambda a+b-\left\langle Y_{1}, \Psi^{*}\right\rangle_{\infty}\right| \leq C N_{1}, \quad\left|\lambda b-\left\langle Y_{1}, \Phi^{*}\right\rangle_{\infty}\right| \leq C N_{1}
$$

and

$$
\begin{aligned}
& \left(\lambda I-\mathcal{L}_{\infty}^{\prime}\left(0,0, p ; c_{0}, \mu_{0}, s_{0}\right)\right) \tilde{U}_{k}^{\perp} \\
& =Y_{1}+Y_{2}-\left\langle Y_{1}+Y_{2}, \Psi^{*}\right\rangle_{\infty} \Phi-\left\langle Y_{1}+Y_{2}, \Phi^{*}\right\rangle_{\infty} \Psi .
\end{aligned}
$$

From (A1)-(A3) and a similar result of Theorem 6.17 in Chapter 3 in [15], we have

$$
\left\|\tilde{U}_{k}^{\perp}\right\|_{\mathbb{R}^{2} \times H^{1}(\mathbb{R})} \leq C\left(\varepsilon^{1 / 4}+\nu\|u\|_{H^{1}(\mathbb{T} \backslash B)}+\varepsilon\|U\|_{X^{1}}\right) .
$$

Then we have $\left\|\tilde{U}_{k}-\Phi / \lambda\right\|_{\mathbb{R}^{2} \times H^{1}(\mathbb{R})} \leq C N_{2}$ by putting

$$
N_{2} \equiv\left(1+\frac{\varepsilon^{1 / 4}}{|\lambda|}\left(1+\frac{1}{|\lambda|}\right)\right)\left(\varepsilon^{1 / 4}+\nu\|u\|_{H^{1}(\mathbb{T} \backslash B)}\right)+C\left(1+\frac{1}{|\lambda|}\right)^{2} \varepsilon\|U\|_{X^{1}}
$$

By the similar argument, we have $\left\|\tilde{U}_{i}\right\|_{\mathbb{R}^{2} \times H^{1}(\mathbb{R})} \leq C N_{2}$ for any $i \neq k$. Hence we have $\left\|U-\hat{\Phi}_{k}(\mathbf{h}) / \lambda\right\|_{X^{1}} \leq C N_{2}$. Here we have

$$
\left\|U-\frac{1}{\lambda} \hat{\Phi}_{k}(\mathbf{h})\right\|_{X^{1}} \geq\|u\|_{H^{1}(\mathbb{T} \backslash B)}-\frac{C}{|\lambda|} \varepsilon^{1 / 4} .
$$

Since $\nu$ is sufficiently small and fixed independently of $\varepsilon$, we have $\|u\|_{H^{1}(\mathbb{T} \backslash B)} \leq$ $C N_{3}$ and $\left\|U-\hat{\Phi}_{k}(\mathbf{h}) / \lambda\right\|_{X^{1}} \leq C N_{3}$ for any $\lambda$ with $C \varepsilon^{1 / 8} \leq|\lambda|$, where $N_{3} \equiv$ $\varepsilon^{1 / 8}+(1+1 /|\lambda|)^{2} \varepsilon\|U\|_{X^{1}}$. Therefore we have

$$
\left\|U-\frac{1}{\lambda} \hat{\Phi}_{k}(\mathbf{h})\right\|_{X^{1}} \leq C \varepsilon^{1 / 8}
$$

Next we suppose that there exists a solution $U$ of $(\lambda I-L(\mathbf{h})) U=\hat{\Psi}_{k}(\mathbf{h})$ for $k=0, \ldots, N, \lambda \in \Sigma$ with $C \varepsilon^{1 / 8} \leq|\lambda| \leq M$ for sufficiently large $M$. By the same argument as above, we see

$$
\left\|U+\frac{1}{\lambda^{2}} \hat{\Phi}_{k}(\mathbf{h})-\frac{1}{\lambda} \hat{\Psi}_{k}(\mathbf{h})\right\|_{X^{1}} \leq C \nu
$$


Finally we have a resolvent estimate. For $W \in X, U$ is supposed to be a solution of $(\lambda I-L(\mathbf{h})) U=W$. By the same argument as above, we obtain

$$
\begin{aligned}
\| U- & \sum_{k=0}^{N}\left(-\frac{1}{\lambda^{2}}\left\langle W, \hat{\Phi}_{k}^{*}(\mathbf{h})\right\rangle+\frac{1}{\lambda}\left\langle W, \hat{\Psi}_{k}^{*}(\mathbf{h})\right\rangle\right) \hat{\Phi}_{k}(\mathbf{h}) \\
& -\sum_{k=0}^{N} \frac{1}{\lambda}\left\langle W, \hat{\Phi}_{k}^{*}(\mathbf{h})\right\rangle \hat{\Psi}_{k}(\mathbf{h})\left\|_{X^{1}} \leq C\right\| W \|_{X}
\end{aligned}
$$

for $\lambda \in \Sigma$ with $C \varepsilon^{1 / 8} \leq|\lambda| \leq M$. This inequality implies that any $\lambda \in \Sigma$ with $C \varepsilon^{1 / 8} \leq|\lambda| \leq M$ belongs to the resolvent set of $L(\mathbf{h})$, and derives a resolvent estimate (11). In other words, if $\lambda \in \Sigma$ is an eigenvalue of $L(\mathbf{h})$, then $|\lambda| \leq C \varepsilon^{1 / 8}$ holds true.

As shown in Lemma $2,-L\left(\mathbf{h}^{*}\right)$ is a sectorial operator, and the real parts of all spectra of $-L\left(\mathbf{h}^{*}\right)+I$ have strictly positive values. Then we can define a fractional space by $X^{\omega} \equiv D\left(\left(-L\left(\mathbf{h}^{*}\right)+I\right)^{\omega}\right)$ for $\omega \in[0,1)$, where $D\left(\left(-L\left(\mathbf{h}^{*}\right)+I\right)^{\omega}\right)$ is a domain of $\left(-L\left(\mathbf{h}^{*}\right)+I\right)^{\omega}$. We denote the norm of $X^{\omega}$ by $\|\cdot\|_{\omega}$. Hereafter we fix $\omega \in(3 / 4,1)$ such that $X^{\omega}$ is imbedded into $\mathbb{R}^{2 N+2} \times L^{2}(\mathbb{T})$, and $\mathbb{R}^{2 N+2} \times C^{\zeta}(\mathbb{T})$, as shown in the following lemma.

Lemma 3 Set $\omega \in(3 / 4,1)$ and let $\zeta$ be a positive constant with

$$
\frac{3}{4}+\frac{\zeta}{2}<\omega
$$

If $L$ is greater than or equal to $1, X^{\omega}$ is imbedded into $\mathbb{R}^{2 N+2} \times L^{2}(\mathbb{T})$, and $\mathbb{R}^{2 N+2} \times C^{\zeta}(\mathbb{T})$. More precisely, there is $C>0$ independent of $L$ such that

$$
\|U\|_{\mathbb{R}^{2 N+2} \times L^{2}(\mathbb{T})} \leq C\|U\|_{\omega}, \quad\|U\|_{\mathbb{R}^{2 N+2} \times C^{\zeta}(\mathbb{T})} \leq C\|U\|_{\omega}
$$

uniformly in $L \geq 1$ and $U \in X^{\omega}$.

Proof It is well-known that $H^{1}(\mathbb{T})$ is embedded into $C^{1 / 2}(\mathbb{T})$. More precisely, there is a constant $C>0$ independent of $L \geq 1$ such that any $u \in H^{1}(\mathbb{T})$ satisfies $\|u\|_{C^{1 / 2}(\mathbb{T})} \leq C\|u\|_{H^{1}(\mathbb{T})}$ because $\left|u\left(z_{1}\right)-u\left(z_{2}\right)\right| \leq\left|z_{1}-z_{2}\right|_{\mathbb{T}}^{1 / 2}\left\|u^{\prime}\right\|_{L^{2}(\mathbb{T})}$ for any $u \in C^{\infty}(\mathbb{T})$ and $z_{1}, z_{2} \in \mathbb{R}$, where $|\cdot|_{\mathbb{T}}$ is defined by $|z|_{\mathbb{T}}=\min _{n \in \mathbb{Z}}|z-n L|$ for $z \in \mathbb{R}$. The inequality above is what is called the Morrey's inequality.

Define $A_{0} \equiv-\partial^{2} / \partial z^{2}+I: H^{1}(\mathbb{T}) \rightarrow\left(H^{1}(\mathbb{T})\right)^{*}$. We denote a fractional operator and its domain of $A_{0}$ by $A_{0}^{\omega}$ and $D\left(A_{0}^{\omega}\right)$. It is easy to see $\|u\|_{L^{2}(\mathbb{T})}=$ $\left\|A_{0}^{1 / 2} u\right\|_{\left(H^{1}(\mathbb{T})\right)^{*}}$ for any $u \in D\left(A_{0}^{1 / 2}\right)$ by the Riez representation theorem. Hence it can be proved that $u \in D\left(A_{0}^{\omega}\right)$ can be imbedded into $L^{2}(\mathbb{T})$ and $C^{\zeta}(\mathbb{T})$, and satisfies

$$
\|u\|_{L^{2}(\mathbb{T})} \leq C\left\|A_{0}^{\omega} u\right\|_{\left(H^{1}(\mathbb{T})\right)^{*}}, \quad\|u\|_{C^{\zeta}(\mathbb{T})} \leq C\left\|A_{0}^{\omega} u\right\|_{\left(H^{1}(\mathbb{T})\right)^{*}}
$$

by standard embedding theorems (see Theorem 1.6.1 in [11]) if $\omega$ and $\zeta$ satisfies (15). Moreover, it is obvious that $\left\|\left(\lambda I+A_{0}\right)^{-1}\right\| \leq C /|\lambda+1|$ holds true for real $\lambda>-1$ by a standard argument. 
Set $A \equiv\left(I_{2 N+2}, A_{0}\right): X^{1} \rightarrow X$ and $B \equiv-L\left(\mathbf{h}^{*}\right)+I: X^{1} \rightarrow X$. Since it is easy to see that $\|u\|_{H^{1}(\mathbb{T})}=\left\|A_{0} u\right\|_{\left(H^{1}(\mathbb{T})\right) *}$ for $u \in H^{1}(\mathbb{T})$, it follows from Lemma 2 that any positive real number $\lambda$ belongs to the resolvent set of $B$ and satisfies

$$
\left\|(\lambda I+A)(\lambda I+B)^{-1}\right\| \leq C .
$$

Simple calculations give us

$$
(\lambda I+A)^{-1}=\left(\frac{1}{\lambda+1} I_{2 N+2},\left(\lambda I+A_{0}\right)^{-1}\right)
$$

for any $\lambda \geq 0$ so that we have $A^{\omega}=\left(I_{2 N+2}, A_{0}^{\omega}\right)$. Then, the Morrey's inequality proved above, Lemma 2 and (17) imply

$$
\left\|(A-B)(\lambda I+A)^{-1}\right\| \leq C .
$$

In addition, we have

$$
A^{-\omega}-B^{-\omega}=-\frac{\sin \pi \omega}{\pi} \int_{0}^{\infty} \lambda^{-\omega}(\lambda I+A)^{-1}(A-B)(\lambda I+B)^{-1} d \lambda .
$$

Thanks to (17)-(19) and Theorem 1.4.4 in [11], we have

$$
\left\|I-A^{\omega} B^{-\omega}\right\| \leq C \int_{0}^{\infty} \lambda^{-\omega} \frac{1}{|\lambda+1|^{1-\omega}} \frac{1}{|\lambda+1|^{1-\omega}} d \lambda \leq C .
$$

Hence we see $X^{\omega} \subset D\left(A^{\omega}\right)$ and there is $C>0$ independent of $L \geq 1$ such that $\left\|A^{\omega} U\right\|_{X} \leq C\|U\|_{\omega}$ uniformly in $U \in X^{\omega}$, which completes the proof of Lemma 3 owing to (17).

\section{Construction of a homeomorphism $\Pi(\mathrm{h})$}

In the previous section we have studied spectra and estimated resolvent operators of $\mathcal{L}_{\infty}^{\prime}\left(0,0, p ; c_{0}, \mu_{0}, s_{0}\right)$ and $L(\mathbf{h})$. Our goal in this section is to construct a homeomorphism $\Pi(\mathbf{h})$ by using projection maps defined by a Dunford integral for the resolvent operator of $L(\mathbf{h})$. Such an operator was introduced in the previous work [3]. However, the lack of regularity of several functions makes it impossible to construct $\Pi(\mathbf{h})$ by the same way as in [3]. Then we will define $\Pi(\mathbf{h})$ discretely.

In the following we prove several lemmas. Lemmas 4-9 are shown by almost the same way as in [3] and [7]. However, our linearized operator has the Dirac delta functions, which lack the regularity of various functions, while the authors in the previous works [3] and [7] only considered smooth functions. Throughout this section, we denote general constants by $C$ independent of $\lambda, L, h^{*}$ and $\mathbf{h}$.

Let $\Gamma \equiv\{\lambda \in \mathbb{C}|| \lambda \mid=\sigma\} \subset \Sigma$ be a positively-oriented circle enclosing $\lambda=0$, where $\sigma \in(0, \alpha / 2)$ is sufficiently small and fixed independently of $h^{*}$. The fact (i) in Lemma 2 implies that $\Gamma$ is included in the resolvent set of $L(\mathbf{h})$ in $\mathbf{h} \in H\left(h^{*}\right)$. We define

$$
Q(\mathbf{h}) \equiv \frac{1}{2 \pi i} \int_{\Gamma}(\lambda I-L(\mathbf{h}))^{-1} d \lambda
$$

and $E(\mathbf{h}) \equiv Q(\mathbf{h}) X$. Then we see that $E(\mathbf{h})$ is a $2(N+1)$-dimensional space spanned by a basis which consists of (generalized) eigenfunctions of $L(\mathbf{h})$ approximated by $\Phi, \Psi$. 
Lemma 4 Set $\Phi_{k}(\mathbf{h}) \equiv Q(\mathbf{h}) \hat{\Phi}_{k}(\mathbf{h})$ and $\Psi_{k}(\mathbf{h}) \equiv Q(\mathbf{h}) \hat{\Psi}_{k}(\mathbf{h})$. If $h^{*}$ is sufficiently large, then $\left\{\Phi_{k}(\mathbf{h}), \Psi_{k}(\mathbf{h})\right\}_{k=0}^{N}$ is a basis of $E(\mathbf{h})$ for $\mathbf{h} \in H\left(h^{*}\right)$. In addition, there is $C>0$ independent of $h^{*}$ such that $\left\|\Phi_{k}(\mathbf{h})-\hat{\Phi}_{k}(\mathbf{h})\right\|_{X^{1}} \leq C \varepsilon^{1 / 4}$ and $\left\|\Psi_{k}(\mathbf{h})-\hat{\Psi}_{k}(\mathbf{h})\right\|_{X^{1}} \leq C \varepsilon^{1 / 4}$ uniformly in $k=0, \ldots, N$ and $\mathbf{h} \in H\left(h^{*}\right)$.

Proof By the similar argument to the proof of Lemma 2, there is a constant $C$ independent of $\lambda, \sigma, L, h^{*}, \mathbf{h}$ such that $\left\|(\lambda I-L(\mathbf{h}))^{-1} \hat{\Phi}_{k}(\mathbf{h})-\hat{\Phi}_{k}(\mathbf{h}) / \lambda\right\|_{X^{1}} \leq C \varepsilon^{1 / 4}$ for $\lambda \in \Sigma$ with $|\lambda|=\sigma$, where $h^{*}$ is sufficiently large dependently on $\sigma$. Due to definition of $Q(\mathbf{h})$ by the Dunford integral, we see $\left\|Q(\mathbf{h}) \hat{\Phi}_{k}(\mathbf{h})-\hat{\Phi}_{k}(\mathbf{h})\right\|_{X^{1}} \leq$ $C \varepsilon^{1 / 4}$. Similarly, we have $\left\|Q(\mathbf{h}) \hat{\Psi}_{k}(\mathbf{h})-\hat{\Psi}_{k}(\mathbf{h})\right\|_{X^{1}} \leq C \varepsilon^{1 / 4}$. This result implies that the dimension of $E(\mathbf{h})$ is larger than or equal to $2(N+1)$.

Finally, we prove that it is equal to $2(N+1)$. Set $W \in E(\mathbf{h})$ and assume

$$
\left\langle W, \hat{\Phi}_{i}^{*}(\mathbf{h})\right\rangle=\left\langle W, \hat{\Psi}_{i}^{*}(\mathbf{h})\right\rangle=0
$$

for all $i=0, \ldots, N$. Let $U$ be a solution of $(\lambda I-L(\mathbf{h})) U=W$ for $\lambda \in \Sigma$ with $|\lambda|=\sigma$. By (14) and Lemma 2 , we have $\|U\|_{X} \leq\|U\|_{X^{1}} \leq C\|W\|_{X}$ for a constant $C$ independent of $\lambda, \sigma, L, h^{*}, \mathbf{h}, W$. Hence we have

$$
\|W\|_{X}=\|Q(\mathbf{h}) W\|_{X}=\left\|\frac{1}{2 \pi i} \int_{\Gamma}(\lambda I-L(\mathbf{h}))^{-1} W d \lambda\right\|_{X} \leq C \sigma\|W\|_{X},
$$

which implies $W=0$ because $\sigma$ is small. Therefore the dimension of $E(\mathbf{h})$ is equal to $2(N+1)$.

Remark 2 Lemma 4 implies that $L(\mathbf{h})$ has exactly $2(N+1)$ eigenvalues included in $\left\{\lambda \in \mathbb{C}|| \lambda \mid \leq C_{1} \varepsilon^{1 / 8}\right\}$ for any $\mathbf{h} \in H\left(h^{*}\right)$, where $C_{1}$ is a positive constant given independently of $\mathbf{h}$ in Lemma 2 . Multiple eigenvalues are repeated as many times as their multiplicity indicates. Moreover, other spectra of $L(\mathbf{h})$ are in $\mathbb{C} \backslash \Sigma$.

Actually, the functions $\Phi_{k}(\mathbf{h})$ and $\Psi_{k}(\mathbf{h})$ are differentiable, and their derivatives are Hölder continuous with respect to $\mathbf{h} \in H\left(h^{*}\right)$, which will be verified in a general setting.

Lemma 5 Assume $3 / 4<\omega<1$ and set $W \in X^{\omega}$ with $\|W\|_{\omega}=1$. Put $\Phi(\mathbf{h})=$ $Q(\mathbf{h}) W$. If $h^{*}$ is sufficiently large, $\Phi(\mathbf{h})$ is differentiable with respect to $\mathbf{h} \in H\left(h^{*}\right)$. In addition, there is a constant $C>0$ independent of $h^{*}$ such that $\|\Phi(\mathbf{h})\|_{X^{1}} \leq C$, $\left\|\partial(\Phi(\mathbf{h})) / \partial h_{j}\right\|_{\mathbb{R}^{2 N+2} \times L^{2}(\mathbb{T})} \leq C$,

$$
\begin{aligned}
\left\|\Phi\left(\mathbf{h}_{1}\right)-\Phi\left(\mathbf{h}_{2}\right)\right\|_{\mathbb{R}^{2 N+2} \times L^{2}(\mathbb{T})} & \leq C\left|\mathbf{h}_{1}-\mathbf{h}_{2}\right|, \\
\left\|\Phi\left(\mathbf{h}_{1}\right)-\Phi\left(\mathbf{h}_{2}\right)\right\|_{X^{1}} & \leq C\left|\mathbf{h}_{1}-\mathbf{h}_{2}\right|^{1 / 2} \\
\left\|\frac{\partial \Phi}{\partial h_{j}}\left(\mathbf{h}_{1}\right)-\frac{\partial \Phi}{\partial h_{j}}\left(\mathbf{h}_{2}\right)\right\|_{X} & \leq C\left|\mathbf{h}_{1}-\mathbf{h}_{2}\right|^{1 / 2}
\end{aligned}
$$

uniformly in $j=0, \ldots, N-1, \mathbf{h}, \mathbf{h}_{1}, \mathbf{h}_{2} \in H\left(h^{*}\right)$, and $W \in X^{\omega}$ with $\|W\|_{\omega}=1$.

Before describing the proof of Lemma 5, we first study $\hat{\Phi}_{k}(\mathbf{h}), \hat{\Psi}_{k}(\mathbf{h}), \hat{\Phi}_{k}^{*}(\mathbf{h})$, $\hat{\Psi}_{k}^{*}(\mathbf{h})$. 
Lemma 6 Let $\Phi$ be equal to $\hat{\Phi}_{k}(\mathbf{h}), \hat{\Psi}_{k}(\mathbf{h}), \hat{\Phi}_{k}^{*}(\mathbf{h})$ or $\hat{\Psi}_{k}^{*}(\mathbf{h})$ for $k=0, \ldots, N$. Then $\Phi(\mathbf{h})$ is differentiable with respect to $\mathbf{h}$ in $X$, and it holds true that $\|\Phi(\mathbf{h})\|_{X^{1}} \leq C$, $\left\|\partial[\Phi(\mathbf{h})] / \partial h_{j}\right\|_{\mathbb{R}^{2 N+2} \times L^{2}(\mathbb{T})} \leq C$,

$$
\begin{aligned}
& \left\|\Phi\left(\mathbf{h}_{1}\right)-\Phi\left(\mathbf{h}_{2}\right)\right\|_{\mathbb{R}^{2 N+2} \times L^{2}(\mathbb{T})} \leq C\left|\mathbf{h}_{1}-\mathbf{h}_{2}\right| \\
& \left\|\Phi\left(\mathbf{h}_{1}\right)-\Phi\left(\mathbf{h}_{2}\right)\right\|_{X^{1}} \leq C\left|\mathbf{h}_{1}-\mathbf{h}_{2}\right|^{1 / 2}, \quad\left\|\frac{\partial \Phi}{\partial h_{j}}\left(\mathbf{h}_{1}\right)-\frac{\partial \Phi}{\partial h_{j}}\left(\mathbf{h}_{2}\right)\right\|_{X} \leq C\left|\mathbf{h}_{1}-\mathbf{h}_{2}\right|
\end{aligned}
$$

uniformly in $j=0, \ldots, N-1$ and $\mathbf{h}, \mathbf{h}_{1}, \mathbf{h}_{2} \in H\left(h^{*}\right)$.

Proof We first claim that the delta function $\delta_{z}$ satisfies

$$
\left\|\delta_{z_{1}}-\delta_{z_{2}}\right\|_{\left(H^{1}(\mathbb{T})\right)^{*}} \leq\left|z_{1}-z_{2}\right|_{\mathbb{T}}^{1 / 2}
$$

by the Morrey's inequality in the proof of Lemma 3, where $|\cdot|_{\mathbb{T}}^{1 / 2}$ was defined in the proof of Lemma 3.

In the following, let $C>0$ be a general constant independent of $\mathbf{h}, \mathbf{h}_{1}, \mathbf{h}_{2}$. Let $\varphi(\mathbf{h})=[\varphi(\mathbf{h})](z)$ be a function $\bar{\phi}\left(z-\bar{z}_{k}\right), \bar{\psi}\left(z-\bar{z}_{k}\right), \overline{\phi^{*}}\left(z-\bar{z}_{k}\right)$, or $\overline{\psi^{*}}\left(z-\bar{z}_{k}\right)$. We define $F(\mathbf{h}) \equiv \varphi(\mathbf{h})^{\prime \prime}-c_{0} \varphi(\mathbf{h})^{\prime}-\alpha \varphi(\mathbf{h})$, where the prime ' denotes differentiation with respect to $z$. It is easy to see $\varphi(\mathbf{h}) \in H^{1}(\mathbb{T})$. Hence we have $\|F(\mathbf{h})\|_{\left(H^{1}(\mathbb{T})\right)^{*}} \leq$ $C$ and $\left\|F\left(\mathbf{h}_{1}\right)-F\left(\mathbf{h}_{2}\right)\right\|_{\left(H^{1}(\mathbb{T})\right)^{*}} \leq C\left|\mathbf{h}_{1}-\mathbf{h}_{2}\right|^{1 / 2}$ by $(20)$ so that $\|\varphi(\mathbf{h})\|_{H^{1}(\mathbb{T})} \leq C$ and $\left\|\varphi\left(\mathbf{h}_{1}\right)-\varphi\left(\mathbf{h}_{2}\right)\right\|_{H^{1}(\mathbb{T})} \leq C\left|\mathbf{h}_{1}-\mathbf{h}_{2}\right|^{1 / 2}$ uniformly in $\mathbf{h}, \mathbf{h}_{1}, \mathbf{h}_{2} \in H\left(h^{*}\right)$ from the inequalities above and (9). We note that $\left|\bar{z}_{i}\left(\mathbf{h}_{1}\right)-\bar{z}_{i}\left(\mathbf{h}_{2}\right)\right|_{\mathbb{T}} \leq C\left|\mathbf{h}_{1}-\mathbf{h}_{2}\right|$ uniformly in $i=0, \ldots, N$ and $\mathbf{h}_{1}, \mathbf{h}_{2} \in H\left(h^{*}\right)$. Moreover, it is easy to see $\| \varphi\left(\mathbf{h}_{1}\right)-$ $\varphi\left(\mathbf{h}_{2}\right) \|_{L^{2}(\mathbb{T})} \leq C\left|\mathbf{h}_{1}-\mathbf{h}_{2}\right|$ uniformly in $\mathbf{h}_{1}, \mathbf{h}_{2} \in H\left(h^{*}\right)$.

Finally, we study $\partial \varphi(\mathbf{h}) / \partial h_{j}$. In case of $j \geq k$, we have $\partial \varphi(\mathbf{h}) / \partial h_{j}=0$. On the other hand, in case of $j<k$, we have $\partial \varphi(\mathbf{h}) / \partial h_{j}=-\varphi(\mathbf{h})^{\prime}$ and then $\left|\left\langle\partial \varphi(\mathbf{h}) / \partial h_{j}, w\right\rangle_{H^{1}(\mathbb{T})}\right| \leq C\|w\|_{L^{2}(\mathbb{T})}$ for any $w \in H^{1}(\mathbb{T})$. In addition, thanks to $\left\langle\varphi(\mathbf{h})^{\prime}, w\right\rangle_{L^{2}(\mathbb{T})}=\left\langle\varphi(\mathbf{h}), w^{\prime}\right\rangle_{L^{2}(\mathbb{T})}$ for any $w \in H^{1}(\mathbb{T})$, we see

$$
\left|\left\langle\frac{\partial \varphi}{\partial h_{j}}\left(\mathbf{h}_{1}\right)-\frac{\partial \varphi}{\partial h_{j}}\left(\mathbf{h}_{2}\right), w\right\rangle_{H^{1}(\mathbb{T})}\right| \leq C\left|\mathbf{h}_{1}-\mathbf{h}_{2}\right|\|w\|_{H^{1}(\mathbb{T})} .
$$

Since $w$ is an arbitrary function in $H^{1}(\mathbb{T})$ in the inequalities above, we have

$$
\left\|\frac{\partial \varphi}{\partial h_{j}}(\mathbf{h})\right\|_{L^{2}(\mathbb{T})} \leq C, \quad\left\|\frac{\partial \varphi}{\partial h_{j}}\left(\mathbf{h}_{1}\right)-\frac{\partial \varphi}{\partial h_{j}}\left(\mathbf{h}_{2}\right)\right\|_{\left(H^{1}(\mathbb{T})\right)^{*}} \leq C\left|\mathbf{h}_{1}-\mathbf{h}_{2}\right| .
$$

From the definitions of $\hat{\Phi}_{k}(\mathbf{h}), \hat{\Psi}_{k}(\mathbf{h}), \hat{\Phi}_{k}^{*}(\mathbf{h})$ or $\hat{\Psi}_{k}^{*}(\mathbf{h})$ and the orthogonal conditions of $\Phi, \Psi, \Phi^{*}, \Psi^{*}$, the following lemma holds true, which can be proved easily. We omit the details of the proof.

Lemma 7 Let $\mathbf{F}(\mathbf{h})$ be either $L(\mathbf{h}) \hat{\Phi}_{k}(\mathbf{h}), L(\mathbf{h}) \hat{\Psi}_{k}(\mathbf{h})+\hat{\Phi}_{k}(\mathbf{h}), L(\mathbf{h})^{*} \hat{\Phi}_{k}^{*}(\mathbf{h})$ or $L(\mathbf{h})^{*} \hat{\Psi}_{k}^{*}(\mathbf{h})+\hat{\Phi}_{k}^{*}(\mathbf{h})$ for $k=0, \ldots, N$. There is a constant $C>0$ independent of $h^{*}$ such that if $h^{*}$ is sufficiently large, then

$$
\begin{aligned}
& \|\mathbf{F}(\mathbf{h})\|_{X^{1}} \leq C \sqrt{\varepsilon}, \quad\left\|\mathbf{F}\left(\mathbf{h}_{1}\right)-\mathbf{F}\left(\mathbf{h}_{2}\right)\right\|_{X^{1}} \leq C\left|\mathbf{h}_{1}-\mathbf{h}_{2}\right|, \\
& \left\|\frac{\partial \mathbf{F}}{\partial h_{j}}(\mathbf{h})\right\|_{X^{1}} \leq C \sqrt{\varepsilon}, \quad\left\|\frac{\partial \mathbf{F}}{\partial h_{j}}\left(\mathbf{h}_{1}\right)-\frac{\partial \mathbf{F}}{\partial h_{j}}\left(\mathbf{h}_{2}\right)\right\|_{X^{1}} \leq C\left|\mathbf{h}_{1}-\mathbf{h}_{2}\right|
\end{aligned}
$$

uniformly in $j=0, \ldots, N-1$ and $\mathbf{h}, \mathbf{h}_{1}, \mathbf{h}_{2} \in H\left(h^{*}\right)$. 
Now we are in a position to prove Lemma 5 . Here we easily see that there is a positive constant $C$ independent of $h^{*}$ such that

$$
\begin{array}{ll}
\left|\left\langle\hat{\Phi}_{i}(\mathbf{h}), \hat{\Phi}_{k}^{*}(\mathbf{h})\right\rangle\right| \leq C \sqrt{\varepsilon}, & \left|\left\langle\hat{\Phi}_{i}(\mathbf{h}), \hat{\Psi}_{k}^{*}(\mathbf{h})\right\rangle-\delta_{i k}\right| \leq C \sqrt{\varepsilon} \\
\left|\left\langle\hat{\Psi}_{i}(\mathbf{h}), \hat{\Phi}_{k}^{*}(\mathbf{h})\right\rangle-\delta_{i k}\right| \leq C \sqrt{\varepsilon}, & \left|\left\langle\hat{\Psi}_{i}(\mathbf{h}), \hat{\Psi}_{k}^{*}(\mathbf{h})\right\rangle\right| \leq C \sqrt{\varepsilon}
\end{array}
$$

uniformly in $i, k=0, \ldots, N$ and $\mathbf{h} \in H\left(h^{*}\right)$. In the following proof of Lemma 5 , we apply the inequalities (22).

Proof Fix $\lambda \in \Sigma$ with $|\lambda|=\sigma$. We denote the solution of $(\lambda I-L(\mathbf{h})) U=W$ by $U(\mathbf{h})={ }^{t}\left(\hat{z}_{0}, \hat{y}_{0}, \ldots, \hat{z}_{N}, \hat{y}_{N}, u\right)$, where $\hat{z}_{i}=\hat{z}_{i}(\mathbf{h}), \hat{y}_{i}=\hat{y}_{i}(\mathbf{h})$, and $u=u(\mathbf{h})$. Throughout the proof, we denote a general constant independent of $\lambda, h^{*}$ by $C$, which may depend on $\sigma$. Moreover, $C_{0}$ denotes a general constant independent of $\lambda, \sigma, h^{*}$.

(11) implies $\|U(\mathbf{h})\|_{X^{1}} \leq C$. We set $V(\mathbf{h})=U(\mathbf{h})+\sum_{i=0}^{N} \hat{z}_{i} \hat{\Phi}_{i}(\mathbf{h})$. From $(\lambda I-$ $L(\mathbf{h})) U(\mathbf{h})=W$, we have

$$
(\lambda I-L(\mathbf{h})) V(\mathbf{h})=W-\sum_{i=0}^{N} \hat{z}_{i}(\lambda I-L(\mathbf{h})) \hat{\Phi}_{i}(\mathbf{h}) \equiv \mathbf{F}(\mathbf{h}, \mathbf{z}(\mathbf{h})),
$$

where $\mathbf{z}(\mathbf{h}) \equiv{ }^{t}\left(\hat{z}_{0}, \ldots, \hat{z}_{N}\right)$. If $h^{*}$ is sufficiently large, the function $\mathbf{F}(\mathbf{h}, \mathbf{z})$ satisfies

$$
\left\|\mathbf{F}\left(\mathbf{h}_{1}, \mathbf{z}_{1}\right)-\mathbf{F}\left(\mathbf{h}_{2}, \mathbf{z}_{2}\right)\right\|_{\mathbb{R}^{2 N+2} \times L^{2}(\mathbb{T})} \leq C_{0} \sigma\left|\mathbf{z}_{1}-\mathbf{z}_{2}\right|+C\left(\left|\mathbf{z}_{1}\right|+\left|\mathbf{z}_{2}\right|\right)\left|\mathbf{h}_{1}-\mathbf{h}_{2}\right|
$$

uniformly in $\mathbf{h}_{1}, \mathbf{h}_{2} \in H\left(h^{*}\right)$ and $\mathbf{z}_{1}, \mathbf{z}_{2} \in \mathbb{R}^{N+1}$ due to Lemmas 6 , 7. We denote the $(2 N+3)$-th component of $\mathbf{F}(\mathbf{h}, \mathbf{z})$ by $F^{u}(\mathbf{h}, \mathbf{z})$.

We denote the $(2 N+3)$-th component of $V(\mathbf{h})$ by $v=v(\mathbf{h})$. Since all $(2 i+1)$-th components of $V(\mathbf{h})$ are 0 in $i=0, \ldots, N, v$ satisfies

$$
\lambda v-\left(v^{\prime \prime}-c_{0} v^{\prime}-\alpha v\right)=F^{u}(\mathbf{h}, \mathbf{z}(\mathbf{h})),
$$

and $\left\|v\left(\mathbf{h}_{1}\right)-v\left(\mathbf{h}_{2}\right)\right\|_{H^{2}(\mathbb{T})} \leq C_{0} \sigma\left|\mathbf{z}\left(\mathbf{h}_{1}\right)-\mathbf{z}\left(\mathbf{h}_{2}\right)\right|+C\left|\mathbf{h}_{1}-\mathbf{h}_{2}\right|$ because of (9) and (24). Thanks to $\lambda \hat{z}_{i}-\hat{y}_{i}=\omega_{i}^{z}$, we see $\left|\hat{y}_{i}\left(\mathbf{h}_{1}\right)-\hat{y}_{i}\left(\mathbf{h}_{2}\right)\right|=\sigma\left|\hat{z}_{i}\left(\mathbf{h}_{1}\right)-\hat{z}_{i}\left(\mathbf{h}_{2}\right)\right|$. As a result, we have

$$
\left\|V\left(\mathbf{h}_{1}\right)-V\left(\mathbf{h}_{2}\right)\right\|_{\mathbb{R}^{2 N+2} \times H^{2}(\mathbb{T})} \leq C_{0} \sigma\left|\mathbf{z}\left(\mathbf{h}_{1}\right)-\mathbf{z}\left(\mathbf{h}_{2}\right)\right|+C\left|\mathbf{h}_{1}-\mathbf{h}_{2}\right| .
$$

Next we estimate $\left\langle U(\mathbf{h}), \hat{\Phi}_{i}^{*}(\mathbf{h})\right\rangle$ and $\left\langle U(\mathbf{h}), \hat{\Psi}_{i}^{*}(\mathbf{h})\right\rangle$. Since $U(\mathbf{h})$ satisfies $(\lambda I-$ $L(\mathbf{h})) U(\mathbf{h})=W$, we have

$$
\begin{aligned}
& \lambda\left\langle U(\mathbf{h}), \hat{\Phi}_{i}^{*}(\mathbf{h})\right\rangle=\left\langle L(\mathbf{h}) U(\mathbf{h}), \hat{\Phi}_{i}^{*}(\mathbf{h})\right\rangle+\left\langle W, \hat{\Phi}_{i}^{*}(\mathbf{h})\right\rangle \\
& =\left\langle L(\mathbf{h})^{*} \hat{\Phi}_{i}^{*}(\mathbf{h}), U(\mathbf{h})\right\rangle+\left\langle W, \hat{\Phi}_{i}^{*}(\mathbf{h})\right\rangle .
\end{aligned}
$$

From Lemmas $3,6,7$, and due to $|\lambda|=\sigma$ and $W \in X^{\omega}$ for $\omega \in(3 / 4,1)$, we have

$$
\left|\left\langle U\left(\mathbf{h}_{1}\right), \hat{\Phi}_{i}^{*}\left(\mathbf{h}_{1}\right)\right\rangle-\left\langle U\left(\mathbf{h}_{2}\right), \hat{\Phi}_{i}^{*}\left(\mathbf{h}_{2}\right)\right\rangle\right| \leq C \sqrt{\varepsilon}\left\|U\left(\mathbf{h}_{1}\right)-U\left(\mathbf{h}_{2}\right)\right\|_{X}+C\left|\mathbf{h}_{1}-\mathbf{h}_{2}\right|,
$$


where $h^{*}$ is sufficiently large dependent on $\sigma$. Similarly, we have

$\left|\left\langle U\left(\mathbf{h}_{1}\right), \hat{\Psi}_{i}^{*}\left(\mathbf{h}_{1}\right)\right\rangle-\left\langle U\left(\mathbf{h}_{2}\right), \hat{\Psi}_{i}^{*}\left(\mathbf{h}_{2}\right)\right\rangle\right| \leq C \sqrt{\varepsilon}\left\|U\left(\mathbf{h}_{1}\right)-U\left(\mathbf{h}_{2}\right)\right\|_{X}+C\left|\mathbf{h}_{1}-\mathbf{h}_{2}\right|$.

From $U(\mathbf{h})=V(\mathbf{h})-\sum_{i=0}^{N} \hat{z}_{i} \hat{\Phi}_{i}(\mathbf{h})$, we have

$$
\left\langle U(\mathbf{h}), \hat{\Psi}_{k}^{*}(\mathbf{h})\right\rangle=\left\langle V(\mathbf{h}), \hat{\Psi}_{k}^{*}(\mathbf{h})\right\rangle-\sum_{i=0}^{N} \hat{z}_{i}\left\langle\hat{\Phi}_{i}(\mathbf{h}), \hat{\Psi}_{k}^{*}(\mathbf{h})\right\rangle .
$$

Here we define an $(N+1) \times(N+1)$ matrix $A(\mathbf{h})=\left(a_{i k}(\mathbf{h})\right)_{k, i}$ and an $(N+1)$ dimensional vector $\mathbf{b}(\mathbf{h})=\left(b_{k}(\mathbf{h})\right)_{k}$ by $a_{i k}(\mathbf{h})=\left\langle\hat{\Phi}_{i}(\mathbf{h}), \hat{\Psi}_{k}^{*}(\mathbf{h})\right\rangle$ and $b_{k}(\mathbf{h})=$ $\left\langle V(\mathbf{h})-U(\mathbf{h}), \hat{\Psi}_{k}^{*}(\mathbf{h})\right\rangle$. From Lemma $6,(22),(26)$ and $(28)$, it is easy to see that $A(\mathbf{h})$ and $b(\mathbf{h})$ are differentiable in $\mathbf{h} \in H\left(h^{*}\right)$, and $\|A(\mathbf{h})-I\| \leq C \sqrt{\varepsilon},|b(\mathbf{h})| \leq C$,

$$
\begin{gathered}
\left\|A\left(\mathbf{h}_{1}\right)-A\left(\mathbf{h}_{2}\right)\right\| \leq C\left|\mathbf{h}_{1}-\mathbf{h}_{2}\right|, \quad\left\|\frac{\partial A}{\partial h_{j}}\left(\mathbf{h}_{1}\right)-\frac{\partial A}{\partial h_{j}}\left(\mathbf{h}_{2}\right)\right\| \leq C\left|\mathbf{h}_{1}-\mathbf{h}_{2}\right|, \\
\left|b\left(\mathbf{h}_{1}\right)-b\left(\mathbf{h}_{2}\right)\right| \leq C\left|\mathbf{h}_{1}-\mathbf{h}_{2}\right|+C \sqrt{\varepsilon}\left\|U\left(\mathbf{h}_{1}\right)-U\left(\mathbf{h}_{2}\right)\right\|_{X}+C_{0} \sigma\left|\mathbf{z}\left(\mathbf{h}_{1}\right)-\mathbf{z}\left(\mathbf{h}_{2}\right)\right| .
\end{gathered}
$$

The first inequality implies that $A(\mathbf{h})$ has an inverse $A(\mathbf{h})^{-1}$ with $\left\|A(\mathbf{h})^{-1}\right\| \leq C_{0}$. Since we have $\mathbf{z}(\mathbf{h})=A(\mathbf{h})^{-1} \mathbf{b}(\mathbf{h})$, we see

$$
\left|\mathbf{z}\left(\mathbf{h}_{1}\right)-\mathbf{z}\left(\mathbf{h}_{2}\right)\right| \leq C\left|\mathbf{h}_{1}-\mathbf{h}_{2}\right|+C \sqrt{\varepsilon}\left\|U\left(\mathbf{h}_{1}\right)-U\left(\mathbf{h}_{2}\right)\right\|_{X}+C_{0} \sigma\left|\mathbf{z}\left(\mathbf{h}_{1}\right)-\mathbf{z}\left(\mathbf{h}_{2}\right)\right|,
$$

from which we have $\left|\mathbf{z}\left(\mathbf{h}_{1}\right)-\mathbf{z}\left(\mathbf{h}_{2}\right)\right| \leq C\left|\mathbf{h}_{1}-\mathbf{h}_{2}\right|+C \sqrt{\varepsilon}\left\|U\left(\mathbf{h}_{1}\right)-U\left(\mathbf{h}_{2}\right)\right\|_{X}$ because sufficiently small $\sigma$ can be given independently of $h^{*}$. Hence we have

$$
\begin{aligned}
\left\|U\left(\mathbf{h}_{1}\right)-U\left(\mathbf{h}_{2}\right)\right\|_{X} & \leq\left\|V\left(\mathbf{h}_{1}\right)-V\left(\mathbf{h}_{2}\right)\right\|_{X}+\sum_{i=0}^{N}\left\|\hat{z}_{i}\left(\mathbf{h}_{1}\right) \hat{\Phi}_{i}\left(\mathbf{h}_{1}\right)-\hat{z}_{i}\left(\mathbf{h}_{2}\right) \hat{\Phi}_{i}\left(\mathbf{h}_{2}\right)\right\|_{X} \\
& \leq C \sqrt{\varepsilon}\left\|U\left(\mathbf{h}_{1}\right)-U\left(\mathbf{h}_{2}\right)\right\|_{X}+C\left|\mathbf{h}_{1}-\mathbf{h}_{2}\right|,
\end{aligned}
$$

from which we have

$$
\left\|U\left(\mathbf{h}_{1}\right)-U\left(\mathbf{h}_{2}\right)\right\|_{X} \leq C\left|\mathbf{h}_{1}-\mathbf{h}_{2}\right|
$$

if $h^{*}$ is sufficiently large. From Lemma 6, (26) and (29), we also have

$$
\begin{aligned}
& \left\|U\left(\mathbf{h}_{1}\right)-U\left(\mathbf{h}_{2}\right)\right\|_{\mathbb{R}^{2 N+2} \times L^{2}(\mathbb{T})} \leq C\left|\mathbf{h}_{1}-\mathbf{h}_{2}\right|, \\
& \left\|U\left(\mathbf{h}_{1}\right)-U\left(\mathbf{h}_{2}\right)\right\|_{X^{1}} \leq C\left|\mathbf{h}_{1}-\mathbf{h}_{2}\right|^{1 / 2} .
\end{aligned}
$$

Finally we prove the Hölder continuity of $\partial U(\mathbf{h}) / \partial h_{j}$ for $j=0, \ldots, N-1$. The Lipschitz continuity of $U(\mathbf{h})$ implies that $U(\mathbf{h})$ is differentiable almost everywhere in $\mathbf{h} \in H\left(h^{*}\right)$. First of all we have

$$
\left|\frac{\partial \hat{y}_{k}}{\partial h_{j}}\right|=\sigma\left|\frac{\partial \hat{z}_{k}}{\partial h_{j}}\right|, \quad\left\|\frac{\partial V}{\partial h_{j}}(\mathbf{h})\right\|_{X^{1}} \leq C_{0} \sigma\left|\frac{\partial \mathbf{z}}{\partial h_{j}}(\mathbf{h})\right|+C
$$


for $j=0, \ldots, N-1, k=0, \ldots, N$ from (26) if $h^{*}$ is sufficiently large. In addition, from (23), we have

$$
\begin{aligned}
& \lambda \hat{z}_{k}\left\langle\hat{\Phi}_{k}(\mathbf{h}), \hat{\Psi}_{k}^{*}(\mathbf{h})\right\rangle \\
& =-\left\langle(\lambda I-L(\mathbf{h}))^{*} \hat{\Psi}_{k}^{*}(\mathbf{h}), V(\mathbf{h})\right\rangle+\sum_{i=0}^{N} \hat{z}_{i}\left\langle L(\mathbf{h}) \hat{\Phi}_{i}(\mathbf{h}), \hat{\Psi}_{k}^{*}(\mathbf{h})\right\rangle \\
& +\left\langle W, \hat{\Psi}_{k}^{*}(\mathbf{h})\right\rangle-\lambda \sum_{i \neq k} \hat{z}_{i}\left\langle\hat{\Phi}_{i}(\mathbf{h}), \hat{\Psi}_{k}^{*}(\mathbf{h})\right\rangle,
\end{aligned}
$$

from which we have

$$
\left|\frac{\partial \hat{z}_{k}}{\partial h_{j}}\right| \leq C_{0} \sigma\left|\frac{\partial \mathbf{z}}{\partial h_{j}}(\mathbf{h})\right|+C
$$

for $j=0, \ldots, N-1, k=0, \ldots, N$ by Lemmas $6,7,(22)$ and (30). Hence we have $\left|\partial \mathbf{z}(\mathbf{h}) / \partial h_{j}\right| \leq C$ because $\sigma$ is small. Then we see $\left\|\partial V(\mathbf{h}) / \partial h_{j}\right\|_{X^{1}} \leq C$.

From $(25), \partial V(\mathbf{h}) / h_{j}$ satisfies

$$
\left\|\frac{\partial V}{\partial h_{j}}\left(\mathbf{h}_{1}\right)-\frac{\partial V}{\partial h_{j}}\left(\mathbf{h}_{2}\right)\right\|_{X^{1}} \leq C_{0} \sigma\left|\frac{\partial \mathbf{z}}{\partial h_{j}}\left(\mathbf{h}_{1}\right)-\frac{\partial \mathbf{z}}{\partial h_{j}}\left(\mathbf{h}_{2}\right)\right|+C\left|\mathbf{h}_{1}-\mathbf{h}_{2}\right| .
$$

Thanks to Lemma 6 and $\partial \hat{\Psi}_{k}^{*} / \partial h_{j}=-\partial \hat{\Psi}_{k}^{*} / \partial z$, we note

$$
\left|\left\langle W, \frac{\partial \hat{\Psi}_{k}^{*}}{\partial h_{j}}\left(\mathbf{h}_{1}\right)\right\rangle-\left\langle W, \frac{\partial \hat{\Psi}_{k}^{*}}{\partial h_{j}}\left(\mathbf{h}_{2}\right)\right\rangle\right| \leq C\left|\mathbf{h}_{1}-\mathbf{h}_{2}\right|^{1 / 2} .
$$

Differentiating both sides of (31) by $h_{j}$, we have

$$
\left|\frac{\partial \hat{z}_{k}}{\partial h_{j}}\left(\mathbf{h}_{1}\right)-\frac{\partial \hat{z}_{k}}{\partial h_{j}}\left(\mathbf{h}_{2}\right)\right| \leq C_{0} \sigma\left|\frac{\partial \mathbf{z}}{\partial h_{j}}\left(\mathbf{h}_{1}\right)-\frac{\partial \mathbf{z}}{\partial h_{j}}\left(\mathbf{h}_{2}\right)\right|+C\left|\mathbf{h}_{1}-\mathbf{h}_{2}\right|^{1 / 2},
$$

which implies

$$
\begin{aligned}
\left|\frac{\partial \mathbf{z}}{\partial h_{j}}\left(\mathbf{h}_{1}\right)-\frac{\partial \mathbf{z}}{\partial h_{j}}\left(\mathbf{h}_{2}\right)\right| & \leq C\left|\mathbf{h}_{1}-\mathbf{h}_{2}\right|^{1 / 2}, \\
\left\|\frac{\partial V}{\partial h_{j}}\left(\mathbf{h}_{1}\right)-\frac{\partial V}{\partial h_{j}}\left(\mathbf{h}_{2}\right)\right\|_{X^{1}} & \leq C\left|\mathbf{h}_{1}-\mathbf{h}_{2}\right|^{1 / 2} .
\end{aligned}
$$

This completes the proof.

Thanks to the Riez-Schauder's theorem (see [15]) and Remark 2, $L(\mathbf{h})^{*}$ has exactly $2(N+1)$ eigenvalues close to 0 . We denote the associated eigenspace of $L(\mathbf{h})^{*}$ by $E(\mathbf{h})^{*} \equiv Q(\mathbf{h})^{*} X$, where

$$
Q(\mathbf{h})^{*} \equiv \frac{1}{2 \pi i} \int_{\Gamma}\left(\lambda I-L(\mathbf{h})^{*}\right)^{-1} d \lambda
$$

Note that $Q(\mathbf{h})^{*}$ corresponds to the adjoint operator of $Q(\mathbf{h})$, that is, it holds true that

$$
\left\langle Q(\mathbf{h}) \Phi, \Phi^{*}\right\rangle=\left\langle\Phi, Q(\mathbf{h})^{*} \Phi^{*}\right\rangle
$$

for $\Phi, \Phi^{*} \in X^{1}$. By the similar argument of Lemma 4, we can give a basis of $E(\mathbf{h})^{*}$, denoted by $\left\{\tilde{\Phi}_{k}^{*}(\mathbf{h}), \tilde{\Psi}_{k}^{*}(\mathbf{h})\right\}_{k=0}^{N}$ as in the following lemma. 
Lemma 8 Set $\tilde{\Phi}_{k}^{*}(\mathbf{h}) \equiv Q(\mathbf{h})^{*} \hat{\Phi}_{k}^{*}(\mathbf{h})$ and $\tilde{\Psi}_{k}^{*}(\mathbf{h}) \equiv Q(\mathbf{h})^{*} \hat{\Psi}_{k}^{*}(\mathbf{h})$. If $h^{*}$ is sufficiently large, then $\left\{\tilde{\Phi}_{k}^{*}(\mathbf{h}), \tilde{\Psi}_{k}^{*}(\mathbf{h})\right\}_{k=0}^{N}$ is a basis of $E(\mathbf{h})^{*}$. In addition, there is a constant $C>0$ independent of $h^{*}$ such that $\left\|\tilde{\Phi}_{k}^{*}(\mathbf{h})-\hat{\Phi}_{k}^{*}(\mathbf{h})\right\|_{X^{1}} \leq C \varepsilon^{1 / 4}$ and $\left\|\tilde{\Psi}_{k}^{*}(\mathbf{h})-\hat{\Psi}_{k}^{*}(\mathbf{h})\right\|_{X^{1}} \leq C \varepsilon^{1 / 4}$ uniformly in $k=0, \ldots, N$ and $\mathbf{h} \in H\left(h^{*}\right)$.

From Lemmas 3, 5 and (32), we can easily prove that $\tilde{\Phi}_{k}^{*}(\mathbf{h})$ and $\tilde{\Psi}_{k}^{*}(\mathbf{h})$ are differentiable, and their derivatives are Hölder continuous with respect to $\mathbf{h} \in$ $H\left(h^{*}\right)$. More precisely, for $\Phi^{*}(\mathbf{h})=\hat{\Phi}_{k}^{*}(\mathbf{h})$ or $\hat{\Psi}_{k}^{*}(\mathbf{h})(k=0, \ldots, N)$ and $W \in X^{\omega}$ with $\omega \in(3 / 4,1)$, we set $F(\mathbf{h}) \equiv\left\langle Q(\mathbf{h}) W, \Phi^{*}(\mathbf{h})\right\rangle$. Then, $F(\mathbf{h})$ is differentiable with respect to $\mathbf{h}$ and satisfies

$$
\begin{aligned}
& |F(\mathbf{h})| \leq C, \quad\left|F\left(\mathbf{h}_{1}\right)-F\left(\mathbf{h}_{2}\right)\right| \leq C\left|\mathbf{h}_{1}-\mathbf{h}_{2}\right| \\
& \left|\frac{\partial F}{\partial h_{j}}(\mathbf{h})\right| \leq C, \quad\left|\frac{\partial F}{\partial h_{j}}\left(\mathbf{h}_{1}\right)-\frac{\partial F}{\partial h_{j}}\left(\mathbf{h}_{2}\right)\right| \leq C\left|\mathbf{h}_{1}-\mathbf{h}_{2}\right|^{1 / 2}
\end{aligned}
$$

uniformly in $j=0, \ldots, N-1, \mathbf{h}, \mathbf{h}_{1}, \mathbf{h}_{2} \in H\left(h^{*}\right)$, and $W \in X^{\omega}$ for $3 / 4<\omega<1$ with $\|W\|_{\omega}=1$.

We set

$$
\Phi_{k}^{*}(\mathbf{h}) \equiv \sum_{j=0}^{N}\left(a_{j k} \tilde{\Phi}_{j}^{*}(\mathbf{h})+b_{j k} \tilde{\Psi}_{j}^{*}(\mathbf{h})\right), \quad \Psi_{k}^{*}(\mathbf{h}) \equiv \sum_{j=0}^{N}\left(c_{j k} \tilde{\Phi}_{j}^{*}(\mathbf{h})+d_{j k} \tilde{\Psi}_{j}^{*}(\mathbf{h})\right)
$$

for constants $a_{j k}, b_{j k}, c_{j k}, d_{j k}$ in $j, k=0, \ldots, N$. It is obvious that $\Phi_{k}^{*}(\mathbf{h})$ and $\Psi_{k}^{*}(\mathbf{h})$ are included in $E(\mathbf{h})^{*}$ because of their definitions in (34) by linear combination of $\left\{\tilde{\Phi}_{i}^{*}(\mathbf{h}), \tilde{\Psi}_{i}^{*}(\mathbf{h})\right\}$. By choosing appropriate constants, $\Phi_{i}(\mathbf{h}), \Psi_{i}(\mathbf{h}), \Phi_{i}^{*}(\mathbf{h})$ and $\Psi_{i}^{*}(\mathbf{h})$ satisfy orthogonal conditions given in the following lemma.

Lemma 9 If $h^{*}$ is sufficiently large, then there exist $a_{j k}, b_{j k}, c_{j k}$ and $d_{j k}$ for $j, k=0, \ldots, N$ in (34) such that $\Phi_{i}(\mathbf{h}), \Psi_{i}(\mathbf{h}), \Phi_{k}^{*}(\mathbf{h})$, and $\Psi_{k}^{*}(\mathbf{h})$ satisfy

$$
\begin{array}{rlrl}
\left\langle\Phi_{i}(\mathbf{h}), \Psi_{k}^{*}(\mathbf{h})\right\rangle & =\delta_{i k}, & \left\langle\Phi_{i}(\mathbf{h}), \Phi_{k}^{*}(\mathbf{h})\right\rangle=0 \\
\left\langle\Psi_{i}(\mathbf{h}), \Phi_{k}^{*}(\mathbf{h})\right\rangle=\delta_{i k}, & \left\langle\Psi_{i}(\mathbf{h}), \Psi_{k}^{*}(\mathbf{h})\right\rangle=0
\end{array}
$$

for any $i, k=0, \ldots, N$, where $\delta_{i k}$ denotes the Kronecker delta. In addition, there are positive constants $C_{1}, C_{2}$ independent of $h^{*}$ such that the functions $a_{j k}=$ $a_{j k}(\mathbf{h}), b_{j k}=b_{j k}(\mathbf{h}), c_{j k}=c_{j k}(\mathbf{h})$ and $d_{j k}=d_{j k}(\mathbf{h})$ satisfy the following conditions;

(i) It holds true that

$$
\begin{aligned}
& \left|a_{j k}(\mathbf{h})-\delta_{j k}\right| \leq C_{1} \varepsilon^{1 / 4}, \quad\left|b_{j k}(\mathbf{h})\right| \leq C_{1} \varepsilon^{1 / 4}, \\
& \left|c_{j k}(\mathbf{h})\right| \leq C_{1} \varepsilon^{1 / 4}, \quad\left|d_{j k}(\mathbf{h})-\delta_{j k}\right| \leq C_{1} \varepsilon^{1 / 4}
\end{aligned}
$$

uniformly in $j, k=0, \ldots, N$ and $\mathbf{h} \in H\left(h^{*}\right)$.

(ii) Let $\varphi(\mathbf{h})$ be either $a_{j k}(\mathbf{h}), b_{j k}(\mathbf{h}), c_{j k}(\mathbf{h})$ or $d_{j k}(\mathbf{h})$ for $j, k=0, \ldots, N$. Then $\varphi(\mathbf{h})$ is differentiable in $\mathbf{h} \in H\left(h^{*}\right)$ and satisfies $\left|\partial(\varphi(\mathbf{h})) / \partial h_{i}\right| \leq C_{2}$,

$$
\left|\varphi\left(\mathbf{h}_{1}\right)-\varphi\left(\mathbf{h}_{2}\right)\right| \leq C_{2}\left|\mathbf{h}_{1}-\mathbf{h}_{2}\right|, \quad\left|\frac{\partial \varphi}{\partial h_{i}}\left(\mathbf{h}_{1}\right)-\frac{\partial \varphi}{\partial h_{i}}\left(\mathbf{h}_{2}\right)\right| \leq C_{2}\left|\mathbf{h}_{1}-\mathbf{h}_{2}\right|^{1 / 2}
$$

uniformly in $i, j, k=0, \ldots, N$ and $\mathbf{h}, \mathbf{h}_{1}, \mathbf{h}_{2} \in H\left(h^{*}\right)$. 
Thanks to $\hat{\Phi}_{k}(\mathbf{h}), \hat{\Psi}_{k}(\mathbf{h}), \hat{\Phi}_{k}^{*}(\mathbf{h}), \hat{\Psi}_{k}^{*}(\mathbf{h}) \in X^{1}$, Lemmas 5,6 and (32) imply Lemma 9. So we omit the details of the proofs.

From the orthogonal conditions (35), we note that the projection map $Q(\mathbf{h})$ can be represented by $Q(\mathbf{h}) U=\sum_{i=0}^{N}\left(\left\langle U, \Psi_{i}^{*}(\mathbf{h})\right\rangle \Phi_{i}(\mathbf{h})+\left\langle U, \Phi_{i}^{*}(\mathbf{h})\right\rangle \Psi_{i}(\mathbf{h})\right)$. Set $R(\mathbf{h}) \equiv I-Q(\mathbf{h})$. Let $E^{\perp}(\mathbf{h}) \equiv R(\mathbf{h}) X$. Note that $E^{\perp}(\mathbf{h})$ can be characterized by

$$
E^{\perp}(\mathbf{h})=\left\{U \in X \mid\left\langle U, \Phi_{i}^{*}(\mathbf{h})\right\rangle=\left\langle U, \Psi_{i}^{*}(\mathbf{h})\right\rangle=0 \quad(i=0, \ldots, N)\right\} .
$$

It is easy to see $\|Q(\mathbf{h})\| \leq C$ and $\|R(\mathbf{h})\| \leq C$ uniformly in $\mathbf{h} \in H\left(h^{*}\right)$. Next we prove the Hölder continuity of the projection maps $Q(\mathbf{h}), R(\mathbf{h})$.

Lemma 10 There is a positive constant $C$ independent of $h^{*}$ such that if $h^{*}$ is sufficiently large, then $\left\|Q\left(\mathbf{h}_{1}\right)-Q\left(\mathbf{h}_{2}\right)\right\| \leq C\left|\mathbf{h}_{1}-\mathbf{h}_{2}\right|^{1 / 2}$ and $\left\|R\left(\mathbf{h}_{1}\right)-R\left(\mathbf{h}_{2}\right)\right\| \leq$ $C\left|\mathbf{h}_{1}-\mathbf{h}_{2}\right|^{1 / 2}$ uniformly in $\mathbf{h}_{1}, \mathbf{h}_{2} \in H\left(h^{*}\right)$.

Proof Thanks to (20) and the Morrey's inequality as in the proof of Lemma 3, we easily see

$$
\left\|\left(L\left(\mathbf{h}_{1}\right)-L\left(\mathbf{h}_{2}\right)\right) \Phi\right\|_{X} \leq C\left|\mathbf{h}_{1}-\mathbf{h}_{2}\right|^{1 / 2}\|\Phi\|_{X^{1}}
$$

uniformly in $\mathbf{h}_{1}, \mathbf{h}_{2} \in H\left(h^{*}\right)$ and $\Phi \in X^{1}$. From the definition of $Q(\mathbf{h})$ and Lemma 2, we have

$$
\begin{aligned}
& \left\|Q\left(\mathbf{h}_{1}\right) \Phi-Q\left(\mathbf{h}_{2}\right) \Phi\right\|_{X} \\
& =\left\|\int_{\Gamma}\left(\lambda I-L\left(\mathbf{h}_{1}\right)\right)^{-1}\left(L\left(\mathbf{h}_{1}\right)-L\left(\mathbf{h}_{2}\right)\right)\left(\lambda I-L\left(\mathbf{h}_{2}\right)\right)^{-1} \Phi d \lambda\right\|_{X} \\
& \leq C\left|\mathbf{h}_{1}-\mathbf{h}_{2}\right|^{1 / 2}\|\Phi\|_{X}
\end{aligned}
$$

uniformly in $\mathbf{h}_{1}, \mathbf{h}_{2} \in H\left(h^{*}\right)$ and $\Phi \in X$. Hence we conclude Lemma 10 .

Remark 3 As described in Lemma $10, Q(\mathbf{h})$ is Hölder continuous but may not be differentiable in general. On the other hand, Lemma 5 implies that $Q(\mathbf{h}) W$ is differentiable and its derivative is Hölder continuous in $\mathbf{h} \in H\left(h^{*}\right)$ by the regularity of $W \in X^{\omega}$ with $3 / 4<\omega<1$.

We define $\left.Q_{\mathbf{h}_{1}}\left(\mathbf{h}_{2}\right) \equiv Q\left(\mathbf{h}_{2}\right)\right|_{E\left(\mathbf{h}_{1}\right)}$ and $\left.R_{\mathbf{h}_{1}}\left(\mathbf{h}_{2}\right) \equiv R\left(\mathbf{h}_{2}\right)\right|_{E^{\perp}\left(\mathbf{h}_{1}\right)}$. Then $Q_{\mathbf{h}_{1}}\left(\mathbf{h}_{2}\right)$ and $R_{\mathbf{h}_{1}}\left(\mathbf{h}_{2}\right)$ satisfy the following lemma.

Lemma 11 There exists $\eta>0$ independent of $h^{*}$ such that if $h^{*}$ is sufficiently large, then $Q_{\mathbf{h}_{1}}\left(\mathbf{h}_{2}\right): E\left(\mathbf{h}_{1}\right) \rightarrow E\left(\mathbf{h}_{2}\right)$ and $R_{\mathbf{h}_{1}}\left(\mathbf{h}_{2}\right): E^{\perp}\left(\mathbf{h}_{1}\right) \rightarrow E^{\perp}\left(\mathbf{h}_{2}\right)$ are homeomorphic for any $\mathbf{h}_{1}, \mathbf{h}_{2} \in H\left(h^{*}\right)$ with $\left|\mathbf{h}_{1}-\mathbf{h}_{2}\right| \leq \eta$. In addition, there is $C>0$ independent of $h^{*}$ such that the invertible operators satisfy $\left\|Q_{\mathbf{h}_{1}}\left(\mathbf{h}_{2}\right)^{-1}\right\| \leq$ $C$ and $\left\|R_{\mathbf{h}_{1}}\left(\mathbf{h}_{2}\right)^{-1}\right\| \leq C$ uniformly in $\mathbf{h}_{1}, \mathbf{h}_{2} \in H\left(h^{*}\right)$ with $\left|\mathbf{h}_{1}-\mathbf{h}_{2}\right| \leq \eta$.

Proof It is obvious that $Q_{\mathbf{h}_{1}}\left(\mathbf{h}_{2}\right)$ is continuous. Next we put $Q_{\mathbf{h}_{1}}\left(\mathbf{h}_{2}\right) V=0$ for $V \in E\left(\mathbf{h}_{1}\right)$. Then we have $V=R\left(\mathbf{h}_{2}\right) V$. Due to Lemma 10, we have

$$
\|V\|_{X}=\left\|R\left(\mathbf{h}_{2}\right) V\right\|_{X}=\left\|\left(R\left(\mathbf{h}_{1}\right)-R\left(\mathbf{h}_{2}\right)\right) V\right\|_{X} \leq C\left|\mathbf{h}_{1}-\mathbf{h}_{2}\right|^{1 / 2}\|V\|_{X} \leq \frac{1}{2}\|V\|_{X} .
$$

Here we assume that $\eta$ is sufficiently small such that $C \sqrt{\eta} \leq 1 / 2$, which implies $V=0$. Note that $\eta$ can be given independently of $h^{*}$. 
Finally we see that $Q_{\mathbf{h}_{1}}\left(\mathbf{h}_{2}\right)$ is surjective. Set $K \equiv Q\left(\mathbf{h}_{2}\right) R\left(\mathbf{h}_{1}\right): E\left(\mathbf{h}_{2}\right) \rightarrow$ $E\left(\mathbf{h}_{2}\right)$. Here we prove $\|K\| \leq 1 / 2$ for $\mathbf{h}_{1}, \mathbf{h}_{2} \in H\left(h^{*}\right)$ with $\left|\mathbf{h}_{1}-\mathbf{h}_{2}\right| \leq \eta$. For any $V \in E\left(\mathbf{h}_{2}\right)$, we have

$$
\begin{aligned}
\left\|Q\left(\mathbf{h}_{2}\right) R\left(\mathbf{h}_{1}\right) V\right\|_{X} & =\left\|Q\left(\mathbf{h}_{2}\right)\left(R\left(\mathbf{h}_{1}\right)-R\left(\mathbf{h}_{2}\right)\right) V\right\|_{X} \\
& \leq C\left|\mathbf{h}_{1}-\mathbf{h}_{2}\right|^{1 / 2}\|V\|_{X} \leq \frac{1}{2}\|V\|_{X} .
\end{aligned}
$$

Then $(I-K): E\left(\mathbf{h}_{2}\right) \rightarrow E\left(\mathbf{h}_{2}\right)$ is invertible. Set $V \in E\left(\mathbf{h}_{2}\right)$ arbitrarily and $U \equiv(I-K)^{-1} V \in E\left(\mathbf{h}_{2}\right)$. Then we see

$$
U=Q\left(\mathbf{h}_{2}\right) U=Q_{\mathbf{h}_{1}}\left(\mathbf{h}_{2}\right) Q\left(\mathbf{h}_{1}\right) U+Q\left(\mathbf{h}_{2}\right) R\left(\mathbf{h}_{1}\right) U=Q_{\mathbf{h}_{1}}\left(\mathbf{h}_{2}\right) Q\left(\mathbf{h}_{1}\right) U+K U .
$$

Hence we have $V=Q_{\mathbf{h}_{1}}\left(\mathbf{h}_{2}\right) Q\left(\mathbf{h}_{1}\right)(I-K)^{-1} V$. Because of $V_{1} \equiv Q\left(\mathbf{h}_{1}\right)(I-$ $K)^{-1} V \in E\left(\mathbf{h}_{1}\right)$ and $V=Q_{\mathbf{h}_{1}}\left(\mathbf{h}_{2}\right) V_{1}$, we see that $Q_{\mathbf{h}_{1}}\left(\mathbf{h}_{2}\right)$ is surjective.

Next we estimate $Q_{\mathbf{h}_{1}}\left(\mathbf{h}_{2}\right)^{-1}$. Thanks to $\|K\| \leq 1 / 2$, we consider the Neumann series and estimate

$$
\left\|(I-K)^{-1}\right\|=\left\|\sum_{n=0}^{\infty} K^{n}\right\| \leq \sum_{n=0}^{\infty}\|K\|^{n} \leq \sum_{n=0}^{\infty} \frac{1}{2^{n}}=2
$$

As seen above, we have $Q_{\mathbf{h}_{1}}\left(\mathbf{h}_{2}\right)^{-1}=Q\left(\mathbf{h}_{1}\right)(I-K)^{-1}$, and then $\left\|Q_{\mathbf{h}_{1}}\left(\mathbf{h}_{2}\right)^{-1}\right\|$ $=\left\|Q\left(\mathbf{h}_{1}\right)(I-K)^{-1}\right\| \leq C$.

By the same argument as above, we also prove that $R_{\mathbf{h}_{1}}\left(\mathbf{h}_{2}\right)$ is homeomorphic, and estimate $R_{\mathbf{h}_{1}}\left(\mathbf{h}_{2}\right)^{-1}$ so that we conclude Lemma 11 .

It follows from Lemma 11 that projection spaces $E\left(\mathbf{h}_{1}\right)\left(E^{\perp}\left(\mathbf{h}_{1}\right)\right)$ and $E\left(\mathbf{h}_{2}\right)$ $\left(E^{\perp}\left(\mathbf{h}_{2}\right)\right)$ are homeomorphic in the case of $\left|\mathbf{h}_{1}-\mathbf{h}_{2}\right| \leq \eta$. Then we can construct homeomorphisms $\pi_{Q}(\mathbf{h}): E\left(\mathbf{h}^{*}\right) \rightarrow E(\mathbf{h})$ and $\pi_{R}(\mathbf{h}): E^{\perp}\left(\mathbf{h}^{*}\right) \rightarrow E^{\perp}(\mathbf{h})$ for any $\mathbf{h} \in H\left(h^{*}\right)$, respectively, as follows. Let $n$ be a positive integer which satisfies $1 / n \leq \eta$. If we assume (6), $n$ can be given independently of $h^{*}$. For $\mathbf{h} \in H\left(h^{*}\right)$, we define $\mathbf{h}(k) \in H\left(h^{*}\right)$ by

$$
\mathbf{h}(k) \equiv\left(1-\frac{k}{n}\right) \mathbf{h}^{*}+\frac{k}{n} \mathbf{h}
$$

for $k=0,1, \ldots, n$. Then we set

$$
\pi_{Q}(\mathbf{h}) \equiv \prod_{k=1}^{n} Q_{\mathbf{h}(n-k)}(\mathbf{h}(n-k+1)), \quad \pi_{R}(\mathbf{h}) \equiv \prod_{k=1}^{n} R_{\mathbf{h}(n-k)}(\mathbf{h}(n-k+1))
$$

Since $Q_{\mathbf{h}(n-k)}(\mathbf{h}(n-k+1))$ and $R_{\mathbf{h}(n-k)}(\mathbf{h}(n-k+1))$ are homeomorphisms for any $k=1, \ldots, n$ due to $1 / n \leq \eta, \pi_{Q}(\mathbf{h}): E\left(\mathbf{h}^{*}\right) \rightarrow E(\mathbf{h})$ and $\pi_{R}(\mathbf{h}): E^{\perp}\left(\mathbf{h}^{*}\right) \rightarrow$ $E^{\perp}(\mathbf{h})$ are homeomorphisms in $\mathbf{h} \in H\left(h^{*}\right)$ from Lemma 11.

Using $\pi_{Q}(\mathbf{h}), \pi_{R}(\mathbf{h})$, we define an operator $\Pi(\mathbf{h}): X \rightarrow X$ by

$$
\Pi(\mathbf{h}) V \equiv \pi_{Q}(\mathbf{h}) Q\left(\mathbf{h}^{*}\right) V+\pi_{R}(\mathbf{h}) R\left(\mathbf{h}^{*}\right) V
$$

for $V \in X$. Then the operator $\Pi(\mathbf{h})$ satisfies the following lemma. 
Lemma 12 If $h^{*}$ is sufficiently large, $\Pi(\mathbf{h}): X \rightarrow X$ is homeomorphic and invertible for $\mathbf{h} \in H\left(h^{*}\right)$. Its inverse operator, denoted by $\Pi(\mathbf{h})^{-1}$, is explicitly given by

$$
\Pi(\mathbf{h})^{-1} V=\left(\pi_{Q}(\mathbf{h})\right)^{-1} Q(\mathbf{h}) V+\left(\pi_{R}(\mathbf{h})\right)^{-1} R(\mathbf{h}) V .
$$

In addition, there is a positive constant $C$ independent of $h^{*}$ such that

$$
\begin{array}{ll}
\|\Pi(\mathbf{h})\| \leq C, & \left\|\Pi\left(\mathbf{h}_{1}\right)-\Pi\left(\mathbf{h}_{2}\right)\right\| \leq C\left|\mathbf{h}_{1}-\mathbf{h}_{2}\right|^{1 / 2}, \\
\left\|\Pi(\mathbf{h})^{-1}\right\| \leq C, & \left\|\Pi\left(\mathbf{h}_{1}\right)^{-1}-\Pi\left(\mathbf{h}_{2}\right)^{-1}\right\| \leq C\left|\mathbf{h}_{1}-\mathbf{h}_{2}\right|^{1 / 2}
\end{array}
$$

uniformly in $\mathbf{h}, \mathbf{h}_{1}, \mathbf{h}_{2} \in H\left(h^{*}\right)$.

Proof We first see that $\Pi(\mathbf{h})$ is bijective. We suppose that $\Pi(\mathbf{h}) V=0$ for $V \in X$. Then we have $\pi_{Q}(\mathbf{h}) Q\left(\mathbf{h}^{*}\right) V=0$ and $\pi_{R}(\mathbf{h}) R\left(\mathbf{h}^{*}\right) V=0$. Here we note $Q\left(\mathbf{h}^{*}\right) V \in$ $E\left(\mathbf{h}^{*}\right)$ and $R\left(\mathbf{h}^{*}\right) V \in E^{\perp}\left(\mathbf{h}^{*}\right)$. In addition, $\pi_{Q}(\mathbf{h})$ and $\pi_{R}(\mathbf{h})$ are homeomorphisms so that $Q\left(\mathbf{h}^{*}\right) V=0$ and $R\left(\mathbf{h}^{*}\right) V=0$, which implies $V=0$. Set $V \in X$ arbitrarily. Putting $V_{Q}=\pi_{Q}(\mathbf{h})^{-1} Q(\mathbf{h}) V \in E\left(\mathbf{h}^{*}\right), V_{R}=\pi_{R}(\mathbf{h})^{-1} R(\mathbf{h}) V \in E^{\perp}\left(\mathbf{h}^{*}\right)$ and $\tilde{V}=V_{Q}+V_{R} \in X$, we have

$$
V=\pi_{Q}(\mathbf{h}) V_{Q}+\pi_{R}(\mathbf{h}) V_{R}=\pi_{Q}(\mathbf{h}) Q\left(\mathbf{h}^{*}\right) \tilde{V}+\pi_{R}(\mathbf{h}) R\left(\mathbf{h}^{*}\right) \tilde{V}=\Pi(\mathbf{h}) \tilde{V} .
$$

Hence $\Pi(\mathbf{h})$ is bijective. The equality above also implies (38).

Obviously, $\|\Pi(\mathbf{h})\| \leq C$ holds true by its definition. Since Lemma 11 implies $\left\|\pi_{Q}(\mathbf{h})^{-1}\right\| \leq C$ and $\left\|\pi_{R}(\mathbf{h})^{-1}\right\| \leq C$, we have $\left\|\Pi(\mathbf{h})^{-1}\right\| \leq\left\|\pi_{Q}(\mathbf{h})^{-1} Q(\mathbf{h})\right\|+$ $\left\|\pi_{R}(\mathbf{h})^{-1} R(\mathbf{h})\right\| \leq C$. Finally we prove the Hölder continuity of $\Pi(\mathbf{h})$ and $\Pi(\mathbf{h})^{-1}$. Let $\eta$ be given in Lemma 11. Then it suffices to consider the case of $\left|\mathbf{h}_{1}-\mathbf{h}_{2}\right| \leq \eta$. Define

$$
\mathbf{h}_{j}(k)=\left(1-\frac{k}{n}\right) \mathbf{h}^{*}+\frac{k}{n} \mathbf{h}_{j}
$$

for $j=1,2$ and $k=0, \ldots, n$. We have $\left|\mathbf{h}_{1}(k)-\mathbf{h}_{2}(k)\right| \leq \eta$ for any $k=0,1, \ldots, n$. Simple calculations give us

$$
\begin{aligned}
& \pi_{Q}\left(\mathbf{h}_{1}\right)-\pi_{Q}\left(\mathbf{h}_{2}\right)=\prod_{k=1}^{n} Q\left(\mathbf{h}_{1}(n-k+1)\right)-\prod_{k=1}^{n} Q\left(\mathbf{h}_{2}(n-k+1)\right) \\
& =\sum_{i=1}^{n}\left(\prod_{k=1}^{n-i} Q\left(\mathbf{h}_{2}(n-k+1)\right)\right) \\
& \cdot\left(Q\left(\mathbf{h}_{1}(i)\right)-Q\left(\mathbf{h}_{2}(i)\right)\right)\left(\prod_{k=n-i+2}^{n} Q\left(\mathbf{h}_{1}(n-k+1)\right)\right),
\end{aligned}
$$

where we denote $\prod_{k=1}^{0} Q\left(\mathbf{h}_{2}(n-k+1)\right)=I$ and $\prod_{k=n+1}^{n} Q\left(\mathbf{h}_{1}(n-k+1)\right)=I$. Therefore it follows from Lemma 10 that

$$
\left\|\pi_{Q}\left(\mathbf{h}_{1}\right)-\pi_{Q}\left(\mathbf{h}_{2}\right)\right\| \leq C \sum_{i=1}^{n}\left|\mathbf{h}_{1}(i)-\mathbf{h}_{2}(i)\right|^{1 / 2} \leq C\left|\mathbf{h}_{1}-\mathbf{h}_{2}\right|^{1 / 2} .
$$

Similarly, we have $\left\|\pi_{R}\left(\mathbf{h}_{1}\right)-\pi_{R}\left(\mathbf{h}_{2}\right)\right\| \leq C\left|\mathbf{h}_{1}-\mathbf{h}_{2}\right|^{1 / 2}$, from which we complete the proof of the lemma. 
Remark 4 It follows that $\left.\Pi(\mathbf{h})\right|_{E\left(\mathbf{h}^{*}\right)}=\pi_{Q}(\mathbf{h})$ and $\left.\Pi(\mathbf{h})\right|_{E^{\perp}\left(\mathbf{h}^{*}\right)}=\pi_{R}(\mathbf{h})$. In addition, there is $C>0$ independent of $h^{*}$ such that $\|Q(\mathbf{h}) U\|_{X^{1}} \leq C\|U\|_{X^{1}}$ and $\|R(\mathbf{h}) U\|_{X^{1}} \leq C\|U\|_{X^{1}}$ uniformly in $\mathbf{h} \in H\left(h^{*}\right)$ and $U \in X^{1}$. Moreover, it is easy to see $\Pi(\mathbf{h}) W \in X^{\omega}$ and $\|\Pi(\mathbf{h}) W\|_{\omega} \leq C\|W\|_{\omega}$ uniformly in $\mathbf{h} \in H\left(h^{*}\right)$ and $W \in X^{\omega}$ with $3 / 4<\omega<1$ for $C>0$ independent of $h^{*}$.

Remark 5 In order that $n$ is independent of $h^{*}$, the condition (6) is necessary. When all particles are positioned with almost the same distance between two neighboring particles in the circuit $\mathbb{T}$ for large $L,(6)$ does hold true.

\section{Definition of an evolution operator $T\left(t, t_{0} ; \mathrm{h}\right)$}

In this section we estimate the resolvent operator of $\tilde{L}(\mathbf{h}) \equiv \Pi(\mathbf{h})^{-1} L(\mathbf{h}) \Pi(\mathbf{h})$ and its continuity with respect to $\mathbf{h} \in H\left(h^{*}\right)$. As a result, we see that $\tilde{L}(\mathbf{h})$ generates an evolution operator $T\left(t, t_{0} ; \mathbf{h}\right)$.

Lemma 13 Set $\omega \in(3 / 4,1)$ and suppose that $h^{*}$ is sufficiently large. Then, there is a positive constant $C$ independent of $h^{*}$ such that the following inequalities hold true uniformly in $\mathbf{h}, \mathbf{h}_{1}, \mathbf{h}_{2} \in H\left(h^{*}\right)$ and $V \in X^{\omega}$ with $\|V\|_{\omega}=1$;

$$
\begin{gathered}
\left\|L(\mathbf{h}) Q\left(\mathbf{h}_{1}\right)\right\| \leq C, \quad\left\|L(\mathbf{h})\left(Q\left(\mathbf{h}_{1}\right)-Q\left(\mathbf{h}_{2}\right)\right)\right\| \leq C\left|\mathbf{h}_{1}-\mathbf{h}_{2}\right|^{\zeta}, \\
\left\|Q\left(\mathbf{h}_{1}\right) L(\mathbf{h}) V\right\|_{X} \leq C, \quad\left\|\left(Q\left(\mathbf{h}_{1}\right)-Q\left(\mathbf{h}_{2}\right)\right) L(\mathbf{h}) V\right\|_{X} \leq C\left|\mathbf{h}_{1}-\mathbf{h}_{2}\right|^{\zeta},
\end{gathered}
$$

where $\zeta$ is a positive constant satisfying (15).

Proof Throughout this proof, we denote general positive constants by $C$ independent of $h^{*}, \lambda, V, \mathbf{h}, \mathbf{h}_{1}, \mathbf{h}_{2}$. We have

$$
L(\mathbf{h}) Q\left(\mathbf{h}_{1}\right) V=\frac{1}{2 \pi i} \int_{\Gamma} L(\mathbf{h})\left(\lambda I-L\left(\mathbf{h}_{1}\right)\right)^{-1} V d \lambda
$$

for $V \in X$. Due to Lemma 2, we see $\left\|L(\mathbf{h})\left(\lambda I-L\left(\mathbf{h}_{1}\right)\right)^{-1} V\right\|_{X} \leq C\|V\|_{X} \leq C\|V\|_{\omega}$ uniformly in $\lambda \in \Gamma$ so that $\left\|L(\mathbf{h}) Q\left(\mathbf{h}_{1}\right) V\right\|_{X} \leq C\|V\|_{\omega}$. Similarly, we have

$$
\begin{aligned}
& L(\mathbf{h})\left(Q\left(\mathbf{h}_{1}\right)-Q\left(\mathbf{h}_{2}\right)\right) V \\
& =-\frac{1}{2 \pi i} \int_{\Gamma} L(\mathbf{h})\left(\lambda I-L\left(\mathbf{h}_{1}\right)\right)^{-1}\left(L\left(\mathbf{h}_{1}\right)-L\left(\mathbf{h}_{2}\right)\right)\left(\lambda I-L\left(\mathbf{h}_{2}\right)\right)^{-1} V d \lambda .
\end{aligned}
$$

Therefore we have $\left\|L(\mathbf{h})\left(Q\left(\mathbf{h}_{1}\right)-Q\left(\mathbf{h}_{2}\right)\right) V\right\|_{X} \leq C\left|\mathbf{h}_{1}-\mathbf{h}_{2}\right|^{1 / 2}\|V\|_{X}$ from (36). Here we note that $L(\mathbf{h}) Q\left(\mathbf{h}_{1}\right) V$ is well-defined because of $Q\left(\mathbf{h}_{1}\right) V \in X^{1}$ even for $V \in X$. that

It is easy to verify that there is a positive constant $C$ independent of $h^{*}$ such

$$
\left\|\left(L\left(\mathbf{h}_{1}\right)-L\left(\mathbf{h}_{2}\right)\right) V\right\|_{X} \leq C\left|\mathbf{h}_{1}-\mathbf{h}_{2}\right|^{\zeta}
$$

uniformly in $\mathbf{h}_{1}, \mathbf{h}_{2} \in H\left(h^{*}\right)$, and $V \in X^{\omega}$ with $\|V\|_{\omega}=1$ by Lemma 3 . Here we see $Q\left(\mathbf{h}_{1}\right) L(\mathbf{h})=L\left(\mathbf{h}_{1}\right) Q\left(\mathbf{h}_{1}\right)+Q\left(\mathbf{h}_{1}\right)\left(L(\mathbf{h})-L\left(\mathbf{h}_{1}\right)\right)$ so that the domain of $Q\left(\mathbf{h}_{1}\right) L(\mathbf{h})$ can be extended from $X^{1}$ to $X^{\omega}$. Therefore we see the first inequality in (40). By a similar argument, we can verify the second inequality of (40). 
Next we prove the following lemma.

Lemma 14 Set $\omega \in(3 / 4,1)$ and let $\zeta$ be a positive constant satisfying (15). Suppose that $h^{*}$ is sufficiently large. Then, there is a positive constant $C$ independent of $h^{*}$ such that

$$
\begin{array}{r}
\left\|L(\mathbf{h})\left(\Pi\left(\mathbf{h}_{1}\right)-\Pi\left(\mathbf{h}_{2}\right)\right) V\right\|_{X} \leq C\left|\mathbf{h}_{1}-\mathbf{h}_{2}\right|^{\zeta}\|V\|_{\omega} \\
\left\|\left(\Pi\left(\mathbf{h}_{1}\right)^{-1}-\Pi\left(\mathbf{h}_{2}\right)^{-1}\right) L(\mathbf{h}) V\right\|_{X} \leq C\left|\mathbf{h}_{1}-\mathbf{h}_{2}\right|^{\zeta}\|V\|_{\omega}
\end{array}
$$

uniformly in $\mathbf{h}, \mathbf{h}_{1}, \mathbf{h}_{2} \in H\left(h^{*}\right)$ and $V \in X^{\omega}$.

Proof It is obvious that the first inequality holds true from the definition of $\Pi(\mathbf{h})$ and Lemma 13. In order to have the second inequality, we have

$$
\begin{aligned}
\left(\Pi\left(\mathbf{h}_{1}\right)^{-1}-\Pi\left(\mathbf{h}_{2}\right)^{-1}\right) L(\mathbf{h}) V \\
=-\Pi\left(\mathbf{h}_{1}\right)^{-1}\left(\Pi\left(\mathbf{h}_{1}\right)-\Pi\left(\mathbf{h}_{2}\right)\right) \Pi\left(\mathbf{h}_{2}\right)^{-1} L(\mathbf{h}) V \\
=-\Pi\left(\mathbf{h}_{1}\right)^{-1}\left(\pi_{Q}\left(\mathbf{h}_{1}\right)-\pi_{Q}\left(\mathbf{h}_{2}\right)\right) Q\left(\mathbf{h}^{*}\right) \Pi\left(\mathbf{h}_{2}\right)^{-1} L(\mathbf{h}) V \\
\quad-\Pi\left(\mathbf{h}_{1}\right)^{-1}\left(\pi_{R}\left(\mathbf{h}_{1}\right)-\pi_{R}\left(\mathbf{h}_{2}\right)\right) R\left(\mathbf{h}^{*}\right) \Pi\left(\mathbf{h}_{2}\right)^{-1} L(\mathbf{h}) V .
\end{aligned}
$$

Both terms in the right-hand sides can be represented by a finite multiplicity of $Q\left(\mathbf{h}_{j}(k)\right)$ for $j=1,2$ and $k=0,1, \ldots, n$, where $\mathbf{h}_{j}(k)$ was introduced in the proof of Lemma 12. Thanks to Lemma 13, we can conclude the lemma.

Next we estimate the resolvent operator of $\tilde{L}(\mathbf{h})$ and its continuity with respect to $\mathbf{h} \in H\left(h^{*}\right)$, which implies that $\tilde{L}(\mathbf{h})$ generates an evolution operator.

Lemma 15 Suppose that $h^{*}$ is sufficiently large. Then, there is a positive constant $C$ independent of $h^{*}$ such that $\tilde{L}(\mathbf{h}): X^{1} \cap E^{\perp}\left(\mathbf{h}^{*}\right) \rightarrow E^{\perp}\left(\mathbf{h}^{*}\right)$ satisfies the following conditions;

(i) It holds true that

$$
\left\|(\lambda I-\tilde{L}(\mathbf{h}))^{-1}\right\|_{X} \leq \frac{C}{|\lambda+\alpha / 2|}, \quad\left\|(\lambda I-\tilde{L}(\mathbf{h}))^{-1} U\right\|_{X^{1}} \leq C\|U\|_{X}
$$

uniformly in $U \in E^{\perp}\left(\mathbf{h}^{*}\right), \mathbf{h} \in H\left(h^{*}\right)$ and $\lambda \in \Sigma$. Moreover, $-\tilde{L}(\mathbf{h})$ is a sectorial operator.

(ii) Set $\omega \in(3 / 4,1)$ and let $\zeta$ be a positive constant with (15). It holds true that

$$
\left\|\left(\tilde{L}\left(\mathbf{h}_{1}\right)-\tilde{L}\left(\mathbf{h}_{2}\right)\right) V\right\|_{X} \leq C\left|\mathbf{h}_{1}-\mathbf{h}_{2}\right|^{\zeta}\|V\|_{\omega}
$$

uniformly in $\mathbf{h}_{1}, \mathbf{h}_{2} \in H\left(h^{*}\right)$ and $V \in X^{\omega} \cap E^{\perp}\left(\mathbf{h}^{*}\right)$.

Proof The condition (i) can be easily shown by Lemmas 2, 12 and the facts in Chapter III, Section 6 of [15]. On the other hand, the condition (ii) is shown by (41), Remark 4, and Lemma 14. So we complete the proof of the lemma.

From the previous lemma, we easily prove the following lemma by the same argument as in the proof of Theorem 1.4.4 in [11]. So we omit the proof of Lemma 16. Note that $L\left(\mathbf{h}^{*}\right) U=\tilde{L}\left(\mathbf{h}^{*}\right) U$ in $U \in X^{1} \cap E^{\perp}\left(\mathbf{h}^{*}\right)$. 
Lemma 16 Set $\omega \in(3 / 4,1)$ and suppose that $h^{*}$ is sufficiently large. Then, there is a constant $C>0$ independent of $h^{*}$ such that

$$
\begin{gathered}
\left\|\left(-L\left(\mathbf{h}^{*}\right)+I\right)^{\omega}(-\tilde{L}(\mathbf{h}))^{-\omega} U\right\|_{X} \leq C\|U\|_{X}, \\
\left\|(-\tilde{L}(\mathbf{h}))^{\omega}\left(-L\left(\mathbf{h}^{*}\right)+I\right)^{-\omega} U\right\|_{X} \leq C\|U\|_{X}
\end{gathered}
$$

uniformly in $\mathbf{h} \in H\left(h^{*}\right)$ and $U \in E^{\perp}\left(\mathbf{h}^{*}\right)$.

Give $t_{*}, t^{*}$ with $0 \leq t_{*}<t^{*}$ arbitrarily. Let $\mathbf{h}(t):\left[t_{*}, t^{*}\right] \rightarrow H\left(h^{*}\right)$ be a $C^{1}$ function such that $\sup _{t \in\left(t_{*}, t^{*}\right)}|d \mathbf{h} / d t| \leq C\left(\varepsilon^{1 / 2}+\left(r^{*}\right)^{2}+|\kappa|\right)$ for a constant $C>0$ independent of $h^{*}, r^{*}, \kappa, t_{*}, t^{*}, \mathbf{h}$. Then, $B(t) \equiv \tilde{L}\left(\mathbf{h}^{*}\right)-\tilde{L}(\mathbf{h}(t)): X^{\omega} \cap E^{\perp}\left(\mathbf{h}^{*}\right) \rightarrow$ $E^{\perp}\left(\mathbf{h}^{*}\right)$ is Hölder continuous in $t \in\left[t_{*}, t^{*}\right]$ from Lemma 15 because we have

$$
\begin{aligned}
\left\|\left(B\left(t_{2}\right)-B\left(t_{1}\right)\right) V\right\|_{X} & \leq C\left|\mathbf{h}\left(t_{2}\right)-\mathbf{h}\left(t_{1}\right)\right|^{\zeta}\|V\|_{\omega} \\
& \leq C\left(\varepsilon^{1 / 2}+\left(r^{*}\right)^{2}+|\kappa|\right)^{\zeta}|t-s|^{\zeta}\|V\|_{\omega}
\end{aligned}
$$

uniformly in $t_{1}, t_{2}$ with $t_{*} \leq t_{1} \leq t_{2} \leq t^{*}$, where $\zeta$ satisfies (15). Hence $\tilde{L}(\mathbf{h})$ generates an evolution operator $T\left(t, t_{0} ; \mathbf{h}\right)$ thanks to Theorem 7.4.2 in [11]. In addition, for any $\beta_{2} \in(0, \alpha / 2)$, there are sufficiently large $h^{*}$, sufficiently small $r^{*},|\kappa|$ and a positive constant $C$ independent of $h^{*}, r^{*}, \kappa, t_{*}, t^{*}, \mathbf{h}$ such that

$$
\begin{aligned}
& \left\|T\left(t, t_{0} ; \mathbf{h}\right) V\right\|_{\omega} \leq C \max \left\{1,\left(t-t_{0}\right)^{-\omega}\right\} e^{-\beta_{2}\left(t-t_{0}\right)}\|V\|_{X}, \quad V \in E^{\perp}\left(\mathbf{h}^{*}\right), \\
& \left\|T\left(t, t_{0} ; \mathbf{h}\right) V\right\|_{\omega} \leq C e^{-\beta_{2}\left(t-t_{0}\right)}\|V\|_{\omega}, \quad V \in X^{\omega} \cap E^{\perp}\left(\mathbf{h}^{*}\right)
\end{aligned}
$$

uniformly in $t, t_{0}$ with $t_{*} \leq t_{0} \leq t \leq t^{*}$.

\section{Derivation of a reduced system and proof of the main theorem}

Our goal in this section is to prove the main theorem and derive a reduced system for $(l, \mathbf{h}, \mathbf{r})$. To begin with, we state the existence of a solution $U(z, t)$ in $(4)$. We denote the initial value of (4) at $t=0$ by $U_{0}$ and assume $U_{0} \in X^{\omega}$ with $\omega \in$ $(3 / 4,1)$ throughout this section. Then it can be shown by a standard argument for analytical semigroups that the solution $U(z, t)$ of $(4)$ exists uniquely and globally in time. Moreover, it is also continuous in $X^{\omega}$ and differentiable in $X$ with respect to $t$. Actually, we can also prove that $U(z, t)$ is differentiable for $t>0$ in $X^{\omega}$ as seen in the following lemma.

Lemma 17 Set $\omega \in(3 / 4,1)$. Let $U=U(z, t)$ be a solution of (4) with the initial value $U_{0} \in X^{\omega}$ at $t=0$. Then $\partial U / \partial t$ exists for $t>0$ in $X^{\omega}$.

Proof We denote $U(z, t)={ }^{t}\left(z_{0}(t), y_{0}(t), \ldots, z_{N}(t), y_{N}(t), u(z, t)\right)$ and may represent $z_{i}=z_{i}(t), y_{i}=y_{i}(t)$ and $u=u(z, t)$ for simplicity. We fix $t^{*}>0$ arbitrarily and consider $\partial U / \partial t$ for $t \in\left(0, t^{*}\right)$. In the proof, we denote general constants independent of $\kappa, t$ by $C$.

As stated above, it is easy to see that $\partial U / \partial t$ exists and is continuous for $t>0$ in $X$ by a standard semigroup argument. It means that $z_{i}^{\prime}$ and $y_{i}^{\prime}$ exist and are continuous for $t>0$. Moreover, it follows from (4) and the assumption $U_{0} \in X^{\omega}$ 
that $z_{i}^{\prime}$ and $y_{i}^{\prime}$ satisfy $\left|z_{i}^{\prime}\right| \leq C$ and $\left|y_{i}^{\prime}\right| \leq C$ uniformly in $t \in\left(0, t^{*}\right)$. Moreover, $\|U\|_{\omega} \leq C$ uniformly in $t \in\left(0, t^{*}\right)$.

Set $F(z, t) \equiv \sum_{i=0}^{N} \bar{f}\left(z-z_{i}, s\right)$ for $s=s_{0}+\kappa_{2}$. Then we see that $F=F(z, t)$ is differentiable with respect to $t$, and satisfies

$$
\left\|\frac{\partial F}{\partial t}\right\|_{\left(H^{1}(\mathbb{T})\right)^{*}}=\left\|\sum_{i=0}^{N}\left(s \delta_{z_{i}-\rho}-(s-1) \delta_{z_{i}}-\delta_{z_{i}+\rho}\right) z_{i}^{\prime}\right\|_{\left(H^{1}(\mathbb{T})\right)^{*}} \leq C .
$$

Hereafter we omit the $z$-dependency of the functions and abbreviate $u(z, t)$ and $F(z, t)$ as $u(t)$ and $F(t)$. The third equation of (4) can be written into an integral form

$$
u(t)=T(t) u_{0}+\int_{0}^{t} T(a) F(t-a) d a,
$$

where $u_{0}$ denotes the $(2 N+3)$-th component of $U_{0}$. Here $T(t)$ is an analytic semigroup generated by $A_{0}$, which is an operator from $H^{1}(\mathbb{T})$ to $\left(H^{1}(\mathbb{T})\right)^{*}$ and defined by $A_{0} v \equiv \partial^{2} v / \partial z^{2}-c_{0} \partial v / \partial z-\alpha v$ for $v \in H^{1}(\mathbb{T})$. Differentiating the both sides of the equation above with respect to $t$, we have

$$
\frac{\partial u}{\partial t}=A_{0} T(t) u_{0}+T(t) F(0)+\int_{0}^{t} T(a) \frac{\partial F}{\partial t}(t-a) d a .
$$

Due to $U_{0} \in X^{\omega}, u_{0} \in D\left(\left(-A_{0}\right)^{\omega}\right)$ holds true. Hence it follows from the equation above and $\|F(0)\|_{\left(H^{1}(\mathbb{T})\right)^{*}} \leq C$ that

$$
\left\|\left(-A_{0}\right)^{\omega} \frac{\partial u}{\partial t}\right\|_{\left(H^{1}(\mathbb{T})\right)^{*}} \leq C \max \left(1, \frac{1}{t^{\omega}}, \frac{1}{t}\right) e^{-\beta t}+C
$$

for some $\beta>0$. Setting $A \equiv\left(I_{2 N+2},-A_{0}\right): X^{1} \rightarrow X$, we see that $A$ is a sectorial operator and satisfies $\left\|\left(-L\left(\mathbf{h}^{*}\right)+I\right)^{\omega} A^{-\omega}\right\| \leq C$ and $\left\|A^{\omega}\left(-L\left(\mathbf{h}^{*}\right)+I\right)^{-\omega}\right\| \leq C$ by the same argument as in the proof of Theorem 1.4.4 in [11]. From this fact and (45), we complete the proof of Lemma 17.

We summarize the remainder of this section. To begin with, we will prove that we can transform the solution $U(z, t)$ of (4) to $(l(t), \mathbf{h}(t), \mathbf{r}(t), W(z, t))$ as

$$
U(z, t)=S(z, l(t), \mathbf{h}(t), \mathbf{r}(t))+\Xi(l(t)) \Pi(\mathbf{h}(t)) W(z, t)
$$

(see Lemma 18). Here we define the shift operator $\Xi(l): X \rightarrow X$ by $\left\langle\Xi(l) W, \Phi^{*}\right\rangle_{X^{1}}$ $\equiv\left\langle W, \Phi^{*}(\cdot+l)\right\rangle_{X^{1}}$ for $W \in X$ and $\Phi^{*} \in X^{1}$. For simplicity, we may represent $(l, \mathbf{h}, \mathbf{r}, W)=(l(t), \mathbf{h}(t), \mathbf{r}(t), W(z, t))$. In order to prove our main result stated in Section 1 , we derive a governing system $(60)$ of $(l, \mathbf{h}, \mathbf{r}, W)$ and estimate a function $W$ by using the evolution operator $T\left(t, t_{0} ; \mathbf{h}\right)$ generated by $\tilde{L}(\mathbf{h})$, where $\tilde{L}(\mathbf{h})$ is thought of as an operator from $X^{1} \cap E^{\perp}\left(\mathbf{h}^{*}\right)$ to $E^{\perp}\left(\mathbf{h}^{*}\right)$ (see (43)). Unfortunately, we can only verify the Hölder continuity of the nonlinear terms in the system (60) (Lemmas 19-21). This fact enables us to prove the existence of a solution in the system though we cannot expect the uniqueness of a solution. Eventually, we obtain Theorem 1, in which we will state our main result precisely.

We show that the transformation above is valid in Lemma 18. Here we denote $B_{\eta} \equiv\left\{V \in X^{\omega} \mid\|V\|_{\omega}<\eta\right\}$ for some $\eta>0$. 
Lemma 18 Set $\omega \in(3 / 4,1)$. There exists a positive constant $C$ independent of $h^{*}, r^{*}, \eta$ such that if $h^{*}$ is sufficiently large, and $r^{*}$ and $\eta$ are sufficiently small, for any $\left(l_{0}, \mathbf{h}_{0}, \mathbf{r}_{0}, V\right) \in \mathbb{R} \times H\left(h^{*}\right) \times R\left(r^{*}\right) \times B_{\eta}$, there exists $(l, \mathbf{h}, \mathbf{r}, W) \in \mathbb{R} \times$ $H\left(\tilde{h}^{*}\right) \times R\left(\tilde{r}^{*}\right) \times\left(B_{C \eta} \cap E^{\perp}\left(\mathbf{h}^{*}\right)\right)$ uniquely such that

$$
\begin{gathered}
V+S\left(z, l_{0}, \mathbf{h}_{\mathbf{0}}, \mathbf{r}_{\mathbf{0}}\right)=S(z, l, \mathbf{h}, \mathbf{r})+\Xi(l) \Pi(\mathbf{h}) W \\
\left|l-l_{0}\right| \leq C \eta, \quad\left|\mathbf{h}-\mathbf{h}_{0}\right| \leq C \eta, \quad\left|\mathbf{r}-\mathbf{r}_{0}\right| \leq C \eta, \quad\|W\|_{\omega} \leq C \eta,
\end{gathered}
$$

where $\tilde{h}^{*} \equiv h^{*}-C \eta$ and $\tilde{r}^{*} \equiv r^{*}+C \eta$. In addition, there exist the partial derivatives of $(l, \mathbf{h}, \mathbf{r}, W)$ satisfying (46) with respect to $l_{0}, \mathbf{h}_{0}, \mathbf{r}_{0}, V$, and their derivatives depend on $l_{0}, \mathbf{h}_{0}, \mathbf{r}_{0}, V$ continuously.

Proof Throughout this proof, we denote general positive constants by $C$ independent of $h^{*}, r^{*}, \eta$. Since $\Pi(\mathbf{h})$ is homeomorphic from $E^{\perp}\left(\mathbf{h}^{*}\right)$ to $E^{\perp}(\mathbf{h})$ and $\Xi(l)^{-1}=\Xi(-l)$, it suffices to show $\Xi(-l)\left(V+S\left(\cdot, l_{0}, \mathbf{h}_{\mathbf{0}}, \mathbf{r}_{\mathbf{0}}\right)-S(z, l, \mathbf{h}, \mathbf{r})\right) \in$ $E^{\perp}(\mathbf{h})$. This condition is equivalent to

$$
\begin{aligned}
& 0=\left\langle\Xi(-l)\left(V+S\left(\cdot, l_{0}, \mathbf{h}_{\mathbf{0}}, \mathbf{r}_{\mathbf{0}}\right)-S(\cdot, l, \mathbf{h}, \mathbf{r})\right), \Psi_{i}^{*}(\mathbf{h})\right\rangle \\
& 0=\left\langle\Xi(-l)\left(V+S\left(\cdot, l_{0}, \mathbf{h}_{\mathbf{0}}, \mathbf{r}_{\mathbf{0}}\right)-S(\cdot, l, \mathbf{h}, \mathbf{r})\right), \Phi_{i}^{*}(\mathbf{h})\right\rangle
\end{aligned}
$$

for all $i=0, \ldots, N$. Thus we define $F$ by

$$
\begin{aligned}
F_{i}(l, \mathbf{h}, \mathbf{r}) & \equiv\left\langle\Xi(-l)\left(V+S\left(\cdot, l_{0}, \mathbf{h}_{\mathbf{0}}, \mathbf{r}_{\mathbf{0}}\right)-S(\cdot, l, \mathbf{h}, \mathbf{r})\right), \Psi_{i}^{*}(\mathbf{h})\right\rangle, \\
F_{N+1+i}(l, \mathbf{h}, \mathbf{r}) & \equiv\left\langle\Xi(-l)\left(V+S\left(\cdot, l_{0}, \mathbf{h}_{\mathbf{0}}, \mathbf{r}_{\mathbf{0}}\right)-S(\cdot, l, \mathbf{h}, \mathbf{r})\right), \Phi_{i}^{*}(\mathbf{h})\right\rangle, \\
F(l, \mathbf{h}, \mathbf{r}) & \equiv{ }^{t}\left(F_{0}(l, \mathbf{h}, \mathbf{r}), \ldots, F_{2 N+1}(l, \mathbf{h}, \mathbf{r})\right)
\end{aligned}
$$

for $i=0, \ldots, N$ and find a zero $(l, \mathbf{h}, \mathbf{r})$ of $F$ for each $\left(l_{0}, \mathbf{h}_{0}, \mathbf{r}_{0}, V\right)$ by a contraction mapping theorem. Note that $\left|F\left(l_{0}, \mathbf{h}_{0}, \mathbf{r}_{0}\right)\right| \leq C \eta$.

First of all, we calculate the derivative of $F(l, \mathbf{h}, \mathbf{r})$. For $i=0, \ldots, N$, direct calculations give us

$$
\frac{\partial F_{i}}{\partial l}=\left\langle\Xi(-l) \frac{\partial V}{\partial z}+\Xi(-l) \frac{\partial S}{\partial z}\left(\cdot, l_{0}, \mathbf{h}_{\mathbf{0}}, \mathbf{r}_{\mathbf{0}}\right)-\sum_{i=0}^{N} e_{2 i+1}, \Psi_{i}^{*}(\mathbf{h})\right\rangle .
$$

Similarly we calculate the derivative of $F_{N+1+i}$ for $i=0, \ldots, N$ with respect to $l$. Then we have

$$
\begin{aligned}
\left|\frac{\partial F_{i}}{\partial l}\left(l_{0}, \mathbf{h}_{\mathbf{0}}, \mathbf{r}_{\mathbf{0}}\right)-1\right| & \leq C\left(r^{*}+\varepsilon^{1 / 4}+\eta\right), \\
\left|\frac{\partial F_{N+1+i}}{\partial l}\left(l_{0}, \mathbf{h}_{\mathbf{0}}, \mathbf{r}_{\mathbf{0}}\right)\right| & \leq C\left(r^{*}+\varepsilon^{1 / 4}+\eta\right)
\end{aligned}
$$

by (33) and Lemmas 4, 8, and 9 . Similarly we have

$$
\begin{aligned}
\frac{\partial F_{i}}{\partial h_{k}} & =\left\langle\sum_{i=k+1}^{N}\left(-e_{2 i+1}+\bar{p}^{\prime}\left(z-\bar{z}_{i}\right) e_{2 N+3}+r_{i} \bar{\psi}^{\prime}\left(z-\bar{z}_{i}\right) e_{2 N+3}\right), \Psi_{i}^{*}(\mathbf{h})\right\rangle \\
& +\left\langle\Xi(-l)\left(V+S\left(\cdot, l_{0}, \mathbf{h}_{\mathbf{0}}, \mathbf{r}_{\mathbf{0}}\right)-S(\cdot, l, \mathbf{h}, \mathbf{r})\right), \frac{\partial \Psi_{i}^{*}(\mathbf{h})}{\partial h_{k}}\right\rangle \\
\frac{\partial F_{i}}{\partial r_{m}} & =-\left\langle\hat{\Psi}_{m}^{*}(\mathbf{h}), \Psi_{i}^{*}(\mathbf{h})\right\rangle=0
\end{aligned}
$$


for $i, m=0, \ldots, N$ and $k=0, \ldots, N-1$ by (32) so that we have

$$
\left|\frac{\partial F_{i}}{\partial h_{k}}\left(l_{0}, \mathbf{h}_{\mathbf{0}}, \mathbf{r}_{\mathbf{0}}\right)-\sum_{j=k+1}^{N} \delta_{j i}\right| \leq C\left(r^{*}+\varepsilon^{1 / 4}+\eta\right) .
$$

Similarly we have

$$
\left|\frac{\partial F_{N+1+i}}{\partial h_{k}}\left(l_{0}, \mathbf{h}_{\mathbf{0}}, \mathbf{r}_{\mathbf{0}}\right)\right| \leq C\left(r^{*}+\varepsilon^{1 / 4}+\eta\right), \quad \frac{\partial F_{N+1+i}}{\partial r_{m}}\left(l_{0}, \mathbf{h}_{\mathbf{0}}, \mathbf{r}_{\mathbf{0}}\right)=-\delta_{m i}
$$

for $i, m=0, \ldots, N$ and $k=0, \ldots, N-1$. Thus we find

$$
\left\|\frac{\partial F}{\partial(l, \mathbf{h}, \mathbf{r})}\left(l_{0}, \mathbf{h}_{\mathbf{0}}, \mathbf{r}_{\mathbf{0}}\right)-\left(\begin{array}{cc}
M & 0 \\
0 & -I_{N+1}
\end{array}\right)\right\| \leq C\left(r^{*}+\varepsilon^{1 / 4}+\eta\right) .
$$

Here 0 is the $(N+1) \times(N+1)$ zero matrix. In addition, $M=\left(m_{i k}\right)_{i, k}$ is an $(N+1) \times(N+1)$ lower triangular matrix defined by $m_{i k}=1$ if $i \geq k$ and 0 if $i<k(i, k=1, \ldots, N+1)$. Hence $(\partial F / \partial(l, \mathbf{h}, \mathbf{r}))\left(l_{0}, \mathbf{h}_{\mathbf{0}}, \mathbf{r}_{\mathbf{0}}\right)$ is invertible. In addition, if $h^{*}$ is sufficiently large and $r^{*}, \eta$ are sufficiently small, it holds true that

$$
\left\|\left(\frac{\partial F}{\partial(l, \mathbf{h}, \mathbf{r})}\left(l_{0}, \mathbf{h}_{0}, \mathbf{r}_{0}\right)\right)^{-1}\right\| \leq C
$$

uniformly in $\left(l_{0}, \mathbf{h}_{0}, \mathbf{r}_{0}, V\right) \in \mathbb{R} \times H\left(h^{*}\right) \times R\left(r^{*}\right) \times B_{\eta}$

Next we show that for any $\nu_{1}>0$, there is $\nu_{2}>0$ given independently of $h^{*}, r^{*}, \eta$ such that

$$
\left\|\frac{\partial F}{\partial(l, \mathbf{h}, \mathbf{r})}(l, \mathbf{h}, \mathbf{r})-\frac{\partial F}{\partial(l, \mathbf{h}, \mathbf{r})}\left(l_{0}, \mathbf{h}_{0}, \mathbf{r}_{0}\right)\right\| \leq \nu_{1}
$$

uniformly in $\left(l_{0}, \mathbf{h}_{0}, \mathbf{r}_{0}, V\right) \in \mathbb{R} \times H\left(h^{*}\right) \times R\left(r^{*}\right) \times B_{\eta}$, and $(l, \mathbf{h}, \mathbf{r}) \in D$, where $D$ is a closed set defined by $D \equiv\left\{(l, \mathbf{h}, \mathbf{r}) \in \mathbb{R}^{2 N+2}||(l, \mathbf{h}, \mathbf{r})-\left(l_{0}, \mathbf{h}_{0}, \mathbf{r}_{0}\right) \mid \leq \nu_{2}\right\}$. We easily see that

$$
\begin{aligned}
& \left|\left\langle\Xi(-l) \frac{\partial V}{\partial z}, \Psi_{i}^{*}(\mathbf{h})\right\rangle-\left\langle\Xi\left(-l_{0}\right) \frac{\partial V}{\partial z}, \Psi_{i}^{*}\left(\mathbf{h}_{0}\right)\right\rangle\right| \\
& \leq\left|\left\langle\Xi(-l) \frac{\partial V}{\partial z}-\Xi\left(-l_{0}\right) \frac{\partial V}{\partial z}, \Psi_{i}^{*}(\mathbf{h})\right\rangle\right|+\left|\left\langle\Xi\left(-l_{0}\right) \frac{\partial V}{\partial z}, \Psi_{i}^{*}(\mathbf{h})-\Psi_{i}^{*}\left(\mathbf{h}_{0}\right)\right\rangle\right| \\
& \leq C\|V\|_{\omega}\left(\left\|\psi^{*}\left(\cdot-l_{0}\right)-\psi^{*}(\cdot-l)\right\|_{H^{1}(\mathbb{R})}+\left|\mathbf{h}-\mathbf{h}_{0}\right|^{1 / 2}\right)
\end{aligned}
$$

uniformly in $\left(l_{0}, \mathbf{h}_{0}, \mathbf{r}_{0}, V\right) \in \mathbb{R} \times H\left(h^{*}\right) \times R\left(r^{*}\right) \times B_{\eta}$ and $(l, \mathbf{h}, \mathbf{r}) \in D$ due to Lemmas 3, 6 and 10, where $\zeta$ is a positive constant satisfying (15). Here $C$ is a positive constant independent of not only $h^{*}, r^{*}, \eta$ but also $\nu_{1}, \nu_{2}$. Since we can also estimate the remainder terms, we conclude (49).

In order to find a zero $(l, \mathbf{h}, \mathbf{r})$ of $F$, it is sufficient to obtain a fixed point of $G(l, \mathbf{h}, \mathbf{r}): D \rightarrow D$ defined by

$$
G(l, \mathbf{h}, \mathbf{r}) \equiv\left(l_{0}, \mathbf{h}_{0}, \mathbf{r}_{0}\right)-\left(\frac{\partial F}{\partial(l, \mathbf{h}, \mathbf{r})}\left(l_{0}, \mathbf{h}_{0}, \mathbf{r}_{0}\right)\right)^{-1}\left(F\left(l_{0}, \mathbf{h}_{0}, \mathbf{r}_{0}\right)+F_{1}(l, \mathbf{h}, \mathbf{r})\right),
$$


where

$$
\begin{aligned}
F_{1}(l, \mathbf{h}, \mathbf{r}) \equiv & F(l, \mathbf{h}, \mathbf{r})-F\left(l_{0}, \mathbf{h}_{0}, \mathbf{r}_{0}\right) \\
& -\left(\frac{\partial F}{\partial(l, \mathbf{h}, \mathbf{r})}\left(l_{0}, \mathbf{h}_{0}, \mathbf{r}_{0}\right)\right)\left(l-l_{0}, \mathbf{h}-\mathbf{h}_{0}, \mathbf{r}-\mathbf{r}_{0}\right)
\end{aligned}
$$

Since $F(l, \mathbf{h}, \mathbf{r})$ is differentiable and its derivative is continuous by the calculations above, we obtain

$$
\begin{aligned}
F(l, \mathbf{h}, \mathbf{r}) & =F\left(l_{0}, \mathbf{h}_{0}, \mathbf{r}_{0}\right) \\
& +\left(\int_{0}^{1} \frac{\partial F}{\partial(l, \mathbf{h}, \mathbf{r})}(l(a), \mathbf{h}(a), \mathbf{r}(a)) d a\right)\left(l-l_{0}, \mathbf{h}-\mathbf{h}_{0}, \mathbf{r}-\mathbf{r}_{0}\right)
\end{aligned}
$$

where $(l(a), \mathbf{h}(a), \mathbf{r}(a)) \equiv(1-a)\left(l_{0}, \mathbf{h}_{0}, \mathbf{r}_{0}\right)+a(l, \mathbf{h}, \mathbf{r})$ for $a \in[0,1]$. Then, due to (48) and (49), we see

$$
\begin{aligned}
& \left|G(l, \mathbf{h}, \mathbf{r})-\left(l_{0}, \mathbf{h}_{0}, \mathbf{r}_{0}\right)\right| \\
& \leq C\left|F\left(l_{0}, \mathbf{h}_{0}, \mathbf{r}_{0}\right)\right|+C \nu_{2} \int_{0}^{1}\left\|\frac{\partial F}{\partial(l, \mathbf{h}, \mathbf{r})}(l(a), \mathbf{h}(a), \mathbf{r}(a))-\frac{\partial F}{\partial(l, \mathbf{h}, \mathbf{r})}\left(l_{0}, \mathbf{h}_{0}, \mathbf{r}_{0}\right)\right\| d a \\
& \leq \nu_{2}
\end{aligned}
$$

uniformly in $\left(l_{0}, \mathbf{h}_{0}, \mathbf{r}_{0}, V\right) \in \mathbb{R} \times H\left(h^{*}\right) \times R\left(r^{*}\right) \times B_{\eta}$, and $(l, \mathbf{h}, \mathbf{r}) \in D$ if $\eta$ is sufficiently small for $\nu_{2}$ and $\nu_{1}$ is sufficiently small independently of $\eta$, where $C$ is a positive constant independent of $h^{*}, r^{*}, \eta, \nu_{1}, \nu_{2}$. Similarly, we have

$$
\left|G\left(l_{1}, \mathbf{h}_{1}, \mathbf{r}_{1}\right)-G\left(l_{2}, \mathbf{h}_{2}, \mathbf{r}_{2}\right)\right| \leq \frac{1}{2}\left|\left(l_{1}, \mathbf{h}_{1}, \mathbf{r}_{1}\right)-\left(l_{2}, \mathbf{h}_{2}, \mathbf{r}_{2}\right)\right|
$$

uniformly in $\left(l_{0}, \mathbf{h}_{0}, \mathbf{r}_{0}, V\right) \in \mathbb{R} \times H\left(h^{*}\right) \times R\left(r^{*}\right) \times B_{\eta},\left(l_{1}, \mathbf{h}_{1}, \mathbf{r}_{1}\right),\left(l_{2}, \mathbf{h}_{2}, \mathbf{r}_{2}\right) \in D$. Hence $G$ is a uniform contraction so that there is a unique fixed point of $G$, or equivalently a zero $(l, \mathbf{h}, \mathbf{r})$ of $F$ by the contraction mapping theorem.

It is easy to see that there exist the partial derivatives of $(l, \mathbf{h}, \mathbf{r})$ with respect to $\left(l_{0}, \mathbf{h}_{0}, \mathbf{r}_{0}, V\right)$, and their derivatives depend on $\left(l_{0}, \mathbf{h}_{0}, \mathbf{r}_{0}, V\right)$ continuously.

Using the notation in Lemma 18, we can decompose a solution $U(z, t)$ of (4) into $(l, \mathbf{h}, \mathbf{r})=(l(t), \mathbf{h}(t), \mathbf{r}(t)) \in \mathbb{R} \times H\left(h^{*}\right) \times R\left(r^{*}\right)$ and $W=W(z, t) \in B_{\eta} \cap E^{\perp}\left(\mathbf{h}^{*}\right)$. More precisely, we have the following corollary.

Corollary 1 Set $\omega \in(3 / 4,1)$. Suppose that $h^{*}$ is sufficiently large, and $r^{*}$ and $\eta$ are sufficiently small. For some $t_{*} \geq 0$, let $U(z, t)$ be continuous for $t \geq t_{*}$ and differentiable for $t>t_{*}$ in $X^{\omega}$ such that $U\left(z, t_{*}\right)=S\left(z, l_{0}, \mathbf{h}_{0}, \mathbf{r}_{0}\right)+\Xi\left(l_{0}\right) \Pi\left(\mathbf{h}_{\mathbf{0}}\right) W_{0}(z)$ for some $\left(l_{0}, \mathbf{h}_{0}, \mathbf{r}_{0}\right) \in \mathbb{R} \times H\left(h^{*}\right) \times R\left(r^{*}\right)$ and $W_{0} \in B_{\eta} \cap E^{\perp}\left(\mathbf{h}^{*}\right)$. Then, there exist $t^{*}>t_{*}$ and a continuous function $(l, \mathbf{h}, \mathbf{r}, W)=(l(t), \mathbf{h}(t), \mathbf{r}(t), W(z, t))$ in $\mathbb{R}^{2 N+2} \times X^{\omega}$ for $t \in\left[t_{*}, t^{*}\right)$ such that $(l, \mathbf{h}, \mathbf{r}) \in \mathbb{R} \times H\left(h^{*}\right) \times R\left(r^{*}\right), W \in$ $B_{\eta} \cap E^{\perp}\left(\mathbf{h}^{*}\right)$, and

$$
U(z, t)=S(z, l, \mathbf{h}, \mathbf{r})+\Xi(l) \Pi(\mathbf{h}) W(z, t)
$$

hold true in $t_{*} \leq t<t^{*}$, and $\left(l\left(t_{*}\right), \mathbf{h}\left(t_{*}\right), \mathbf{r}\left(t_{*}\right), W\left(z, t_{*}\right)\right)=\left(l_{0}, \mathbf{h}_{0}, \mathbf{r}_{0}, W_{0}\right)$. Moreover, $(l, \mathbf{h}, \mathbf{r}, W)$ is differentiable for $t \in\left(t_{*}, t^{*}\right)$ in $\mathbb{R}^{2 N+2} \times X^{\omega}$. 
Now we are in a position to derive governing equations of $l, \mathbf{h}, \mathbf{r}, W$. Substituting $U(z, t)=S(z, l, \mathbf{h}, \mathbf{r})+\Xi(l) \Pi(\mathbf{h}) W(z, t)$ into the system (4), we have

$$
\begin{aligned}
\frac{\partial U}{\partial t}= & \Xi(l)\left(\frac{\partial S}{\partial l}(z, 0, \mathbf{h}, \mathbf{r})-\frac{\partial}{\partial z} \Pi(\mathbf{h}) W\right) \frac{d l}{d t} \\
& +\Xi(l)\left(\frac{\partial S}{\partial \mathbf{h}}(z, 0, \mathbf{h}, \mathbf{r})+\frac{\partial}{\partial \mathbf{h}} \Pi(\mathbf{h}) W\right) \frac{d \mathbf{h}}{d t} \\
& +\Xi(l) \frac{\partial S}{\partial \mathbf{r}}(z, 0, \mathbf{h}, \mathbf{r}) \frac{d \mathbf{r}}{d t}+\Xi(l) \Pi(\mathbf{h}) \frac{\partial W}{\partial t}
\end{aligned}
$$

and

$$
\mathcal{L}(U, \kappa)=\mathcal{L}(S(z, l, \mathbf{h}, \mathbf{r})+\Xi(l) \Pi(\mathbf{h}) W, \kappa)=\Xi(l) \mathcal{L}(S(z, 0, \mathbf{h}, \mathbf{r})+\Pi(\mathbf{h}) W, \kappa) .
$$

Remark 4 and Lemma 5 imply that $\partial(\Pi(\mathbf{h}) W) / \partial z$ and $\partial(\Pi(\mathbf{h}) W) / \partial \mathbf{h}$ are welldefined. Hence it follows that

$$
\begin{aligned}
& \left(\frac{\partial S}{\partial l}(z, 0, \mathbf{h}, \mathbf{r})-\frac{\partial}{\partial z} \Pi(\mathbf{h}) W\right) \frac{d l}{d t}+\left(\frac{\partial S}{\partial \mathbf{h}}(z, 0, \mathbf{h}, \mathbf{r})+\frac{\partial}{\partial \mathbf{h}} \Pi(\mathbf{h}) W\right) \frac{d \mathbf{h}}{d t} \\
& +\frac{\partial S}{\partial \mathbf{r}}(z, 0, \mathbf{h}, \mathbf{r}) \frac{d \mathbf{r}}{d t}+\Pi(\mathbf{h}) \frac{\partial W}{\partial t}=\mathcal{L}(S(z, 0, \mathbf{h}, \mathbf{r})+\Pi(\mathbf{h}) W, \kappa)
\end{aligned}
$$

Multiplying $Q(\mathbf{h})$ and $R(\mathbf{h})$ to $(51)$, we decompose it into

$$
\begin{aligned}
Q(\mathbf{h}) & {\left[\left(\frac{\partial S}{\partial l}(z, 0, \mathbf{h}, \mathbf{r})-\frac{\partial}{\partial z} \Pi(\mathbf{h}) W\right) \frac{d l}{d t}\right.} \\
+ & \left.\left(\frac{\partial S}{\partial \mathbf{h}}(z, 0, \mathbf{h}, \mathbf{r})+\frac{\partial}{\partial \mathbf{h}} \Pi(\mathbf{h}) W\right) \frac{d \mathbf{h}}{d t}+\frac{\partial S}{\partial \mathbf{r}}(z, 0, \mathbf{h}, \mathbf{r}) \frac{d \mathbf{r}}{d t}\right] \\
= & Q(\mathbf{h}) \mathcal{L}(S(z, 0, \mathbf{h}, \mathbf{r})+\Pi(\mathbf{h}) W, \kappa)
\end{aligned}
$$

and

$$
\begin{aligned}
& \Pi^{-1}(\mathbf{h}) R(\mathbf{h})\left[\left(\frac{\partial S}{\partial l}(z, 0, \mathbf{h}, \mathbf{r})-\frac{\partial}{\partial z} \Pi(\mathbf{h}) W\right) \frac{d l}{d t}\right. \\
& \left.+\left(\frac{\partial S}{\partial \mathbf{h}}(z, 0, \mathbf{h}, \mathbf{r})+\frac{\partial}{\partial \mathbf{h}} \Pi(\mathbf{h}) W\right) \frac{d \mathbf{h}}{d t}+\frac{\partial S}{\partial \mathbf{r}}(z, 0, \mathbf{h}, \mathbf{r}) \frac{d \mathbf{r}}{d t}\right]+\frac{\partial W}{\partial t} \\
& =\Pi^{-1}(\mathbf{h}) R(\mathbf{h}) \mathcal{L}(S(z, 0, \mathbf{h}, \mathbf{r})+\Pi(\mathbf{h}) W, \kappa) .
\end{aligned}
$$

The left-hand side of (52) can be represented as a linear combination of $d l / d t$, $d \mathbf{h} / d t$ and $d \mathbf{r} / d t$, that is, there are $(2 N+2)$-dimensional vectors $\mathbf{m}_{i}$ for $i=$ $0, \ldots, 2 N+1$ such that the left-hand side of (52) can be represented as

$$
\begin{aligned}
& \mathbf{m}_{i} \cdot\left(\frac{d l}{d t}, \frac{d \mathbf{h}}{d t}, \frac{d \mathbf{r}}{d t}\right) \\
& =\left\langle\left(\frac{\partial S}{\partial l}(z, 0, \mathbf{h}, \mathbf{r})-\frac{\partial}{\partial z} \Pi(\mathbf{h}) W\right) \frac{d l}{d t}\right. \\
& \left.+\left(\frac{\partial S}{\partial \mathbf{h}}(z, 0, \mathbf{h}, \mathbf{r})+\frac{\partial}{\partial \mathbf{h}} \Pi(\mathbf{h}) W\right) \frac{d \mathbf{h}}{d t}+\frac{\partial S}{\partial \mathbf{r}}(z, 0, \mathbf{h}, \mathbf{r}) \frac{d \mathbf{r}}{d t}, \Psi_{i}^{*}(\mathbf{h})\right\rangle,
\end{aligned}
$$




$$
\begin{aligned}
& \mathbf{m}_{N+1+i} \cdot\left(\frac{d l}{d t}, \frac{d \mathbf{h}}{d t}, \frac{d \mathbf{r}}{d t}\right) \\
& =\left\langle\left(\frac{\partial S}{\partial l}(z, 0, \mathbf{h}, \mathbf{r})-\frac{\partial}{\partial z} \Pi(\mathbf{h}) W\right) \frac{d l}{d t}\right. \\
& \left.+\left(\frac{\partial S}{\partial \mathbf{h}}(z, 0, \mathbf{h}, \mathbf{r})+\frac{\partial}{\partial \mathbf{h}} \Pi(\mathbf{h}) W\right) \frac{d \mathbf{h}}{d t}+\frac{\partial S}{\partial \mathbf{r}}(z, 0, \mathbf{h}, \mathbf{r}) \frac{d \mathbf{r}}{d t}, \Phi_{i}^{*}(\mathbf{h})\right\rangle
\end{aligned}
$$

for $i=0, \ldots, N$, where "." denotes the inner product in $\mathbb{R}^{2 N+2}$. The vector $\mathbf{m}_{i}=$ $\mathbf{m}_{i}(\mathbf{h}, \mathbf{r}, W)$ are defined in $\mathbf{h} \in H\left(h^{*}\right), \mathbf{r} \in R\left({\underset{\sim}{*}}^{*}\right)$, and $W \in X^{\omega}$ with $3 / 4<\omega<1$. Here we define a $(2 N+2) \times(2 N+2)$ matrix $\tilde{M}$ such that the $i$-th row corresponds to $\mathbf{m}_{i-1}$. By Lemma 3 and the same argument to the proof of Lemma $18, \tilde{M}=$ $\tilde{M}(\mathbf{h}, \mathbf{r}, W)$ can be estimated as shown in the following lemma.

Lemma 19 Set $\omega \in(3 / 4,1)$. There is a constant $C>0$ independent of $h^{*}, r^{*}$ such that if $h^{*}$ is sufficiently large and $r^{*}$ is sufficiently small, then

$$
\left\|\tilde{M}(\mathbf{h}, \mathbf{r}, W)-\left(\begin{array}{cc}
-M & 0 \\
0 & I_{N+1}
\end{array}\right)\right\| \leq C\left(\varepsilon^{1 / 4}+r^{*}+\|W\|_{\omega}\right)
$$

uniformly in $\mathbf{h} \in H\left(h^{*}\right), \mathbf{r} \in R\left(r^{*}\right)$, and $W \in X^{\omega}$, where $M$ is the same matrix as in the proof of Lemma 18, and

$$
\left\|\tilde{M}\left(\mathbf{h}_{1}, \mathbf{r}_{1}, W_{1}\right)-\tilde{M}\left(\mathbf{h}_{2}, \mathbf{r}_{2}, W_{2}\right)\right\| \leq C\left(\left|\mathbf{h}_{1}-\mathbf{h}_{2}\right|^{1 / 2}+\left|\mathbf{r}_{1}-\mathbf{r}_{2}\right|+\left\|W_{1}-W_{2}\right\|_{\omega}\right)
$$

uniformly in $\mathbf{h}_{1}, \mathbf{h}_{2} \in H\left(h^{*}\right), \mathbf{r}_{1}, \mathbf{r}_{2} \in R\left(r^{*}\right)$, and $W_{1}, W_{2} \in X^{\omega}$ with $\left\|W_{1}\right\|_{\omega} \leq 1$ and $\left\|W_{2}\right\|_{\omega} \leq 1$.

Proof Thanks to $\Pi(\mathbf{h}) W \in X^{\omega}$ and Lemma 3, we estimate

$$
\left|\left\langle\frac{\partial}{\partial z} \Pi(\mathbf{h}) W, \Psi_{i}^{*}(\mathbf{h})\right\rangle\right| \leq\|\Pi(\mathbf{h}) W\|_{\mathbb{R}^{2 N+2} \times L^{2}(\mathbb{T})}\left\|\Psi_{i}^{*}(\mathbf{h})\right\|_{X^{1}} \leq C\|W\|_{\omega}
$$

uniformly in $i=0, \ldots, N, \mathbf{h} \in H\left(h^{*}\right)$ and $W \in X^{\omega}$. From Lemma 5 , we have

$$
\begin{aligned}
& \left|\left\langle\frac{\partial}{\partial z} \Pi\left(\mathbf{h}_{1}\right) W_{1}, \Psi_{i}^{*}\left(\mathbf{h}_{1}\right)\right\rangle-\left\langle\frac{\partial}{\partial z} \Pi\left(\mathbf{h}_{2}\right) W_{2}, \Psi_{i}^{*}\left(\mathbf{h}_{2}\right)\right\rangle\right| \\
& \leq\left\|\Pi\left(\mathbf{h}_{1}\right) W_{1}-\Pi\left(\mathbf{h}_{2}\right) W_{2}\right\|_{\mathbb{R}^{2 N+2} \times L^{2}(\mathbb{T})}\left\|\Psi_{i}^{*}\left(\mathbf{h}_{1}\right)\right\|_{X^{1}} \\
& +\left\|\Pi\left(\mathbf{h}_{2}\right) W_{2}\right\|_{\mathbb{R}^{2 N+2} \times L^{2}(\mathbb{T})}\left\|\Psi_{i}^{*}\left(\mathbf{h}_{1}\right)-\Psi_{i}^{*}\left(\mathbf{h}_{2}\right)\right\|_{X^{1}} \\
& \leq C\left(\left|\mathbf{h}_{1}-\mathbf{h}_{2}\right|^{1 / 2}+\left\|W_{1}-W_{2}\right\|_{\omega}\right)
\end{aligned}
$$

uniformly in $i=0, \ldots, N, \mathbf{h}_{1}, \mathbf{h}_{2} \in H\left(h^{*}\right)$ and $W_{1}, W_{2} \in X^{\omega}$ with $\left\|W_{1}\right\|_{\omega} \leq 1$ and $\left\|W_{2}\right\|_{\omega} \leq 1$

From Lemma 5, we have

$$
\left|\left\langle\frac{\partial}{\partial h_{k}} \Pi(\mathbf{h}) W, \Psi_{i}^{*}(\mathbf{h})\right\rangle\right| \leq\left\|\frac{\partial}{\partial h_{k}} \Pi(\mathbf{h}) W\right\|_{X}\left\|\Psi_{i}^{*}(\mathbf{h})\right\|_{X^{1}} \leq C\|W\|_{\omega}
$$

uniformly in $i=0, \ldots, N, k=0, \ldots, N-1, \mathbf{h} \in H\left(h^{*}\right)$ and $W \in X^{\omega}$. In addition, we have

$$
\begin{aligned}
& \left|\left\langle\frac{\partial}{\partial h_{k}} \Pi\left(\mathbf{h}_{1}\right) W_{1}, \Psi_{i}^{*}\left(\mathbf{h}_{1}\right)\right\rangle-\left\langle\frac{\partial}{\partial h_{k}} \Pi\left(\mathbf{h}_{2}\right) W_{2}, \Psi_{i}^{*}\left(\mathbf{h}_{2}\right)\right\rangle\right| \\
& \leq C\left(\left|\mathbf{h}_{1}-\mathbf{h}_{2}\right|^{1 / 2}+\left\|W_{1}-W_{2}\right\|_{\omega}\right)
\end{aligned}
$$


uniformly in $i=0, \ldots, N, k=0, \ldots, N-1, \mathbf{h}_{1}, \mathbf{h}_{2} \in H\left(h^{*}\right)$ and $W_{1}, W_{2} \in X^{\omega}$ with $\left\|W_{1}\right\|_{\omega} \leq 1$ and $\left\|W_{2}\right\|_{\omega} \leq 1$.

From the inequalities above and by the same argument as in the proof of Lemma 18, we easily complete the proof of Lemma 19.

Next we study the right-hand side of (52). We set

$$
\begin{aligned}
F_{j}(\mathbf{h}, \mathbf{r}, W) & \equiv\left\langle\mathcal{L}(S(z, 0, \mathbf{h}, \mathbf{r})+\Pi(\mathbf{h}) W, \kappa), \Psi_{j}^{*}(\mathbf{h})\right\rangle, \\
F_{N+1+j}(\mathbf{h}, \mathbf{r}, W) & \equiv\left\langle\mathcal{L}(S(z, 0, \mathbf{h}, \mathbf{r})+\Pi(\mathbf{h}) W, \kappa), \Phi_{j}^{*}(\mathbf{h})\right\rangle
\end{aligned}
$$

for $j=0, \ldots, N$ and obtain the lowest order terms of $F_{j}(\mathbf{h}, \mathbf{r}, W)$.

Lemma 20 Assume $\omega \in(3 / 4,1)$ and let $\zeta$ be a positive constant satisfying (15). There is a constant $C>0$ independent of $h^{*}, r^{*}, \kappa$ such that if $h^{*}$ is sufficiently large and $r^{*},|\kappa|$ are sufficiently small, then

$$
\begin{aligned}
& \left|F_{j}(\mathbf{h}, \mathbf{r}, W)+r_{j}\right| \leq C\left(\varepsilon^{1 / 2}+\left(r^{*}\right)^{2}+|\kappa|+\|W\|_{\omega}^{\zeta+1}\right), \\
& \left|F_{N+1+j}(\mathbf{h}, \mathbf{r}, W)\right| \leq C\left(\varepsilon^{1 / 2}+\left(r^{*}\right)^{2}+|\kappa|+\|W\|_{\omega}^{\zeta+1}\right)
\end{aligned}
$$

uniformly in $j=0, \ldots, N, \mathbf{h} \in H\left(h^{*}\right), \mathbf{r} \in R\left(r^{*}\right), W \in X^{\omega} \cap E^{\perp}\left(\mathbf{h}^{*}\right)$ with $\|W\|_{\omega} \leq 1$, and

$$
\left|F_{j}\left(\mathbf{h}_{1}, \mathbf{r}_{1}, W_{1}\right)-F_{j}\left(\mathbf{h}_{2}, \mathbf{r}_{2}, W_{2}\right)\right| \leq C\left(\left|\mathbf{h}_{1}-\mathbf{h}_{2}\right|^{\zeta}+\left|\mathbf{r}_{1}-\mathbf{r}_{2}\right|+\left\|W_{1}-W_{2}\right\| \|_{\omega}^{\zeta}\right)
$$

uniformly in $j=0, \ldots, 2 N+1, \mathbf{h}_{1}, \mathbf{h}_{2} \in H\left(h^{*}\right), \mathbf{r}_{1}, \mathbf{r}_{2} \in R\left(r^{*}\right), W_{1}, W_{2} \in X^{\omega} \cap$ $E^{\perp}\left(\mathbf{h}^{*}\right)$ with $\left\|W_{1}\right\|_{\omega} \leq 1$ and $\left\|W_{2}\right\|_{\omega} \leq 1$.

Proof First of all, we compute $\mathcal{L}(S(z, 0, \mathbf{h}, \mathbf{r})+\Pi(\mathbf{h}) W, \kappa)$ under the assumption of $W \in X^{1} \cap E^{\perp}\left(\mathbf{h}^{*}\right)$. Set $\Pi(\mathbf{h}) W \equiv{ }^{t}\left(w_{0}^{z}, w_{0}^{y}, \ldots, w_{N}^{z}, w_{N}^{y}, w\right)$ and denote the $(2 N+3)$-th component of $S(z, 0, \mathbf{h}, \mathbf{r})+\Pi(\mathbf{h}) W$ by a function $u$. Note that the positive constant $\zeta$ is less than $1 / 2$ because of (15).

We easily see $\mathcal{L}_{i}^{z}(S(z, 0, \mathbf{h}, \mathbf{r})+\Pi(\mathbf{h}) W)=r_{i}+w_{i}^{y}$ and

$$
\begin{aligned}
\mathcal{L}_{i}^{y}\left(S(z, 0, \mathbf{h}, \mathbf{r})+\Pi(\mathbf{h}) W, \kappa_{1}\right)= & \left.-\mu_{0} w_{i}^{y}+\gamma^{\prime}(p(\rho))\left(p^{\prime}(\rho) w_{i}^{z}+w\left(\bar{z}_{i}+\rho\right)\right)\right) \\
& \left.-\gamma^{\prime}(p(-\rho))\left(p^{\prime}(-\rho) w_{i}^{z}+w\left(\bar{z}_{i}-\rho\right)\right)\right) \\
& +R_{+}-R_{-}-\kappa_{1}\left(r_{i}+w_{i}^{y}-c_{0}\right)
\end{aligned}
$$

where

$$
\begin{aligned}
R_{ \pm} & \equiv \gamma\left(u\left(\bar{z}_{i}+w_{i}^{z} \pm \rho\right)\right) \\
& -\left(\gamma(p( \pm \rho))+\gamma^{\prime}(p( \pm \rho))\left(p^{\prime}( \pm \rho) w_{i}^{z}+r_{i} \psi( \pm \rho)+w\left(\bar{z}_{i} \pm \rho\right)\right)\right) .
\end{aligned}
$$

Since $p$ and $\psi$ decay exponentially as $|z| \rightarrow \infty$, we have

$$
\left|R_{ \pm}\right| \leq C\left(\varepsilon+\left(r^{*}\right)^{2}+\|W\|_{\omega}^{\zeta+1}\right)
$$

uniformly in $i=0, \ldots, N, \mathbf{h} \in H\left(h^{*}\right), \mathbf{r} \in R\left(r^{*}\right)$, and $W \in X^{\omega}$. 
Next we study $\mathcal{L}^{u}\left(S(z, 0, \mathbf{h}, \mathbf{r})+\Pi(\mathbf{h}) W, \kappa_{2}\right)$. Direct calculations give us

$$
\begin{aligned}
& \mathcal{L}^{u}\left(S(z, 0, \mathbf{h}, \mathbf{r})+\Pi(\mathbf{h}) W, \kappa_{2}\right) \\
& =\sum_{k=0}^{N}\left(\bar{p}^{\prime \prime}\left(z-\bar{z}_{k}\right)-c_{0} \bar{p}^{\prime}\left(z-\bar{z}_{k}\right)-\alpha \bar{p}\left(z-\bar{z}_{k}\right)+\bar{f}\left(z-\bar{z}_{k}, s_{0}\right)\right) \\
& +\sum_{k=0}^{N} r_{k}\left(\bar{\psi}^{\prime \prime}\left(z-\bar{z}_{k}\right)-c_{0} \bar{\psi}^{\prime}\left(z-\bar{z}_{k}\right)-\alpha \bar{\psi}\left(z-\bar{z}_{k}\right)+\bar{\phi}\left(z-\bar{z}_{k}\right)\right) \\
& +\sum_{k=0}^{N}\left(\bar{f}\left(z-\bar{z}_{k}-w_{k}^{z}, s_{0}+\kappa_{2}\right)-\bar{f}\left(z-\bar{z}_{k}, s_{0}\right)\right) \\
& +\sum_{k=0}^{N} w_{k}^{z}\left(s_{0} \delta_{\bar{z}_{k}-\rho}-\left(s_{0}-1\right) \delta_{\bar{z}_{k}}-\delta_{\bar{z}_{k}+\rho}\right)-\sum_{k=0}^{N} r_{k} \bar{\phi}\left(z-\bar{z}_{k}\right) \\
& +\frac{\partial^{2} w}{\partial z^{2}}-c_{0} \frac{\partial w}{\partial z}-\alpha w-\sum_{k=0}^{N} w_{k}^{z}\left(s_{0} \delta_{\bar{z}_{k}-\rho}-\left(s_{0}-1\right) \delta_{\bar{z}_{k}}-\delta_{\bar{z}_{k}+\rho}\right) .
\end{aligned}
$$

Putting $\varphi=\overline{\phi^{*}}$ and $\overline{\psi^{*}}$, we see that

$$
\begin{aligned}
& \left|\left\langle\bar{p}^{\prime \prime}\left(\cdot-\bar{z}_{k}\right)-c_{0} \bar{p}^{\prime}\left(\cdot-\bar{z}_{k}\right)-\alpha \bar{p}\left(\cdot-\bar{z}_{k}\right)+\bar{f}\left(\cdot-\bar{z}_{k}, s_{0}\right), \varphi\left(\cdot-\bar{z}_{j}\right)\right\rangle_{H^{1}(\mathbb{T})}\right| \leq C \varepsilon^{1 / 2}, \\
& \left|\left\langle\bar{\psi}^{\prime \prime}\left(\cdot-\bar{z}_{k}\right)-c_{0} \bar{\psi}^{\prime}\left(\cdot-\bar{z}_{k}\right)-\alpha \bar{\psi}\left(\cdot-\bar{z}_{k}\right)+\bar{\phi}\left(\cdot-\bar{z}_{k}\right), \varphi\left(\cdot-\bar{z}_{j}\right)\right\rangle_{H^{1}(\mathbb{T})}\right| \leq C \varepsilon^{1 / 2}
\end{aligned}
$$

uniformly in $j, k=0, \ldots, N$ and $\mathbf{h} \in H\left(h^{*}\right)$. In addition,

$$
\begin{aligned}
& \mid\left\langle\bar{f}\left(z-\bar{z}_{k}-w_{k}^{z}, s_{0}+\kappa_{2}\right)-\bar{f}\left(z-\bar{z}_{k}, s_{0}\right)\right) \\
& \left.\quad+w_{k}^{z}\left(s_{0} \delta_{\bar{z}_{k}-\rho}-\left(s_{0}-1\right) \delta_{\bar{z}_{k}}-\delta_{\bar{z}_{k}+\rho}\right), \varphi\left(\cdot-\bar{z}_{j}\right)\right\rangle_{H^{1}(\mathbb{T})} \mid \leq C\left(\varepsilon+|\kappa|+\|W\|_{\omega}^{3 / 2}\right)
\end{aligned}
$$

uniformly in $j, k=0, \ldots, N, \mathbf{h} \in H\left(h^{*}\right)$, and $W \in X^{\omega}$ with $\|W\|_{\omega} \leq 1$.

Due to $L(\mathbf{h})^{*} \Phi_{j}^{*}(\mathbf{h}), L(\mathbf{h})^{*} \Psi_{j}^{*}(\mathbf{h}) \in E(\mathbf{h})^{*}$, we see

$$
\left\langle L(\mathbf{h}) \Pi(\mathbf{h}) W, \Phi_{j}^{*}(\mathbf{h})\right\rangle=\left\langle L(\mathbf{h}) \Pi(\mathbf{h}) W, \Psi_{j}^{*}(\mathbf{h})\right\rangle=0
$$

in any $j=0, \ldots, N$. Thanks to (32) and Lemma 9, we have

$$
\left\langle\hat{\Phi}_{k}(\mathbf{h}), \Phi_{j}^{*}(\mathbf{h})\right\rangle=\left\langle\hat{\Phi}_{k}(\mathbf{h}), \Psi_{j}^{*}(\mathbf{h})\right\rangle-\delta_{k j}=0
$$

in any $j, k=0, \ldots, N$. Therefore we have (54) for any $W \in X^{1} \cap E^{\perp}\left(\mathbf{h}^{*}\right)$. Then, a usual density argument implies that (54) holds true in $W \in X^{\omega} \cap E^{\perp}\left(\mathbf{h}^{*}\right)$.

The inequality (55) can be proved by the same arguments above and owing to Lemmas 3, 5, 10.

From Lemma $19, \tilde{M}$ is invertible. We set $\mathbf{V} \equiv(\mathbf{h}, \mathbf{r}, W)$ and define $\mathbf{G}(\mathbf{V})=$ ${ }^{t}\left(G_{0}, \ldots, G_{2 N+1}\right)(\mathbf{V}) \equiv\left(\tilde{M}(\mathbf{V})^{-1}\right)^{t}\left(F_{0}, \ldots, F_{2 N+1}\right)(\mathbf{V})$. Then $(l, \mathbf{h}, \mathbf{r})$ is governed by reduced equations

$$
\begin{cases}\frac{d l}{d t}=G_{0}(\mathbf{V}), & \\ \frac{d h_{i}}{d t}=G_{i+1}(\mathbf{V}), & (i=0, \ldots, N-1), \\ \frac{d r_{i}}{d t}=G_{N+1+i}(\mathbf{V}), & (i=0, \ldots, N) .\end{cases}
$$


Substitution of (56) into the left-hand side of (53) results in

$$
\frac{\partial W}{\partial t}=\tilde{L}(\mathbf{h}) W+\tilde{G}(\mathbf{V})
$$

where

$$
\begin{aligned}
\tilde{G}(\mathbf{V}) \equiv \Pi(\mathbf{h})^{-1} R(\mathbf{h}) \\
\quad\left[\mathcal{L}(S(z, 0, \mathbf{h}, \mathbf{r}), 0)+L_{2}(\mathbf{V})+\left(\frac{\partial S}{\partial l}(z, 0, \mathbf{h}, \mathbf{r})-\frac{\partial}{\partial z} \Pi(\mathbf{h}) W\right) G_{0}(\mathbf{V})\right. \\
\left.-\left(\frac{\partial S}{\partial \mathbf{h}}(z, 0, \mathbf{h}, \mathbf{r})+\frac{\partial}{\partial \mathbf{h}} \Pi(\mathbf{h}) W\right) \mathbf{G}_{1}(\mathbf{V})-\left(\frac{\partial S}{\partial \mathbf{r}}(z, 0, \mathbf{h}, \mathbf{r})\right) \mathbf{G}_{2}(\mathbf{V})\right], \\
L_{2}(\mathbf{V}) \equiv \mathcal{L}(S(z, 0, \mathbf{h}, \mathbf{r})+\Pi(\mathbf{h}) W, \kappa)-\mathcal{L}(S(z, 0, \mathbf{h}, \mathbf{r}), 0)-L(\mathbf{h}) \Pi(\mathbf{h}) W \\
\mathbf{G}_{1}(\mathbf{V}) \equiv{ }^{t}\left(G_{1}, \ldots, G_{N}\right)(\mathbf{V}), \quad \mathbf{G}_{2}(\mathbf{V}) \equiv{ }^{t}\left(G_{N+1}, \ldots, G_{2 N+1}\right)(\mathbf{V}) .
\end{aligned}
$$

In the end, we have the following lemma. It can be proved by the same way as in the proofs of Lemmas 19 and 20 so that we omit the details of the proof. Here we note that the matrix $M$ defined in the proof of Lemma 18 has the inverse matrix $M^{-1}$, which is a tridiagonal matrix and represented by $M^{-1}=\left(\hat{m}_{i k}\right)_{i, k}$, where $\hat{m}_{i k}(i, k=1, \ldots, N+1)$ is given by

$$
\hat{m}_{i k} \equiv \begin{cases}1, & i=k, \\ -1, & i=k+1, \\ 0, & \text { otherwise. }\end{cases}
$$

Lemma 21 Assume $\omega \in(3 / 4,1)$ and let $\zeta$ be a positive constant satisfying (15). There is a constant $C>0$ independent of $h^{*}, r^{*}, \kappa$ such that if $h^{*}$ is sufficiently large and $r^{*},|\kappa|$ are sufficiently small, then

$$
\begin{aligned}
\left|G_{0}(\mathbf{V})-r_{0}\right| & \leq C\left(\varepsilon^{1 / 2}+\left(r^{*}\right)^{2}+|\kappa|+\|W\|_{\omega}^{\zeta+1}\right), \\
\left|G_{i+1}(\mathbf{V})-\left(r_{i+1}-r_{i}\right)\right| & \leq C\left(\varepsilon^{1 / 2}+\left(r^{*}\right)^{2}+|\kappa|+\|W\|_{\omega}^{\zeta+1}\right), \\
\left|G_{N+j+1}(\mathbf{V})\right| & \leq C\left(\varepsilon^{1 / 2}+\left(r^{*}\right)^{2}+|\kappa|+\|W\|_{\omega}^{\zeta+1}\right), \\
\|\tilde{G}(\mathbf{V})\|_{X} & \leq C\left(\varepsilon^{1 / 2}+\left(r^{*}\right)^{2}+|\kappa|+\|W\|_{\omega}^{\zeta+1}\right)
\end{aligned}
$$

uniformly in $i=0, \ldots, N-1, j=0, \ldots, N, \mathbf{V}=(\mathbf{h}, \mathbf{r}, W) \in H\left(h^{*}\right) \times R\left(r^{*}\right) \times$ $\left(X^{\omega} \cap E^{\perp}\left(\mathbf{h}^{*}\right)\right)$ with $\|W\|_{\omega} \leq 1$, where $\mathbf{r}={ }^{t}\left(r_{0}, \ldots, r_{N}\right)$. In addition, one has

$$
\begin{array}{r}
\left|G_{i}\left(\mathbf{V}_{1}\right)-G_{i}\left(\mathbf{V}_{2}\right)\right| \leq C\left(\left|\mathbf{h}_{1}-\mathbf{h}_{2}\right|^{\zeta}+\left|\mathbf{r}_{1}-\mathbf{r}_{2}\right|+\left\|W_{1}-W_{2}\right\|_{\omega}^{\zeta}\right), \\
\left\|\tilde{G}\left(\mathbf{V}_{1}\right)-\tilde{G}\left(\mathbf{V}_{2}\right)\right\|_{X} \leq C\left(\left|\mathbf{h}_{1}-\mathbf{h}_{2}\right|^{\zeta}+\left|\mathbf{r}_{1}-\mathbf{r}_{2}\right|+\left\|W_{1}-W_{2}\right\|_{\omega}^{\zeta}\right)
\end{array}
$$

uniformly in $i=0, \ldots, 2 N+1, \mathbf{V}_{1}=\left(\mathbf{h}_{1}, \mathbf{r}_{1}, W_{1}\right), \mathbf{V}_{2}=\left(\mathbf{h}_{2}, \mathbf{r}_{2}, W_{2}\right) \in H\left(h^{*}\right) \times$ $R\left(r^{*}\right) \times\left(X^{\omega} \cap E^{\perp}\left(\mathbf{h}^{*}\right)\right)$ with $\left\|W_{1}\right\|_{\omega} \leq 1$ and $\left\|W_{2}\right\|_{\omega} \leq 1$.

Eventually we derive a decomposed system from (4) as

$$
\left\{\begin{array}{l}
\frac{d \mathbf{h}}{d t}=\mathbf{G}_{1}(\mathbf{h}, \mathbf{r}, W), \\
\frac{d \mathbf{r}}{d t}=\mathbf{G}_{2}(\mathbf{h}, \mathbf{r}, W), \\
\frac{\partial W}{\partial t}=\tilde{L}(\mathbf{h}) W+\tilde{G}(\mathbf{h}, \mathbf{r}, W) .
\end{array}\right.
$$


Note that the variable $l$ can be determined by the first differential equation (56). By solving the third equation of (60) and estimating the function $W$, we will obtain our main result.

Before we state our main theorem, we give a few remarks on the initial value $U_{0}$ of (4) at $t=0$. As stated in Section $1, U_{0}$ is supposed to be close to $S\left(z, l_{0}, \mathbf{h}_{0}, \mathbf{r}_{0}\right)$ for some $l_{0} \in \mathbb{R}, \mathbf{h}_{0} \in H\left(h^{*}\right), \mathbf{r}_{0} \in R\left(r^{*}\right)$. More precisely, we fix constants $C_{*}, C_{1}>$ 0 and $\omega \in(3 / 4,1)$ independent of $h^{*}, r^{*}, \kappa$, and assume that $U_{0}$ satisfies

$$
U_{0} \in X^{\omega}, \quad\left\|U_{0}-S\left(\cdot, l_{0}, \mathbf{h}_{0}, \mathbf{r}_{0}\right)\right\|_{\omega} \leq \eta \equiv C_{1}\left(\varepsilon^{1 / 2}+\left(r^{*}\right)^{2}+|\kappa|\right) .
$$

Then it follows from Lemma 18 that if $L, h^{*}$ are sufficiently large with (6) for $C_{*}$ and $r^{*},|\kappa|$ are sufficiently small, there are $l \in \mathbb{R}, \mathbf{h} \in H\left(\tilde{h}^{*}\right), \mathbf{r} \in R\left(\tilde{r}^{*}\right)$ and a constant $C>0$ independent of $h^{*}, r^{*}, \kappa$ such that $U_{0}-S(\cdot, l, \mathbf{h}, \mathbf{r}) \in X^{\omega} \cap E^{\perp}(\mathbf{h})$ and $\left\|U_{0}-S(\cdot, l, \mathbf{h}, \mathbf{r})\right\|_{\omega} \leq C \eta$, where $\tilde{h}^{*} \equiv h^{*}-C \eta, \tilde{\varepsilon} \equiv e^{-\beta_{1} \tilde{h}^{*}}$, and $\tilde{r}^{*} \equiv r^{*}+C \eta$. Since $\eta$ is sufficiently small, $\tilde{h}^{*}$ is sufficiently large with (6) for $L, \tilde{r}^{*}$ is sufficiently small, and a constant $\tilde{C}_{*}$ close to $C_{*}$ and independent of $h^{*}, r^{*}, \kappa$. Moreover, we easily see that $\varepsilon<\tilde{\varepsilon}$ and $r^{*}<\tilde{r}^{*}$. Thus we replace $\left(\tilde{h}^{*}, \tilde{r}^{*}, \tilde{\varepsilon}, \tilde{C}^{*}\right) \rightarrow\left(h^{*}, r^{*}, \varepsilon, C^{*}\right)$ and $(l, \mathbf{h}, \mathbf{r}) \rightarrow\left(l_{0}, \mathbf{h}_{0}, \mathbf{r}_{0}\right)$, and assume instead of (61) that there is a constant $C>$ 0 independent of $h^{*}, r^{*}, \kappa$ such that $U_{0}$ satisfies $U_{0}-S\left(\cdot, l_{0}, \mathbf{h}_{0}, \mathbf{r}_{0}\right) \in X^{\omega} \cap E^{\perp}\left(\mathbf{h}_{0}\right)$ and $\left\|U_{0}-S\left(\cdot, l_{0}, \mathbf{h}_{0}, \mathbf{r}_{0}\right)\right\|_{\omega} \leq C\left(\varepsilon^{1 / 2}+\left(r^{*}\right)^{2}+|\kappa|\right)$ for some $l_{0} \in \mathbb{R}, \mathbf{h}_{0} \in H\left(h^{*}\right), \mathbf{r}_{0} \in$ $R\left(r^{*}\right)$.

Now we are in a position to state and prove our main theorem.

Theorem 1 Put $N \geq 1$ and $\omega \in(3 / 4,1)$. Let $C_{*}, C_{1}>0$ be arbitrary fixed constants. Assume (A1)-(A3), and suppose that $L, h^{*}$ are sufficiently large with (6) for $C_{*}$ and $r^{*},|\kappa|$ are sufficiently small. Let $U_{0}=U_{0}(z)$ be a function such that $U_{0}-S\left(\cdot, l_{0}, \mathbf{h}_{0}, \mathbf{r}_{0}\right) \in X^{\omega} \cap E^{\perp}\left(\mathbf{h}_{0}\right)$ and $\left\|U_{0}-S\left(\cdot, l_{0}, \mathbf{h}_{0}, \mathbf{r}_{0}\right)\right\|_{\omega} \leq C_{1}\left(\varepsilon^{1 / 2}+\left(r^{*}\right)^{2}+\right.$ $|\kappa|)$ for some $l_{0} \in \mathbb{R}, \mathbf{h}_{0} \in H\left(h^{*}\right), \mathbf{r}_{0} \in R\left(r^{*}\right)$. Then, there exist a positive constant $C_{2}>0$ independent of $h^{*}, r^{*}, \kappa$ and $l(t) \in \mathbb{R}, \mathbf{h}(t) \in H\left(h^{*}\right), \mathbf{r}(t) \in R\left(r^{*}\right)$ such that $(l(0), \mathbf{h}(0), \mathbf{r}(0))=\left(l_{0}, \mathbf{h}_{0}, \mathbf{r}_{0}\right)$, and $U(\cdot, t)-S(\cdot, l(t), \mathbf{h}(t), \mathbf{r}(t)) \in X^{\omega} \cap E^{\perp}(\mathbf{h}(t))$ and

$$
\|U(\cdot, t)-S(\cdot, l(t), \mathbf{h}(t), \mathbf{r}(t))\|_{\omega} \leq C_{2}\left(\varepsilon^{1 / 2}+\left(r^{*}\right)^{2}+|\kappa|\right)
$$

hold true in $t>0$ as long as $\mathbf{h}(t) \in H\left(h^{*}\right), \mathbf{r}(t) \in R\left(r^{*}\right)$, where $U(z, t)$ is a solution of (4) with $U(z, 0)=U_{0}(z)$. In addition, there exists a positive constant $C_{3}$ independent of $h^{*}, r^{*}, \kappa$ such that

$$
\left|\frac{d l}{d t}-r_{0}\right|+\left|\frac{d h_{i}}{d t}-\left(r_{i+1}-r_{i}\right)\right|+\left|\frac{d r_{i}}{d t}\right| \leq C_{3}\left(\varepsilon^{1 / 2}+\left(r^{*}\right)^{2}+|\kappa|\right)
$$

for $i=0, \ldots, N$, where $h_{i}=h_{i}(t)$ and $r_{i}=r_{i}(t)$ are the $i$-th components of $\mathbf{h}(t)$ and $\mathbf{r}(t)$, respectively, and $h_{N}=L-\sum_{i=0}^{N-1} h_{i}$ and $r_{N+1}=r_{0}$.

Proof Throughout this proof, we denote general positive constants by $C$ independent of $h^{*}, r^{*}, \kappa, t$. Corollary 1 after Lemma 18 implies that there exist $t_{0}>0$ and $(l, \mathbf{h}, \mathbf{r}, W)=(l(t), \mathbf{h}(t), \mathbf{r}(t), W(z, t)) \in \mathbb{R} \times H\left(h^{*}\right) \times R\left(r^{*}\right) \times\left(X^{\omega} \cap E^{\perp}\left(\mathbf{h}^{*}\right)\right)$ such that the solution $U(z, t)$ can be decomposed by $(l, \mathbf{h}, \mathbf{r}, W)$ as in $(50)$ in $t \in\left[0, t_{0}\right]$. Moreover, $(l, \mathbf{h}, \mathbf{r}, W)$ is differentiable with respect to $t \in\left(0, t_{0}\right)$ in $\mathbb{R}^{2 N+2} \times X^{\omega}$. Then, $(\mathbf{h}, \mathbf{r}, W)$ is a solution of $(60)$ in $t \in\left(0, t_{0}\right)$ with the initial value $\left(\mathbf{h}_{0}, \mathbf{r}_{0}, W\right)$ at $t=0$. 
Hereafter we omit the $z$-dependency of $W(z, t)$ and abbreviate $W(z, t)$ as $W(t)$. From (60), we have

$$
W(t)=T(t, 0 ; \mathbf{h}) W_{0}+\int_{0}^{t} T(t, a ; \mathbf{h}) \tilde{G}(\mathbf{h}(a), \mathbf{r}(a), W(a)) d a .
$$

From the assumption and Lemma $12,\left\|W_{0}\right\|_{\omega} \leq C\left(\varepsilon^{1 / 2}+\left(r^{*}\right)^{2}+|\kappa|\right)$. Then it follows from Lemma 21 that

$$
\begin{aligned}
\|W(t)\|_{\omega} & \leq C e^{-\beta_{2} t}\left\|W_{0}\right\|_{\omega} \\
& +C \int_{0}^{t} \max \left\{1,(t-a)^{-\omega}\right\} e^{-\beta_{2}(t-a)}\|\tilde{G}(\mathbf{h}(a), \mathbf{r}(a), W(a))\|_{X} d a \\
& \leq C\left(\varepsilon^{1 / 2}+\left(r^{*}\right)^{2}+|\kappa|\right)+C \max _{t \in\left[0, t_{0}\right]}\|W(t)\|_{\omega}^{\zeta+1},
\end{aligned}
$$

where $\zeta$ is a positive constant satisfying (15). If $h^{*}$ is sufficiently large and $r^{*},|\kappa|$ are sufficiently small, we have $\|W(t)\|_{\omega} \leq C\left(\varepsilon^{1 / 2}+\left(r^{*}\right)^{2}+|\kappa|\right)$ in $0 \leq t \leq t_{0}$ as far as $\mathbf{h}(t) \in H\left(h^{*}\right)$ and $\mathbf{r}(t) \in R\left(r^{*}\right)$.

In the case of $\mathbf{h}\left(t_{0}\right) \in H\left(h^{*}\right)$ and $\mathbf{r}\left(t_{0}\right) \in R\left(r^{*}\right)$, we can find $t_{1}>t_{0}$ and $\left(l_{1}, \mathbf{h}_{1}, \mathbf{r}_{1}, W_{1}\right)=\left(l_{1}(t), \mathbf{h}_{1}(t), \mathbf{r}_{1}(t), W_{1}(t)\right) \in \mathbb{R} \times H\left(h^{*}\right) \times R\left(r^{*}\right) \times\left(X^{\omega} \cap E^{\perp}\left(\mathbf{h}^{*}\right)\right)$ such that the solution $U(z, t)$ can be decomposed by $\left(l_{1}, \mathbf{h}_{1}, \mathbf{r}_{1}, W_{1}\right)$ as in (50) in $t \in\left[t_{0}, t_{1}\right]$. Moreover, $\left(l_{1}, \mathbf{h}_{1}, \mathbf{r}_{1}, W_{1}\right)$ is differentiable with respect to $t \in\left(t_{0}, t_{1}\right)$ in $\mathbb{R}^{2 N+2} \times X^{\omega}$, and then $\left(\mathbf{h}_{1}, \mathbf{r}_{1}, W_{1}\right)$ is a solution of $(60)$ in $t \in\left(t_{0}, t_{1}\right)$ with the initial value $\left(\mathbf{h}\left(t_{0}\right), \mathbf{r}\left(t_{0}\right), W\left(t_{0}\right)\right)$ at $t=t_{0}$. Since the solutions $(\mathbf{h}(t), \mathbf{r}(t), W(t))$ and $\left(\mathbf{h}_{1}(t), \mathbf{r}_{1}(t), W_{1}(t)\right)$ are continuous for $t \in\left[0, t_{0}\right]$ and $t \in\left[t_{0}, t_{1}\right]$ in $\mathbb{R}^{2 N+2} \times X^{\omega}$, respectively, we can also define all derivatives with respect to $t$ at $t=t_{0}$ and see

$$
\frac{d \mathbf{h}}{d t}\left(t_{0}\right)=\frac{d \mathbf{h}_{1}}{d t}\left(t_{0}\right), \quad \frac{d \mathbf{r}}{d t}\left(t_{0}\right)=\frac{d \mathbf{r}_{1}}{d t}\left(t_{0}\right), \quad \frac{\partial W}{\partial t}\left(t_{0}\right)=\frac{\partial W_{1}}{\partial t}\left(t_{0}\right) .
$$

Therefore we have constructed a solution of (60) in $t \in\left[0, t_{1}\right]$. By a similar argument, we can continue the solution as long as $\mathbf{h}(t) \in H\left(h^{*}\right), \mathbf{r}(t) \in R\left(r^{*}\right)$. This completes the proof.

Acknowledgements The authors would like to thank Nobuhiko J. Suematsu (Meiji University, Japan) and Kei Nishi (Hokkaido University, Japan) for stimulating discussions. We would like to express their appreciation to the referees for their useful suggestions and comments which have improved the original manuscript and made us to pay attention to a saddle-node bifurcation in (2). We would like to thank Editage (www.editage.jp) for English language editing. This work was funded by the Japan Society for the Promotion of Science (JSPS) through Grants-in-Aid for Young Scientists (B) (No.15K17594), Scientific Research (B) (Generative Research Fields) (No.26310212) and JST CREST Grant Number JPMJCR14D3, Japan.

\section{References}

1. G. Caginalp and P. C. Fife, Dynamics of layered interfaces arising from phase boundaries, SIAM Journal on Applied Mathematics, 48.3 (1988), pp.506-518.

2. M. K. Chaudhury and G. M. Whitesides, How to make water run uphill, Science, 256 (1992), pp.1539-1541.

3. S.-I. Ei, The motion of weakly interacting pulses in reaction-diffusion systems, J. Dynam. Differential Equations, 14 (2002), pp.85-137. 
4. S.-I. Ei, K. Ikeda, M. Nagayama and A. Tomoeda, Reduced model from a reaction-diffusion system of collective motion of camphor boats, Discrete Contin. Dyn. Syst. Ser. S, 8 (2015), pp.847-856.

5. S.-I. Ei, K. Ikeda, M. Nagayama, M. Okamoto and A. Tomoeda, Reduced model of a reaction-diffusion system for the collective motion of camphor boats, Physical Review E, 99 (2019), 062208.

6. S.-I. Ei, H. Kitahata, Y. Koyano and M. Nagayama, Interaction of non-radially symmetric camphor particles, Physica D: Nonlinear Phenomena, 366 (2018), pp.10-26.

7. S.-I. Ei, M. Mimura and M. Nagayama, Pulse-pulse interaction in reaction-diffusion systems, Physica D, 165 (2002), pp.176-198.

8. L. C. Evans, Partial differential equations, volume 19 of Graduate Studies in Mathematics. American Mathematical Society, Providence, RI, 2010, second edition.

9. E. Heisler, N. J. Suematsu, A. Awazu and H. Hiraku, Swarming of self-propelled camphor boats, Physical Review E, 85 (2012), 055201.

10. D. Helbing, I. Farkas and T. Vicsek, Simulating dynamical features of escape panic, Nature, 407 (2000), pp.487-490.

11. D. Henry, Geometric theory of semilinear parabolic equations, Lecture Notes in Math. 840, Springer, 1981

12. D. Hilhorst, Y. Nishiura and M. Mimura, A free boundary problem arising in some reacting-diffusing system, Proceedings of the Royal Society of Edinburgh Section A: Mathematics, 118 (1991), pp.355-378.

13. T. Ikeda, M. Nagayama and H. Ikeda, Bifurcation of a helical wave from a traveling wave, Japan journal of industrial and applied mathematics, 21.3 (2004), pp.405-424.

14. M. Inaba, H. Yamanaka and S. Kondo, Pigment pattern formation by contact-dependent depolarization, Science, 335 (2012), 677.

15. T. Kato, Perturbation theory for linear operators. Reprint of the corr. print. of the 2nd ed. 1980. Classics in Mathematics, Berlin: Springer-Verlag, 1995.

16. T. Miura and R. Tanaka, In Vitro vasculogenesis models revisited-measurement of VEGF diffusion in matrigel, Mathematical Modelling of Natural Phenomena, 4 (2009), pp.118-130.

17. M. Nagayama, S. Nakata, Y. Doi and Y. Hayashima, A theoretical and experimental study on the unidirectional motion of a camphor disk, Physica D: Nonlinear Phenomena, 194 (2004), pp.151-165.

18. S. Nakata, Y. Iguchi, S. Ose, M. Kuboyama, T. Ishii and K. Yoshikawa, Self-rotation of a camphor scraping on water: new insight into the old problem, Langmuir, 13 (1997), pp.4454-4458.

19. H. Ninomiya and C.-H. Wu, Traveling Curved Waves in Two-Dimensional Excitable Media, SIAM Journal on Mathematical Analysis, 49.2 (2017), pp.777-817.

20. N. J. Suematsu, S. Nakata, A. Awazu and H. Nishimori, Collective behavior of inanimate boats, Physical Review E, 81 (2010), 056210.

21. A. Tomoeda, K. Nishinari. D. Chowdhury and A. Schadschneider, An information-based traffic control in a public conveyance system: Reduced clustering and enhanced efficiency, Physica A: Statistical Mechanics and its Applications, 384 (2007), pp.600-612.

22. A. Tomoeda, D. Yanagisawa, T. Imamura and K. Nishinari, Propagation speed of a starting wave in a queue of pedestrians, Physical Review E, 86 (2012), 036113. 